\title{
Semiclassical Theory of the Structure of the Hydrogen Spectrum in Near-Perpendicular Electric and Magnetic fields: Derivations and Formulas for Einstein-Brillouin-Keller-Maslov Quantization and Description of Monodromy
}

Christopher Robert Schleif

William \& Mary

John B. Delos

William \&Mary, jbdelos@wm.edu

Follow this and additional works at: https://scholarworks.wm.edu/aspubs

Part of the Physics Commons

\section{Recommended Citation}

Schleif, Christopher Robert and Delos, John B., Semiclassical Theory of the Structure of the Hydrogen Spectrum in Near-Perpendicular Electric and Magnetic fields: Derivations and Formulas for EinsteinBrillouin-Keller-Maslov Quantization and Description of Monodromy (2008). Physical Review A, 77(4). https://doi.org/10.1103/PhysRevA.77.043422

This Article is brought to you for free and open access by the Arts and Sciences at W\&M ScholarWorks. It has been accepted for inclusion in Arts \& Sciences Articles by an authorized administrator of W\&M ScholarWorks. For more information, please contact scholarworks@wm.edu. 


\title{
Semiclassical theory of the structure of the hydrogen spectrum in near-perpendicular electric and magnetic fields: Derivations and formulas for Einstein-Brillouin-Keller-Maslov quantization and description of monodromy
}

\author{
Christopher R. Schleif* and John B. Delos ${ }^{\dagger}$ \\ The College of William and Mary, Williamsburg, Virginia 23187-8795, USA
}

(Received 24 January 2008; published 29 April 2008)

\begin{abstract}
In a previous paper [Schleif and Delos, Phys. Rev. A 76, 013404 (2007)] we described the spectrum of hydrogen atoms in near-perpendicular electric and magnetic fields. We displayed a number of previously unrecognized structures in the spectrum, most of which are connected with a classical phenomenon called "nontrivial monodromy of action and angle variables in a Hamiltonian system," or simply "monodromy." In that paper, we presented only the results, giving predictions of what to look for in various ranges of electric and magnetic fields. Here we present the underlying theory. Starting from Kepler action and angle variables, we give a derivation of a classical Hamiltonian to second order in perturbation theory; the derivation is different from, but the final result agrees with, previous work. We focus especially on the topological structure of the reduced phase space and on the resulting topological structure of the trajectories. We show that construction of action variables by the obvious methods leads to variables that have discontinuous derivatives. Smooth continuation of these "primitive" action variables leads to action variables that are multivalued. We show how these multivalued actions lead to lattice defects in the quantum spectrum. Finally we present a few correlation diagrams which show how quantum eigenvalues evolve from one region of near-perpendicular parameter space to another.
\end{abstract}

DOI: 10.1103/PhysRevA.77.043422

PACS number(s): 32.60. $+\mathrm{i}, 32.30 .-\mathrm{r}$

\section{INTRODUCTION}

The hydrogen atom in applied fields has long been a model system for the study of order and chaos in classical and quantum mechanics, because it has just two or three degrees of freedom and a collection of controllable parameters, and because it is accessible to both theory and experiment [1]. The first-order spectrum in combined electric and magnetic fields was examined roughly 80 years ago by Pauli [2] and relatively recently explored in experiments [3]. Recently, much attention has been given to the spectrum at higher order [4], with work closely related to the present work appearing in publications by Uzer, Gourlay, Farrelly, and Milczewski [5-7] and Solov'ev, Herrick, and Braun [8-10]. Publications by Sadovskií and Cushman [11-13] have pointed out that, for exactly perpendicular fields and a certain interval of field strength ratio, the spectrum displays effects of action-angle monodromy.

In a previous paper [14], we showed that, if the fields are tilted slightly away from perpendicular, then the phenomena predicted by Sadovskií and Cushman are modified, and additional phenomena are present at other field ratios. We also indicated how such phenomena are connected with quantized classical actions. Here we present the analysis on which our conclusions were based. Since this analysis is long, and involves concepts that might be unfamiliar, we recommend that the reader first review the results and especially the pictures presented in Ref. [14]. A comparison of the present work with other work appears at the end of Sec. I C. Further perspective on monodromy is given in Refs. [16,17].

\footnotetext{
*crschl@wm.edu; URL: http://www.chrisschleif.com

†jbdelo@wm.edu
}

\section{A. Quantum spectrum}

When the fields are weak and nearly perpendicular, the $n^{2}$ degenerate states at each principal quantum number $n$ are split into $2 n-1$ equally spaced clusters of closely spaced levels [Fig. 1(a)]. The clusters are displaced symmetrically about $E_{o}=-\frac{1}{2 n^{2}}$, and may be labeled by an integer $q$ which ranges from $-(n-1)$ to $n-1$ such that the energy of each state in a cluster is located near

$$
E_{\text {cluster }}=-\frac{1}{2 n^{2}}+\frac{1}{2} \sqrt{B^{2}+(3 n F)^{2}} q
$$

and the cluster contains exactly $n-|q|$ quantum states. The second-order energy $E-E_{\text {cluster }}(n, q)=h_{2}$ is the focus of this paper.

The spectra can be arranged in a lattice if for each state in a given $n$-manifold one plots the second-order energy $h_{2}$ vs $q$. (A more rigorous lattice construction using expectation values of quantum operators is explained in Sec. VII.) For some parameter regimes the resulting lattices may have lattice defects [Fig. 1(b)], double degeneracy, or contain regions which display anticrossings between states of the same $q$ as the fields are varied. Such features can be understood by semiclassical analysis. In this paper the structure of spectral lattices is predicted for the entire near-perpendicular parameter space using Einstein-Brillouin-Keller-Maslov (EBKM) quantization of approximate action variables.

\section{B. Classical trajectories}

Classical trajectories of the electron in a hydrogen atom in sufficiently strong magnetic fields are chaotic. However, if the fields are sufficiently weak, then the trajectories can be described as Kepler ellipses with slowly varying orbital pa- 


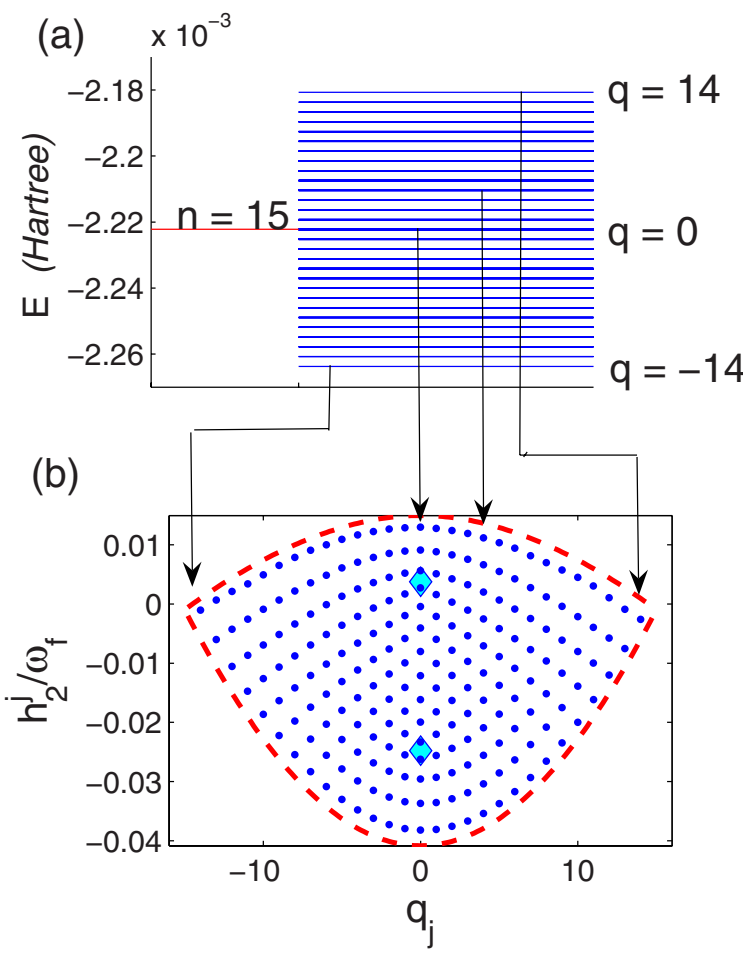

FIG. 1. (Color online) (a) Effects of near-perpendicular fields on the energy spectrum of a spinless hydrogen atom at principal quantum number $n=15$ for electric field $F=11.14 \mathrm{~V} / \mathrm{cm}$ and magnetic field $B=1.393 \mathrm{~T}$ and $\mathbf{F} \cdot \mathbf{B}=0.002 F B$ or $\theta=54^{\circ}, \omega_{f}=2.963 \times 10^{-6}$, and $\chi=0.2 \omega_{f} n^{3}$ ( $\omega_{f}$ has units of a magnetic field and is reported in atomic units in all figures). The quantum basis included $n$-manifolds 12-18. When the fields are turned on, the $n^{2}$-degenerate $n$-manifolds are split into $2 n-1$ equally spaced $q$-manifolds each containing $n-|q|$ levels. The difference between an energy level and the energy of its parent $q$-manifold is denoted $h_{2}$. (b) Structure in the second-order energies in an $n$-manifold is made visible by plotting $h_{2}$ vs $q$ (in all lattice figures we plot $h_{2} / \omega_{f}$ with units of energy/ magnetic field reported in atomic units). At these field parameters (region II) the lattice has two defects associated with the values of $q$ and $h_{2}$ that are marked by (cyan online) diamonds. For various field parameters the structure in the second-order energy spectrum generates various families of spectral lattices. This structure is explained by examining the properties of the classical actions of a Hamiltonian system obtained from Poincaré-Von Zeipel perturbation theory.

rameters. Most of the trajectories are then quasiperiodic, and form three-dimensional tori in the six-dimensional phase space. Classical perturbation theory replaces the exact Hamiltonian of the system with a "nearby" integrable Hamiltonian that has three constants of the motion, and therefore allows only regular trajectories. These three constants of the motion are approximately conserved on the exact trajectories.

When trajectories form continuous families of tori, then local action and angle variables can be constructed, and a semiclassical approximation to the quantum spectrum can be obtained by identifying those tori (sometimes called eigentori) on which the actions are appropriately quantized (usually as integers or half integers). One of the actions is a variable called $Q(\mathbf{r}, \mathbf{p})$, whose numerical value we call $q$,

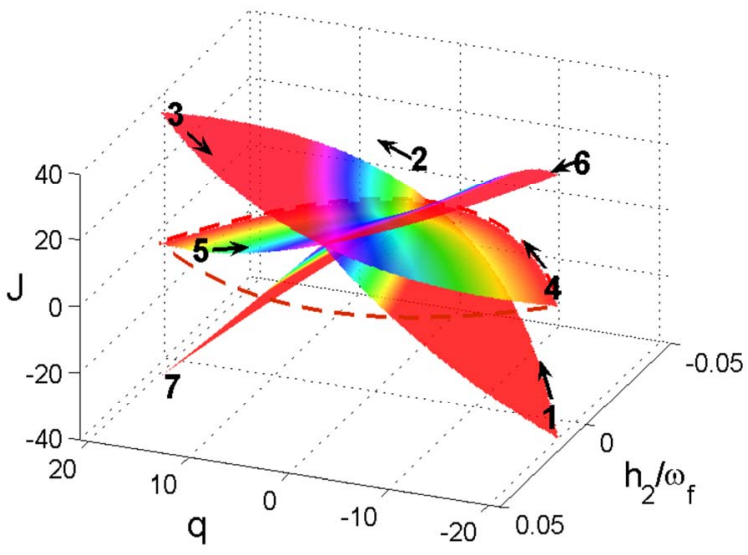

FIG. 2. (Color online) For some field strength ratios, the Hamiltonian system obtained by perturbation theory has an intrinsically multivalued action variable $J\left(q, h_{2}\right)$ [here, $\mathbf{F} \cdot \mathbf{B}=0, n=20$, and $\left.\tan ^{-1}(3 N F / B)=50^{\circ}\right]$. The set of all values of the constants of the motion $q$ and $h_{2}$ that are classically allowed at fixed $n=20$ are contained within the dashed boundary (red online). Almost all points inside this boundary are values of $q$ and $h_{2}$ whose classical level sets are tori, but there is one isolated value of $q$ and $h_{2}$ in the interior whose level set is a pinched torus. Since a full set of actions may be defined only on the tori, $J$ is not defined at this value $(q$ $=0$ and $h_{2} \sim 0.01 \omega_{f}$ ), known as the monodromy center. Away from this point, $J$ is locally smooth everywhere only if it is multivalued, with a branch point at the monodromy center. Three branches of the function are plotted in the figure. To continue the classical action variable smoothly, we may start at (1) and follow the arrows sequentially along the surface all the way to (7); then we pass onto a new branch every time we cross $q=0$ if $h_{2}$ is greater than the energy of the monodromy center.

which is quantized as an integer, and which corresponds to the cluster number in the first-order spectrum.

Two related facts make the second-order spectrum of hydrogen in fields more complex. (1) Not all of the trajectories form tori. As will be explained later, some of them form pinched tori (see figures referenced in Sec. V D), and it is known that pinched tori are associated with Hamiltonian monodromy. (Some more complex structures also occur.) (2) More important from the present perspective, the volume of the reduced phase space is related to $|q|$. Therefore it is not differentiable with respect to $q$ at $q=0$. It follows that one of the action variables (being an integral over a certain area in phase space) is not differentiable at $q=0$. In our formulation, this is the source of monodromy in this system. Action variables by definition are supposed to be smooth functions of phase-space variables and of constants of the motion. If some primitive definition of an action variable gives a discontinuous derivative, then that primitive action should be replaced by a smooth function. In systems having monodromy, smooth actions can be constructed, but they turn out to be multivalued functions of the constants of the motion (Fig. 2). The multivalued gradients of these smooth actions produce lattice defects in the semiclassical spectrum.

\section{Comparison with other work}

The spectrum of hydrogen in fields is an old topic, going back to the earliest developments in quantum theory, and 
studied in many recent papers. It follows that any coherent discussion necessarily contains old results mixed with new ones. Let us briefly survey some recent work to discuss what is new in this paper.

The most important issues that have been treated inadequately (or not at all) in most of the earlier work are the topological aspects of the problem. Some had understood that Kepler averaging reduces the phase space from $\mathbb{R}^{6}$ to $S^{2} \times S^{2}$. However, only recently was it realized that further reduction arising from averaging over the Pauli precession leads to a complex topological structure that contains ordinary tori, but may also contain doubly pinched tori in perpendicular fields [11-13], or singly pinched tori as well as other structures that are connected with monodromy in nearperpendicular fields $[14,15]$ (see figures in Sec. V).

Such phenomena occur at second order in perturbations of combined electric and magnetic fields. To our knowledge, Pauli never went beyond first order. Solov'ev, Uzer, and collaborators and others carried out calculations for crossed fields to second order, but did not make the connection with monodromy, which was not widely understood when they did their work.

There are also several other less important differences between the present work and previous work. (1) Where previous classical perturbation theory made use of the fourdimensional Kustaanheimo-Stiefel regularization [18] and a normal form method [19], we carry out the perturbation in the three-dimensional Delaunay variables. We obtain a second-order Hamiltonian that is different from that obtained by the normal form method until we carry out the Pauli averaging, at which point our result agrees with previous work. To be specific, the resulting intermediate system, describing a small coupling between two independent angular momenta (Pauli's $\mathbf{J}$ momenta), differs from that derived by Gourlay, Uzer, and Farrelly [6] by terms proportional to $F B$ and $F^{2}$ [compare our Eq. (16) with Eq. (38) of Ref. [6]]. However, after averaging over the motion of the Pauli vectors, the resulting system agrees with the previous results of Milczewski and Uzer [7]. We do not know if there is any significance to the discrepancy that exists prior to the Pauli averaging, but it is pleasing to know that the final averaged Hamiltonians obtained by the two different methods agree. Most recently the normal form method was used by Efstathiou, Sadovskií, and Zhilinskií [15] to interpret many of the near-perpendicular spectral structures that we found and displayed in Ref. [14] as well as structures that might be found near resonant angles away from perpendicular fields. We are not able to make an exact comparison between our formulas and theirs, but the structures appearing in Fig. 8 of [15] look like the ones we found in Ref. [14] and here.

(2) We present semiclassical calculations of spectra obtained by EBKM quantization of action variables, and we show that the result agrees with our ab initio quantum calculations, which are based on an expansion of the wave function in a multi- $n$-manifold hydrogenic basis. Milczewski and Uzer [7] did a classical analysis (with less attention to toplogy and no attention to monodromy) but did not quantize to obtain a spectrum. Sadovskií and Cushman [11] obtained a reduced classical Hamiltonian, then converted it to a quantum operator by using a certain quantization postulate, and then constructed an approximate spectrum (such methods are sometimes called "semiquantal"). The connection between the quantum and classical second-order energies presented by Solov'ev [8] is quite different, but may also be called semiquantal and is closely related to works by Herrick [9] and Braun [10]. In these analyses, the quantum Hamiltonian matrix is explicitly obtained and converted to a simple form which may be reexpressed as a recursion relationship. The semiclassical approximations for this recursion relationship yield a classical Hamiltonian. The second-order Hamiltonian obtained by Solov'ev appears in Eqs. (7) and (8) of Ref. [8], and is almost the same as our Hamiltonian [Eq. (12), Sec. II]. The difference is constant at fixed principal quantum number $n$, and can be regarded as a quantum correction to semiclassical theory.

(3) The quantum operators $\omega_{f} \hat{Q}$ and $\omega_{f} \hat{W}$ defined in Sec. VII B are generalizations of previously defined operators to multiple $n$-manifolds (constructed by replacing the quantum number $n^{2}$ with the operator $-\frac{1}{2} \hat{H}_{0}^{-1}$ ). In Sec. IX A we also show that the quantum operator $\hat{W}$ [Eq. (62)] has expectation values that correspond to average values of a corresponding classical variable $W$. Where Solov'ev interpreted some of his results in terms of a "quasibarrier," we have a concrete representation of a boundary between different types of states in the form of a classical separatrix in the reduced phase space.

(4) Finally, there have been a number of papers on the relationship between the quantum spectrum and closed orbits of the electron [20]. The topological aspects (including monodromy) that are discussed here must have an impact on closed-orbit theory, but the implications have not yet been studied.

\section{DEGENERATE PERTURBATION OF THE KEPLER MOTION}

Consider a nonrelativistic, spinless hydrogen atom in static electric and magnetic fields $\mathbf{F}$ and $\mathbf{B}$. Let the $\mathbf{B}$ field vector define the $z$ axis and let the $\mathbf{F}$ and $\mathbf{B}$ field vectors together define the $x-z$ plane. Then for weak, nearly perpendicular fields [21] the Hamiltonian may be written in the following ordering (atomic units):

$$
H=H_{0}+H_{1}+H_{2}=\underbrace{\frac{p^{2}}{2}-\frac{1}{r}}_{H_{0}}+\underbrace{\frac{B}{2} L_{z}+x F_{x}}_{H_{1}}+\underbrace{z F_{z}+\frac{B^{2}}{8}\left(x^{2}+y^{2}\right)}_{H_{2}}
$$

For vanishing field strengths, $H \rightarrow H_{0}$, every bound phasespace orbit with finite energy is periodic. In the Delaunay action-angle coordinates ([22-24] and Table I), the coordinate along this periodic orbit is the principal angle $\phi_{N}$, conjugate to the principal action $N$. We use Poincaré-Von Zeipel degenerate canonical perturbation theory $([22,23])$ to construct an approximate Hamiltonian which is independent of the new principal angle $\bar{\phi}_{N}$ through terms of second order in field strengths. 
TABLE I. Delaunay action and angle variables

\begin{tabular}{lccc}
\hline \hline Canonical angle & Classical name & Conjugate momentum & Classical name \\
\hline$\phi_{N}$ & Mean anomaly & $N$ & Principal action \\
$\phi_{p}$ & Argument of the perihelion & $L$ & Magnitude of the total angular momentum $\mathbf{L}$ \\
$\Omega$ & Longitude of the ascending node & $L_{z}$ & Space-fixed $z$ component of $\mathbf{L}$ \\
\hline \hline
\end{tabular}

\section{A. Canonical perturbation theory}

We first transform the Hamiltonian Eq. (2) from the Cartesian coordinates to the Delaunay action-angle variables. We then enact a time-independent canonical transformation via a generating function $f$ with the following properties: (i) $f$ is expanded in orders of the field strengths about the identity transformation; (ii) $f$ is periodic in both the old and new angles. The canonical transformation relates old and new variables:

$$
\left(N, L, L_{z}, \phi_{N}, \phi_{p}, \Omega\right)_{\text {old }} \leftrightarrow\left(\bar{N}, \bar{L}, \bar{L}_{z}, \bar{\phi}_{N}, \bar{\phi}_{p}, \bar{\Omega}\right)_{\text {new }}
$$

through the generating function

$$
f(q, \bar{P})=q \bar{P}+f_{1}(q, \bar{P})+f_{2}(q, \bar{P})+\cdots .
$$

We refer to the new variables as perturbed Delaunay coordinates. Equating the old and the new Hamiltonians in the space of mixed coordinates $\left(\bar{N}, \bar{L}, \bar{L}_{z}, \phi_{N}, \phi_{p}, \Omega\right)$, we have to second order

$$
\begin{gathered}
\bar{H}_{0}=H_{0} \\
\bar{H}_{1}=H_{1}+\frac{\partial H_{0}}{\partial N} \frac{\partial f_{1}}{\partial \phi_{N}} \\
\bar{H}_{2}=H_{2}+H_{2}^{\prime} \\
H_{2}^{\prime}=\frac{\partial H_{1}}{\partial N} \frac{\partial f_{1}}{\partial \phi_{N}}+\frac{\partial H_{1}}{\partial L} \frac{\partial f_{1}}{\partial \phi_{p}}+\frac{\partial H_{1}}{\partial L_{z}} \frac{\partial f_{1}}{\partial \Omega}+\frac{\partial^{2} H_{0}}{\partial N^{2}}\left(\frac{\partial f_{1}}{\partial \phi_{N}}\right)^{2} \\
+\frac{\partial H_{0}}{\partial N} \frac{\partial f_{2}}{\partial \phi_{N}} .
\end{gathered}
$$

By the imposed $\phi_{N}$ periodicity on the functions $f_{1}$ and $f_{2}$ one obtains

$$
\begin{gathered}
\bar{H}_{0}=H_{0}, \\
\bar{H}_{1}=\left\langle H_{1}\right\rangle_{\phi_{N}}, \\
\bar{H}_{2}=\left\langle H_{2}\right\rangle_{\phi_{N}}+\left\langle H_{2}^{\prime}\right\rangle_{\phi_{N}},
\end{gathered}
$$

where

$$
\langle g\rangle_{\phi_{N}}=\frac{1}{2 \pi} \oint g d \phi_{N} .
$$

The perturbed Delaunay coordinates are related to a new Cartesian space through the same transformation that con- nected the original Delaunay variables and the original Cartesian space. In this new Cartesian space we can consider the Kepler orbits and their associated angular momentum and energy-scaled Laplace-Runge-Lenz eccentricity vectors $\overline{\mathbf{L}}$ and $\overline{\mathbf{M}}$. When a small perturbation is applied to the Kepler system, one may describe the perturbed trajectory as a Kepler ellipse of fixed $\bar{N}$, with slowly varying orbital elements $\overline{\mathbf{L}}$ and $\overline{\mathbf{M}}$. For the remainder of this section we will work exclusively in the new coordinates, and for notational convenience, we now drop the overbars from the new Delaunay variables.

The eccentricity vector $\mathbf{M}$ extends from the nucleus, in the direction of the instantaneous perigee of the ellipse, with magnitude $M=N e$, where $e$ is the eccentricity of the ellipse with $0 \leq e \leq 1$ :

$$
\mathbf{M}=N(\mathbf{p} \times \mathbf{L}-\hat{\mathbf{r}}) .
$$

The angular momentum vector $\mathbf{L}$ extends from the nucleus, normal to the orbital plane,

$$
\mathbf{L}=\mathbf{r} \times \mathbf{p} .
$$

The two vectors are constrained in direction and magnitude by two conditions,

$$
\begin{gathered}
\mathbf{L} \cdot \mathbf{M}=0, \\
\mathbf{L}^{2}+\mathbf{M}^{2}=N^{2},
\end{gathered}
$$

and share a closed Poisson algebra $([25,23])$,

$$
\begin{gathered}
\{\mathbf{L}, \mathbf{L}\}=\epsilon \mathbf{L}, \\
\{\mathbf{M}, \mathbf{M}\}=-\epsilon \mathbf{L}, \\
\{\mathbf{M}, \mathbf{L}\}=\epsilon \mathbf{M}, \\
\left\{\mathbf{L}, H_{0}\right\}=\left\{\mathbf{M}, H_{0}\right\}=0 .
\end{gathered}
$$

Equations (5) and (6) are expressed in Delaunay variables. However, the new Hamiltonian is independent of $\phi_{N}$ by construction, and therefore can depend only on the fixed value of $N$, and on the instantaneous eccentricity and orientation of the ellipse in space. Therefore, it can be expressed as a function of the components of $\mathbf{L}$ and $\mathbf{M}$. We evaluate the averages in Eqs. (6) in an orbital frame defined by the basis vectors

$$
\{\hat{z}, \hat{x}, \hat{y}\}=\left\{\frac{\mathbf{L}}{L}, \frac{\mathbf{M}}{M}, \frac{\mathbf{L} \times \mathbf{M}}{L M}\right\},
$$

and it is shown in Appendix A that the resulting Hamiltonian is 


$$
\begin{aligned}
\bar{H}(\mathbf{L}, \mathbf{M} ; N)= & \bar{H}_{0}+\bar{H}_{1}+\bar{H}_{2}=-\frac{1}{2 N^{2}}+\underbrace{\frac{1}{2} B_{z} L_{z}-\frac{3}{2} N F_{x} M_{x}}_{\left\langle H_{1}\right\rangle_{\phi_{N}}} \\
& +\underbrace{\frac{B^{2} N^{2}}{16}\left(N^{2}+4 M^{2}-5 M_{z}^{2}+L_{z}^{2}\right)-\frac{3}{2} N F_{z} M_{z}}_{\left\langle H_{2}\right\rangle_{\phi_{N}}} \\
& -\underbrace{\frac{F_{x}^{2} N^{4}}{16}\left(29 N^{2}-24 M^{2}-21 L_{x}^{2}+9 M_{t}^{2}\right)}_{\left\langle H_{2}^{\prime} \phi_{\phi_{N}}\right.}
\end{aligned}
$$

This result differs from Eqs. (7) and (8) of Ref. [8] by $19 N^{4} F^{2} / 16$.

The Hamiltonian in Eq. (12) governs the motion of $\mathbf{L}$ and $\mathbf{M}$ at fixed $N$, preserving the constraints in Eqs. (9). That motion is conveniently described using Pauli's $\mathbf{J}$ vectors,

$$
\begin{aligned}
& \mathbf{J}_{1}=\frac{1}{2}(\mathbf{L}+\mathbf{M}), \\
& \mathbf{J}_{2}=\frac{1}{2}(\mathbf{L}-\mathbf{M}) .
\end{aligned}
$$

By the properties [Eqs. (9)] of $\mathbf{L}$ and $\mathbf{M}$ and their Poisson brackets [Eqs. (10)] one may calculate

$$
\begin{array}{r}
\left\{\mathbf{J}_{i}, \mathbf{J}_{i}\right\}=\epsilon \mathbf{J}_{i}, \\
\left\{\mathbf{J}_{i}, \mathbf{J}_{j \neq i}\right\}=0,
\end{array}
$$

and

$$
\left|\mathbf{J}_{1}\right|=\left|\mathbf{J}_{2}\right|=\frac{N}{2} .
$$

The two Pauli vectors have identical fixed magnitudes and may be oriented arbitrarily in space. Every Kepler orbit of a given $N$-manifold is thus identified with a single point on $S^{2} \times S^{2}$; i.e., the reduced phase space is the product of two spheres.

Expressing $\mathbf{L}$ and $\mathbf{M}$ in terms of the $\mathbf{J}$ vectors by inverting Eqs. (13) and substituting in Eq. (12) yields

$$
\begin{aligned}
\bar{H}\left(\mathbf{J}_{1}, \mathbf{J}_{2} ; N\right)= & -\frac{1}{2 N^{2}}+\frac{B}{2}\left(J_{1, z}+J_{2, z}\right)-\frac{3}{2} N F_{x}\left(J_{1, x}-J_{2, x}\right) \\
& -\frac{3}{2} N F_{z}\left(J_{1, z}-J_{2, z}\right)+\frac{B^{2} N^{2}}{16}\left[3 N^{2}+4 J_{1, z} J_{2, z}\right. \\
& \left.-8\left(J_{1, x} J_{2, x}+J_{1, y} J_{2, y}\right)-4\left(J_{1, z}^{2}+J_{2, z}^{2}\right)\right] \\
& -\frac{F_{x}^{2} N^{4}}{16}\left[17 N^{2}+48\left(J_{1, y} J_{2, y}+J_{1, z} J_{2, z}\right)\right. \\
& \left.-12\left(J_{1, x}^{2}+J_{2, x}^{2}+J_{1, x} J_{2, x}\right)\right] .
\end{aligned}
$$

This result differs from Eq. (38) of Ref. [6] by terms proportional to $F_{x} B$ and $F_{x}^{2}$.

\section{FIRST-ORDER DYNAMICS: THE PAULI PRECESSION}

The Hamiltonian Eq. (16) governs a reduced twodimensional system describing the slow evolution of the classical orbital elements. Since $N$ is conserved, $\bar{H}_{0}$ can be regarded as an additive constant. To first order, the fields are perpendicular, and the first-order Hamiltonian is

$$
\bar{H}_{1}=\frac{1}{2} B_{z} L_{z}-\frac{3}{2} N F_{x} M_{x} .
$$

All orbits of $\bar{H}_{1}$ are strictly periodic as will be shown in the following sections.

\section{A. Pauli precession}

For arbitrary orientation of electric and magnetic fields, a calculation of the first-order effects on the hydrogen spectrum is due to Pauli [2]. He defined two "effective field vectors"

$$
\begin{aligned}
& \boldsymbol{\Omega}_{1}=\frac{1}{2} \mathbf{B}-\frac{3}{2} N \mathbf{F}, \\
& \boldsymbol{\Omega}_{2}=\frac{1}{2} \mathbf{B}+\frac{3}{2} N \mathbf{F} .
\end{aligned}
$$

One may define scaled versions of the $\boldsymbol{\Omega}$ vectors such that for exactly perpendicular fields $(\mathbf{F} \cdot \mathbf{B}=0)$ the scaled versions have unit magnitude:

$$
\boldsymbol{\omega}_{j}=\frac{\boldsymbol{\Omega}_{j}}{\omega_{f}}
$$

with

$$
\omega_{f}=\frac{1}{2} \sqrt{B^{2}+(3 N F)^{2}} .
$$

Using Eqs. (13) and (19), Pauli wrote the first-order crossed field Hamiltonian in the form

$$
H_{\text {Pauli }}=\omega_{f}\left(\boldsymbol{\omega}_{1} \cdot \mathbf{J}_{1}+\boldsymbol{\omega}_{2} \cdot \mathbf{J}_{2}\right) .
$$

The form of the Hamiltonian Eq. (20a) and the Poisson algebra of the J's Eqs. (14) imply that the equations of motion describe the precession of $\mathbf{J}_{1}$ about $\omega_{1}$ such that its component $\mu_{1}$ along the $\omega_{1}$ axis is conserved, and an analogous precession of $\mathbf{J}_{2}$ about $\omega_{2}$ conserving $\mu_{2}$. Then the Hamiltonian Eq. (20a) can be rewritten in terms of the components $\mu_{i}$ of the $\mathbf{J}_{i}$ vectors in the $\omega_{i}$ directions:

$$
H_{\text {Pauli }}=\omega_{f}\left(\omega_{1} \mu_{1}+\omega_{2} \mu_{2}\right) .
$$

For exactly perpendicular fields $\omega_{1}$ and $\omega_{2}$ are unity [Eqs. (19)]. This implies that the first-order Hamiltonian Eq. (17) can be expressed as

$$
\bar{H}_{1}=\omega_{f}\left(\mu_{1}+\mu_{2}\right) .
$$

Thus, for perpendicular fields, both vectors precess at the same rate $\omega_{f}$ about their respective axes, and the motion is strictly periodic.

\section{B. Local canonical coordinates on $S^{2} \times S^{2}$}

We now construct a canonical coordinate system to describe the Pauli precession motion. For all $\mu_{1}$ such that $-N / 2<\mu_{1}<N / 2$, there is an angle $\psi_{1}$ with $0<\psi_{1} \leq 2 \pi$ which describes the position of $\mathbf{J}_{1}$ on the cone of precession. This angle may be defined in terms of the vector components 
of $\mathbf{J}_{1}$ in a Cartesian coordinate system having the $z^{\prime}$ axis parallel to $\boldsymbol{\Omega}_{1}$ and the $y^{\prime}$ axis parallel to the $y$ axis in the space-fixed frame such that $J_{1, z^{\prime}}=\mu_{1}$ :

$$
\psi_{1}=\tan ^{-1}\left(\frac{J_{1, y^{\prime}}}{J_{1, x^{\prime}}}\right) .
$$

Similarly, for all $\mu_{2}$ such that $-N / 2<\mu_{2}<N / 2$ there is an angle $\psi_{2}$, describing the precession of $\mathbf{J}_{2}$, which is defined analogously to $\psi_{1}$. Thus, at each $\left(\mu_{1}, \mu_{2}\right)$ with neither $\left|\mu_{1}\right|$ nor $\left|\mu_{2}\right|$ equal to $N / 2$, the available phase space consists of a 2-torus.

It is clear from Eq. (21) that there are some values of the $\mu_{i}=J_{i, z^{\prime}}$ for which this coordinate system breaks down. When $\left|\mu_{i}\right|=N / 2, J_{i, x^{\prime}}=J_{i, y^{\prime}}=0$ and so $\psi_{i}$ is undefined. However, as long as $\left|\mu_{j \neq i}\right| \neq N / 2, \psi_{j}$ is still defined, and the phase space is a circle. When both $\left|\mu_{1}\right|$ and $\left|\mu_{2}\right|$ are equal to $N / 2$, the phase space is a point.

By the properties of the $\mathbf{J}_{1}$ and $\mathbf{J}_{2}$ Poisson algebra Eqs. (14) and the definitions implied by Eq. (21), the two angles $\psi_{i}$ and the corresponding effective field vector components $\mu_{i}$ form a system of local canonical coordinates $\left(\mu_{1}, \mu_{2}, \psi_{1}, \psi_{2}\right)$ on the $S^{2} \times S^{2}$ space of all Kepler orbits at fixed $N$. Their Poisson bracket relations follow from Eqs. (21) and (14):

$$
\begin{gathered}
\left\{\mu_{i}, \psi_{j}\right\}=\delta_{i, j}, \\
\left\{\psi_{i}, \psi_{j}\right\}=0, \\
\left\{\mu_{i}, \mu_{j}\right\}=0 .
\end{gathered}
$$

The local symplectic 2-form associated with Eqs. (22) is [26]

$$
\omega^{2}=d \mu_{1} \wedge d \psi_{1}+d \mu_{2} \wedge d \psi_{2} .
$$

\section{Degenerate coordinates on the Pauli 2-torus}

The first-order canonical equations of motion are obtained from Eq. (20c):

$$
\begin{gathered}
\mu_{j}(t)=\mu_{j}(0), \\
\psi_{j}(t)=\omega_{f} t+\psi_{j}(0),
\end{gathered}
$$

for $j=1,2$. At fixed values of the momenta $\left(\mu_{1}, \mu_{2}\right)$ where both angles are defined, the phase space is a 2-torus covered by the coordinates $\psi_{1}$ and $\psi_{2}$. When the torus is depicted as a square of length $2 \pi$, the motion is along a straight line with a unit slope, reflecting the one-to-one resonance between the angles on the Pauli torus at perpendicular fields.

We make a canonical transformation into coordinates such that one of the new angles $\delta_{Q}$ is aligned along this periodic motion. The transformation $\left(\mu_{1}, \mu_{2}, \psi_{1}, \psi_{2}\right) \rightarrow(Q, W$, $\left.\delta_{Q}, \delta_{W}\right)$ may be enacted with the generating function

$$
G\left(\psi_{1}, \psi_{2}, Q, W\right)=\frac{Q}{2}\left(\psi_{1}+\psi_{2}\right)+\frac{W}{2}\left(\psi_{1}-\psi_{2}\right),
$$

from which one obtains the coordinates

$$
Q=\mu_{1}+\mu_{2},
$$

$$
\begin{gathered}
W=\mu_{1}-\mu_{2}, \\
\delta_{Q}=\frac{1}{2}\left(\psi_{1}+\psi_{2}\right), \\
\delta_{W}=\frac{1}{2}\left(\psi_{1}-\psi_{2}\right),
\end{gathered}
$$

and the Hamiltonian function

$$
\bar{H}_{1}=\omega_{f} Q .
$$

Now a 2-torus formerly labeled by the constant values $\left(\mu_{1}, \mu_{2}\right)$ is labeled by the constant values $(Q, W)$. From Eqs. (23) and (25) it follows that in the new coordinates the local canonical 2 -form is

$$
\omega^{2}=d Q \wedge d \delta_{Q}+d W \wedge d \delta_{W} .
$$

Since $\left|\mathbf{J}_{i}\right|=N / 2$, the allowed values of the components $\mu_{1}$ and $\mu_{2}$ form a closed square with length $[-N / 2, N / 2]$. It follows that the allowed ranges of $Q$ and $W$ become $-N$ $\leq Q \leq N$ and $-(N-|Q|) \leq W \leq(N-|Q|)$ as is illustrated in Fig. 3(a). The allowed ranges of $\delta_{Q}$ and $\delta_{W}$ can be chosen in a number of ways. For values of $Q\left(\mu_{1}, \mu_{2}\right)$ and $W\left(\mu_{1}, \mu_{2}\right)$ such that both $\psi_{1}$ and $\psi_{2}$ are defined, they are coordinates on a 2 -torus $\bmod (2 \pi)$, and it is convenient initially to say that each ranges between $-\pi$ and $\pi$. Then, $\delta_{Q}$ would also range from $-\pi$ to $\pi$, while $\delta_{W}$ would range from $-\left(\pi-\left|\delta_{Q}\right|\right)$ to $\left(\pi-\left|\delta_{Q}\right|\right)$. This, however, is inconvenient. It is better to tile the $\left(\psi_{1}, \psi_{2}\right)$ plane as indicated in Fig. 3(b) such that $\delta_{Q}$ and $\delta_{W}$ have the independent ranges $-\pi \leq \delta_{Q} \leq \pi$ and $-\pi / 2$ $\leq \delta_{W} \leq \pi / 2$.

It follows from our discussion of the breakdown of the $(\mu, \psi)$ coordinates that not all values of $(Q, W)$ label a 2-torus. If $\mu_{1}$ and $\mu_{2}$ are such that one or both $\psi$ 's is undefined, the functions $\delta_{Q}$ and $\delta_{W}$ become meaningless. Thus we cannot use these coordinate functions at any point such that $|W|=N-|Q|$.

\section{DEGENERATE PERTURBATION OF THE PAULI MOTION}

In the previous section it was shown that, to first order in field strengths and angle, the principal effect of the perturbation is a periodic evolution $\delta_{Q}$ at fixed $N, Q, W$, and $\delta_{W}$. To describe the effects of second-order terms in the Hamiltonian, we use classical canonical degenerate perturbation theory a second time, effectively reducing the system to a single degree of freedom.

The Hamiltonian $\bar{H}$ in Eq. (16) is a function of the spacefixed components of the Pauli vectors $J_{i, x_{j}}$ [Eqs. (13)] in the space-fixed basis $(\hat{x}, \hat{y}, \hat{z})$. In a reference frame specified by the basis vectors $\left(\hat{x}_{i}^{\prime} \equiv \hat{y} \times \hat{\omega}_{i}, \hat{y}_{i}^{\prime} \equiv \hat{y}, \hat{z}_{i}^{\prime} \equiv \hat{\omega}_{i}\right)$ the components of the Pauli vectors $J_{i, x_{j}^{\prime}}$ are expressed as functions of $\mu_{i}$ and $\psi_{i}:$

$$
\left(J_{i, x^{\prime}}, J_{i, y^{\prime}}, J_{i, z^{\prime}}\right)=\left(\sqrt{\frac{N^{2}}{4}-\mu_{i}^{2}} \cos \psi_{i}, \sqrt{\frac{N^{2}}{4}-\mu_{i}^{2}} \sin \psi_{i}, \mu_{i}\right) .
$$

These vector components are then written as functions of the coordinates $\left\{Q, W, \delta_{Q}, \delta_{W}\right\}$ using the transformations in Eqs. 
(a)

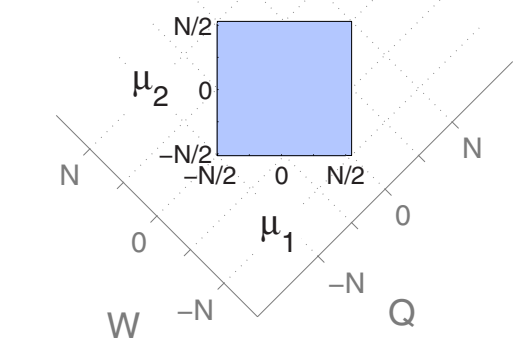

(b)

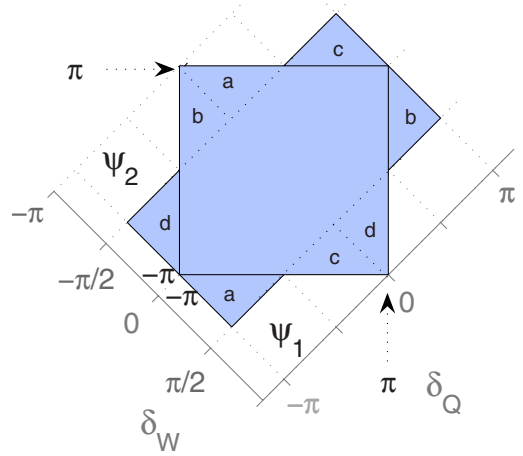

FIG. 3. (Color online) Transformation of the coordinate ranges. (a) The set of all possible components $\mu_{i}$ of $\mathbf{J}_{i}$ along $\omega_{i}$, for $\left|J_{i}\right|$ $=N / 2$ and $i=1,2$, define a closed square. The introduction of the coordinates $Q=\mu_{1}+\mu_{2}, W=\mu_{1}-\mu_{2}$ implies the ranges $-N \leq Q \leq N$ and $-(N-|Q|) \leq W \leq N-|Q|$. (b) $\psi_{1}$ and $\psi_{2}$ are angular coordinates on a 2-torus, defined $\bmod (2 \pi)$. The ranges of $\delta_{Q}=\left(\psi_{1}+\psi_{2}\right) / 2$ and $\delta_{W}=\left(\psi_{1}-\psi_{2}\right) / 2$ are inconvenient when restricted to the square indicated in the figure. Since the square is simply a particular choice of tiling the 2-torus, we are free to retile the plane such that the angles have rectangular restrictions. The pairs of triangular regions marked with identical letters contain points in the plane which represent identical points on the torus. We see that the torus can be described by the angles $-\pi<\delta_{Q} \leq \pi$ and $-\pi / 2<\delta_{W} \leq \pi / 2$.

(26). The space-fixed components of $\mathbf{J}_{i}$ are related to its $\omega_{i}$ frame components by the orthonormal transformation matrix $M_{i}$ :

$$
J_{i, \alpha}=M_{i, \alpha \beta^{\prime}} J_{i, \beta^{\prime}},
$$

where $\alpha=x, y, z, \beta^{\prime}=x^{\prime}, y^{\prime}, z^{\prime}, i=1,2$, and

$$
M_{i, j k}=\hat{x}_{j} \cdot \hat{x}_{k}^{\prime} \text {. }
$$

We substitute the resulting expressions $J_{i, x_{j}}\left(Q, W, \delta_{Q}, \delta_{W}\right)$ for the space-fixed components into Eq. (16) to obtain

$$
\bar{H}=-\frac{1}{2 N^{2}}+\omega_{f} Q+\bar{H}_{2}\left(Q, W, \delta_{Q}, \delta_{W}\right) .
$$

We then enact the canonical transformation

$$
\left(Q, W, \delta_{Q}, \delta_{W}\right) \rightarrow\left(\tilde{Q}, \widetilde{W}, \widetilde{\delta}_{Q}, \widetilde{\delta}_{W}\right),
$$

using a near-identity generating function

$$
g(q, \widetilde{P})=q \widetilde{P}+g_{1}(q, \widetilde{P}) .
$$

Since $g_{1}$ must be periodic in $\delta_{Q}$, one obtains expressions for the new Hamiltonian $h$,

$$
\begin{gathered}
h_{0}+h_{1}=\bar{H}_{0}+\bar{H}_{1}, \\
h_{2}=\left\langle\bar{H}_{2}\right\rangle_{\delta_{Q}},
\end{gathered}
$$

where

$$
\langle f\rangle_{\delta_{Q}}=\frac{1}{2 \pi} \oint f d \delta_{Q} .
$$

The result is a Hamiltonian $h=h_{0}+h_{1}+h_{2}$ which is independent of $\widetilde{\delta}_{Q}$.

\section{A. The fully reduced Hamiltonian}

It is straightforward to compute $\left\langle\bar{H}_{2}\right\rangle_{\delta_{O}}$, and one obtains (dropping all decorations in the final coordinate system)

$$
\begin{aligned}
h_{2}= & h_{c}+\beta W^{2}+\zeta W \\
& +\alpha \sqrt{\left[N^{2}-(Q-W)^{2}\right]\left[N^{2}-(Q+W)^{2}\right]} \cos \left(2 \delta_{W}\right),
\end{aligned}
$$

where each of the coefficients $\alpha, \beta$, and $\zeta$ is second order in the field strengths,

$$
\begin{gathered}
\alpha \equiv-\frac{N^{2}}{16} 2 B^{2}, \\
\beta \equiv-\frac{N^{2}}{16}\left(B^{2}-\left(3 N F_{x}\right)^{2}+\frac{2 B^{4}}{B^{2}+\left(3 N F_{x}\right)^{2}}\right), \\
\zeta \equiv-\frac{3 N F_{z} B}{2 \sqrt{B^{2}+\left(3 N F_{x}\right)^{2}}} .
\end{gathered}
$$

$h_{c}$ is independent of the coordinates $\left(W, \delta_{W}\right)$, and may be regarded as another additive constant,

$$
\begin{aligned}
h_{c}= & \frac{N^{2}}{16}\left[\frac{B^{2}}{3}\left(7+\frac{2 B^{2}}{B^{2}+\left(3 N F_{x}\right)^{2}}\right)-17 N^{2} F_{x}^{2}\right] N^{2} \\
& +\frac{N^{2}}{16}\left[\frac{\left(3 N F_{x}\right)^{2}}{3}+B^{2}\left(1-\frac{2 B^{2}}{B^{2}+\left(3 N F_{x}\right)^{2}}\right)\right] Q^{2} .
\end{aligned}
$$

$h_{2}$ is independent of $\delta_{Q}$, rendering $Q$ a constant of the motion, and we have obtained a system with one degree of freedom governed by the effective Hamiltonian $h_{2}\left(W, \delta_{W}\right)$.

\section{B. Expression in scaled parameters}

The Hamiltonian in Eqs. (35) can be expressed in terms of $\omega_{f}$ defined in Eq. (19b) such that $\left|h_{2}\right| \approx \omega_{f}^{2}$. The following definition of $\theta$ allows a convenient parametrization of all possible field magnitude ratios at a given perturbation strength $\omega_{f}$ : 


$$
\theta=\tan ^{-1}\left(\frac{3 N|F|}{|B|}\right), \quad 0 \leq \theta \leq \frac{\pi}{2} .
$$

This implies the following two field definitions:

$$
\begin{gathered}
3 N F=2 \omega_{f} \sin \theta, \\
B=2 \omega_{f} \cos \theta
\end{gathered}
$$

with the Zeeman and Stark limits at $\theta=0$ and $\theta=\pi / 2$, respectively. The angle between the electric field and the $x$ axis is $\chi$, and implies that $F \sin \chi=F_{z}$. Then Eq. (35b) is rewritten using Eq. (37b):

$$
\alpha=-\frac{N^{2} \omega_{f}^{2}}{4} 2 \cos ^{2} \theta
$$

$\beta, \zeta$, and $h_{c}$ in Eqs. (35c)-(35e) depend on the quantity $\left(3 N F_{x}\right)^{2}$. For near-perpendicular fields such that $|\chi| \lesssim \omega_{f} n^{3}$ $\ll 1$, we may replace $F_{z}$ with $\chi F$ and $\left(3 N F_{x}\right)^{2}$ with $(3 N F)^{2}$ with negligible error at second order. Using these replacements, along with Eqs. (37), we rewrite Eqs. (35c)-(35e) as

$$
\begin{gathered}
\beta=\frac{N^{2} \omega_{f}^{2}}{4}\left[1-2\left(\cos ^{2} \theta+\cos ^{4} \theta\right)\right], \\
\zeta=-\frac{N^{2} \omega_{f}^{2}}{4}\left(4 \cos \theta \sin \theta \frac{\chi}{\omega_{f} N^{2}}\right), \\
h_{c}=\frac{N^{2} \omega_{f}^{2}}{4}\left(-\frac{17}{9}+\frac{38}{9} \cos ^{2} \theta+\frac{2}{3} \cos ^{4} \theta\right) N^{2} \\
+\frac{N^{2} \omega_{f}^{2}}{4}\left(\frac{1}{3}+\frac{2}{3} \cos ^{2} \theta-2 \cos ^{4} \theta\right) Q^{2} .
\end{gathered}
$$

\section{REDUCED DESCRIPTIONS}

In this section we display the topological structure of the reduced phase spaces.

\section{A. Structure of the four-dimensional reduced phase space $\boldsymbol{\Gamma}_{N}$}

The space of all Kepler orbits at a fixed $N$ is denoted $\Gamma_{N} \sim S^{2} \times S^{2}$, and each of the points $\left(\mathbf{J}_{1}, \mathbf{J}_{2}\right)$ in this fourdimensional reduced phase space represents a Kepler orbit. The local canonical coordinates in $\Gamma_{N}$ are $\left(Q, W, \delta_{Q}, \delta_{W}\right)$. We organize the structure of $\Gamma_{N}$ by considering the subset of phase space that is located at each value of $Q$ and $W$ (Fig. 4). At each $(Q, W)$ with $|Q|<N$ and $|W|<(N-|Q|)$, there is a 2-torus with coordinates $\delta_{Q}$ and $\delta_{W}$. At points $0<|Q|<N$, $W= \pm(N-|Q|)$ there is a circle [27]. At each of the four corners $(Q= \pm N, W=0)$ and $(Q=0, W= \pm N)$, there is only a point. At every value of $Q$ and $W$, we call the phasespace orbit generated by the Hamiltonian Eq. (20c) the Pauli orbit at fixed $Q, W$ and also fixed $\delta_{W}$ if $|W|<N-|Q|$. A Pauli orbit is a one-dimensional closed curve everywhere except at the four corners, where it is a point.

The second-order field terms act as a small perturbation to the Pauli system described in Sec. III. One may describe the

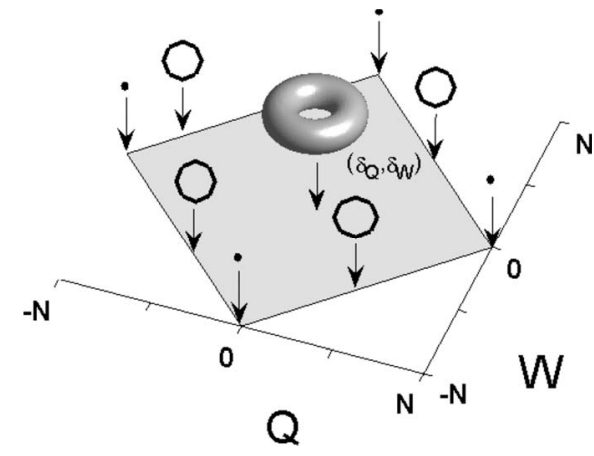

FIG. 4. The structure of $\Gamma_{N}$, the four-dimensional reduced phase space at fixed $N$. Each base point $(Q, W)$ with $|Q|<N$ and $|W|$ $<(N-|Q|)$ labels a 2-torus with coordinates $\delta_{Q}$ and $\delta_{W}$. The points $0<|Q|<N, W= \pm(N-|Q|)$ correspond to a circle. At each of the four corners $(Q= \pm N, W=0)$ and $(Q=0, W= \pm N)$, the phase space consists of a single point. At each fixed $Q, W$, and fixed $\delta_{W}$ if $|W|<N-|Q|$, there is a periodic orbit, which we call the Pauli orbit, generated by the Hamiltonian $H=\omega_{f} Q=\omega_{f}\left(\mu_{1}+\mu_{2}\right)$. Each Pauli orbit is a circle except at the four corners, where it is a point.

resulting motion as a Pauli orbit of fixed $Q$, with slowly varying orbital elements $W$ and $\delta_{W}$.

Three distinct structures of phase space result from fixing particular values of $Q$ on the closed interval $[-N, N]$ (see Fig. 4). (i) The trivial case is obtained by fixing $Q= \pm N$, where phase space is a point. (ii) Fixing any $Q$ such that $0<|Q|$ $<N$ results in a structure which is depicted in Fig. 5(a). $W$ ranges between $\pm(N-|Q|)$, and for all $|W|<N-|Q|$ there is a 2-torus with coordinates $\delta_{Q}$ and $\delta_{W}$, which collapses to a circle at either $W= \pm(N-|Q|)$. A circle at every $W$ is identified with a Pauli orbit. For $|W|<N-|Q|$, the Pauli Hamiltonian $H=\omega_{f} Q=\omega_{f}\left(\mu_{1}+\mu_{2}\right)$ generates the circle with coordinate $\delta_{Q}$, and at $W= \pm(N-|Q|)$, the Pauli orbit is along the appropriate $\psi_{i}$. For convenience we define $\sigma_{Q}$ to be equal to $\delta_{Q}$ for $|W|<N-|Q|$, and to be equal to the appropriate $\psi_{i}$ for $W= \pm(N-|Q|)$. (iii) The case of $Q=0$ is illustrated in Fig. 5(b). $W$ ranges between $\pm N$, and for all $|W|<N$ there is a 2-torus with coordinates $\delta_{Q}$ and $\delta_{W}$, which collapses to a point at either $W= \pm N$. For $|W|<N$, the Pauli orbit is the circle with coordinate $\delta_{Q}$, and at $W= \pm N$, the Pauli orbit consists of a single point in $\Gamma_{N}$.

For the nontrivial cases (ii) and (iii), when we consider each Pauli orbit at fixed $N$ and fixed $Q$ to be a point in a fully reduced phase space, then that space is a two-dimensional surface denoted $\Gamma_{N, Q}$, which is connected like a sphere (Appendix B) and is equipped with the local canonical coordinates $\left(W, \delta_{W}\right)$.

Here we must caution the reader about a subtle point. In the four-dimensional phase space $S^{2} \times S^{2}$, the twodimensional surface defined by constant $Q$ and constant $\sigma_{Q}$ and spanned by $\left(W, \delta_{W}\right)$ is not homeomorphic to a sphere. It is only after we regard all points $\sigma_{Q}\left(0 \leq \sigma_{Q}<2 \pi\right)$ as being equivalent that the $\left(W, \delta_{W}\right)$ surface called $\Gamma_{N, Q}$ is homeomorphic to a sphere.

\section{B. Two-dimensional fully reduced phase space $\boldsymbol{\Gamma}_{N, Q}$}

The total energy of the system is the value of the Hamiltonian $h$, 


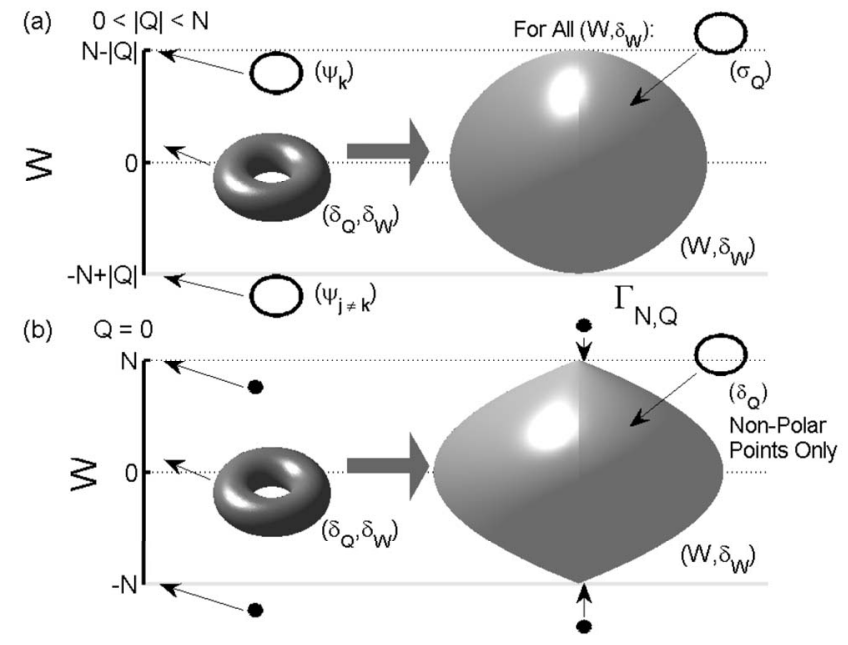

FIG. 5. The structure of the reduced phase space at fixed $N$ and fixed $Q$, and the space of all Pauli orbits $\Gamma_{N, Q}$. The values of $W$ range between $\pm(N-|Q|)$. For all $W$ such that $|W|<N-|Q|$, there exists a 2-torus with coordinates $\delta_{Q}$ and $\delta_{W}$. On each torus, the $\delta_{Q}$ circle is identified with the Pauli orbit, generated by the Hamiltonian $H=\omega_{f}\left(\mu_{1}+\mu_{2}\right)$. (a) For a fixed $Q$ with $0<|Q|<N$, there exists a circle at the end points $W= \pm(N-|Q|)$, identified with the Pauli orbit, and having the appropriate $\psi_{i}$ coordinate. (b) For $Q$ $=0$ there is only a single point at $W= \pm N$, each identified with a stationary Pauli orbit. (a),(b) (right) For all fixed $N$ and $Q \neq \pm N$, the space of all Pauli orbits $\Gamma_{N, Q}$ is a two-dimensional surface that is connected like a sphere and has the coordinates $W$ and $\delta_{W}$. At every point on this surface there exists a Pauli orbit which is (a) everywhere a circle for $0<|Q|<N$, but (b) for $Q=0$ a circle at all nonpolar points, but a point at the poles.

$$
h=-\frac{1}{2 N^{2}}+\omega_{f} Q+h_{2}\left(W, \delta_{W} ; N, Q\right),
$$

where $h_{2}$ is defined in either Eqs. (35) or Eqs. (38). Since $h_{2}$ conserves $N$ and $Q$ and is independent of $\delta_{Q}$, the dynamics are described by the motion of the system point on the $\left(\mathrm{W}, \delta_{W}\right)$ surface $\Gamma_{N, Q}$. The system point will evolve on this surface according to Hamilton's equations of motion,

$$
\begin{gathered}
\frac{d W}{d t}=-\frac{\partial h_{2}}{\partial \delta_{W}}, \\
\frac{d \delta_{W}}{d t}=\frac{\partial h_{2}}{\partial W} .
\end{gathered}
$$

Therefore, all information about the total energy [Eq. (39)], and the dynamics of the second-order system [Eqs. (40)], are ascertained by inspection of the contours of $h_{2}$ on the $\left(W, \delta_{W}\right)$ surface at fixed $N$ and $Q$.

On $\Gamma_{N, Q}$ the canonical 2-form in Eq. (28) is

$$
\omega^{2}=d W \wedge d \delta_{W} .
$$

Equation (41) is not defined at the poles $[W= \pm(N-|Q|)]$, but we will be interested only in surface integrals of Eq. (41), and not the 2-form itself.

To describe the dynamics of the reduced system, it is useful to depict the $\left(W, \delta_{W}\right)$ surface as a two-dimensional sur- face in a three-dimensional space in several different ways (Fig. 6).

(1) The $\left(W, \delta_{W}\right)$ surface may be depicted as a cylinder of radius $\rho=\frac{1}{2}$ and height $L=2(N-|Q|)$. Then the canonical 2 -form [Eq. (41)] is equal to the differential of the Euclidean area on the cylinder's surface, $\omega^{2}=d A_{\text {cyl }}$. However, the boundaries of this cylinder must be understood to each represent a single point on the $\left(W, \delta_{W}\right)$ surface (the north and south poles). Cutting this cylinder along $\delta_{W}= \pm \pi / 2$ and unrolling the surface yields flat contour maps of $h_{2}$, which appear in this paper and in Ref. [14]. In these flat representations, (i) the area is the canonical area, (ii) left and right edges where $\delta_{W}= \pm \pi / 2$ are identified, and (iii) the upper and lower boundaries where $W= \pm(N-|Q|)$ are understood to each consist of a single point. The cylinder representation is useful for calculating actions, and for depicting the phase space on a page, but it can obscure the behavior near the poles $W= \pm(N-|Q|)$.

(2) As an alternative, the $\left(W, \delta_{W}\right)$ surface may be depicted as a sphere [28] of radius $r=N-|Q|$ in a fictitious Cartesian space $\left(\xi_{1}, \xi_{2}, \xi_{3}\right)$, with $W=\xi_{3}$ and $\delta_{W}=\frac{1}{2} \tan ^{-1}\left(\xi_{2} / \xi_{1}\right)$. The space of Pauli orbits at any fixed $Q$ with $|Q|<N$ is homeomorphic to this sphere, and the canonical 2-form is related to the differential of Euclidean surface area by $\omega^{2}=\frac{1}{2} d A_{\mathrm{sph}} /(N$ $-|Q|)$.

(3) Finally, Sadovskií and Cushman [11-13] have proposed that the differential structure (smoothness) of the reduced phase space is represented by embedding that space in the coordinates $\left(\pi_{1}, \pi_{2}, \pi_{3}\right)$, where

$$
\begin{gathered}
\pi_{1}=W, \\
\pi_{2}=4\left(J_{1, x_{1}^{\prime}}, J_{2, x_{2}^{\prime}}+J_{1, y_{1}^{\prime}}, J_{2, y_{2}^{\prime}}\right), \\
\pi_{3}=4\left(J_{1, y_{1}^{\prime}}, J_{2, x_{2}^{\prime}}-J_{1, x_{1}^{\prime}}, J_{2, y_{2}^{\prime}}\right) .
\end{gathered}
$$

In this space, the $\left(W, \delta_{W}\right)$ surface takes the form of a surface of revolution defined by $\left(\pi_{2}, \pi_{3}\right)=\left(\rho \cos \left(2 \delta_{W}\right), \rho \sin \left(2 \delta_{W}\right)\right)$ with radius function

$$
\rho=\sqrt{\left[N^{2}-(Q+W)^{2}\right]\left[N^{2}-(Q-W)^{2}\right]} .
$$

For $0<|Q|<N$ this surface is smoothly embedded. However, if $Q=0$ this surface is not smoothly embedded, but is pointed at the poles such that in a small neighborhood of $(W= \pm N)$ the embedded $\left(W, \delta_{W}\right)$ surface is a cone [Fig. 5(b), right].

Both the cylinder with ends identified and the $Q=0$ surface in Eq. (3) are homeomorphic to the sphere.

\section{Definition of the classical spectrum}

The classical (second-order) spectrum [29] at a given $N$ is defined by the set of all admissible values of $Q$ and $h_{2}$. Since $Q$ is restricted such that $-N \leq Q \leq N$, the spectrum is confined to lie within this interval. At fixed $N$ and $Q$, the upper and lower boundaries of the spectrum are given by the maximum and minimum values of $h_{2}$, which is a continuous and bounded function on the $\left(W, \delta_{W}\right)$ surface. It follows that the classical spectrum is a closed and bounded subsection of the 
(a)

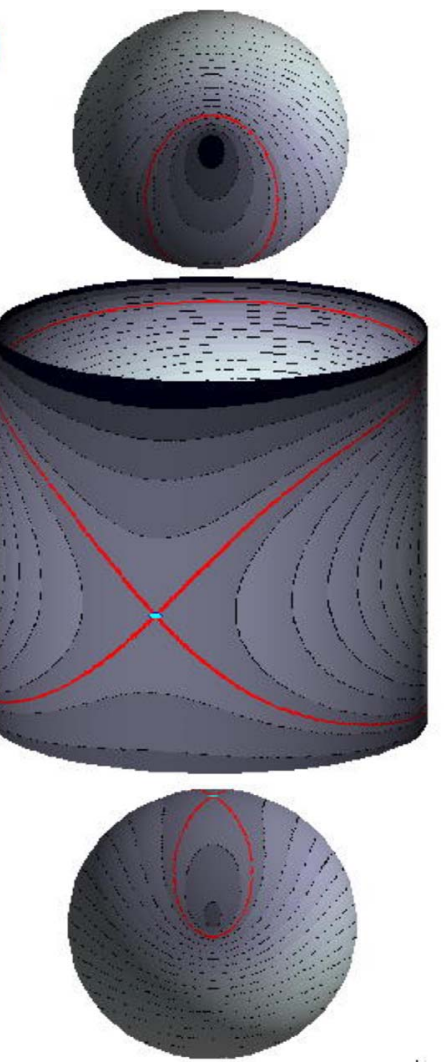

(B)

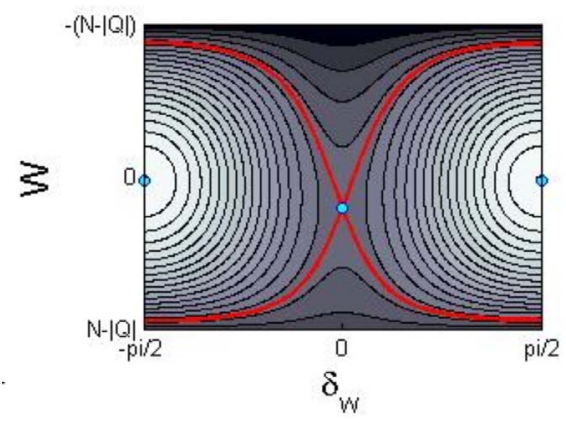

(C)

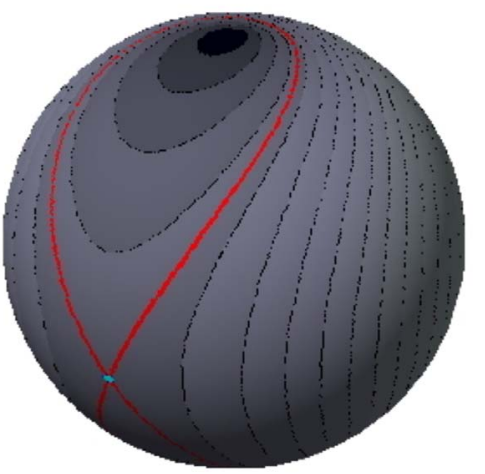

FIG. 6. (Color online) The $\left(W, \delta_{W}\right)$ surface $\Gamma_{N, Q}$ is depicted as a two-dimensional surface in a fictitious three-dimensional space in two ways. The canonical coordinates on $\Gamma_{N, Q}$ are $W$ with $-(N-|Q|) \leq W \leq(N-|Q|)$, and a $\pi$-periodic coordinate $\delta_{W}$ with $-\pi / 2 \leq \delta_{W}<\pi / 2$ [undefined at $W= \pm(N-|Q|)$ ]. (a) The canonical 2-form appropriate for the primitive action integrals is $d W \wedge d \delta_{W}$ and is equal to the differential of the surface area of the cylinder of radius $\rho=1 / 2$ and height $L=2(N-|Q|)$. Cutting this cylinder along $\delta_{W}= \pm \pi / 2$ and unrolling the surface yields the flat contour map in (b). The upper and lower boundaries of the cylinder each represent a single point. Such is the topology of a surface of a sphere. (c) $\Gamma_{N, Q}$ is depicted by a sphere of radius $N-|Q|$ with $W=\xi_{3}$ and $\delta_{W}=\tan ^{-1}\left(\xi_{2} / \xi_{1}\right) / 2$. However, the differential of the spherical surface area is $2(N-|Q|) d W \wedge d \delta_{W}$. On the sphere, all contours that do not contain a fixed point may be regarded as librators, but in the flat representation, the contours can form either apparent librators or apparent rotators.

$\left(Q, h_{2}\right)$ plane. To every point $\left(Q, h_{2}\right)$ in the classical spectrum there corresponds a joint level set in the four-dimensional phase space $\Gamma_{N}$, and $\Gamma_{N}$ is the union of those level sets. Each level set may be connected or disconnected, and we define the classical degeneracy of the joint level set of $\left(Q, h_{2}\right)$ as the number of its disjoint components. In Fig. 6 each joint level set having $h_{2}$ less than the energy of the $\mathrm{x}$ point corresponds to two contours (a "northern" and a "southern"), and is classically doubly degenerate.

\section{Connected components of the $\left(Q, h_{2}\right)$ level sets}

For many points of the spectrum, each connected component of the corresponding $\left(Q, h_{2}\right)$ joint level set in $\Gamma_{N}$ is a 2-torus. Each individual 2-torus has the coordinates $\sigma_{Q}$ (see Sec. V A), and a second periodic coordinate $\sigma_{W}$ which increases uniformly with time around the $h_{2}$ contour on the
$\left(W, \delta_{W}\right)$ surface. However, for some level sets, one or more of their connected components may not be a 2-torus. A component of the $\left(Q, h_{2}\right)$ joint level set is not a 2-torus if its intersection with the $\left(W, \delta_{W}\right)$ surface (i) contains a relative fixed point where Eqs. (40) vanish, or (ii) passes through one of the four points $(Q, W)$ where the Pauli orbit degenerates to a point (at $Q= \pm N$, or $Q=0$ and $W= \pm N$; see Fig. 4). All spectrum points $\left(Q, h_{2}\right)$ for which the joint level set is not composed entirely of 2-tori are marked on the plots of classical spectra with either dashed (red or magenta online) curves or (cyan online) diamonds [30]. (i) and (ii) imply that when a component of a joint level set is not a 2-torus, its structure may be discerned from its intersection with $\Gamma_{N, Q}$.

For components that contain a nonpolar relative fixed point on $\Gamma_{N, Q}$, its structure is determined by analysis of the linear stability matrix. Its eigenvalues are given by

$$
\lambda_{ \pm}= \pm 2|\alpha| \sqrt{2 \cos \left(2 \delta_{W}\right)} \sqrt{\frac{\beta}{\alpha} \Lambda+\cos \left(2 \delta_{W}\right)\left(3 W^{2}-N^{2}-Q^{2}-\frac{2 W^{2}\left(W^{2}-N^{2}-Q^{2}\right)^{2}}{\Lambda^{2}}\right)},
$$




$$
\Lambda=\sqrt{\left[N^{2}-(Q+W)^{2}\right]\left[N^{2}-(Q-W)^{2}\right]} .
$$

When Eq. (42) is imaginary (real) the relative fixed point is an o point (x point).

Components of $\left(Q, h_{2}\right)$ level sets with $0<|Q|<N$ that appear as an o point on the $\left(W, \delta_{W}\right)$ surface have the simple structure of a smooth 1-torus (a Pauli orbit). However, components whose intersection contains an x point have a complex structure resembling a figure eight crossed with a circle.

Additional types of complex components are found at $Q$ $=0$. In Sec. V A we determined that, for $Q=0$, the Pauli orbit at either pole $(W= \pm N)$ is a point. Therefore, any component of a $\left(Q=0, h_{2}\right)$ level set that contains a pole cannot be a 2-torus. For some $\mathbf{F}$ and $\mathbf{B}$, the value of $h_{2}(Q=0, W$ $= \pm N)$ is a local extremum, and these points are isolated, effectively forming o points [31] at a pole of $\Gamma_{N, Q}$. However, for some field parameters, there are components of level sets at $Q=0$ which form an extended contour passing through one or both of the poles of the $\left(W, \delta_{W}\right)$ surface. Since there are $\delta_{Q}$ rings present at all points of the contour except the poles, any such component of a level set must contract to a point as it passes through either pole. A component of a level set of $\left(Q, h_{2}\right)$ which passes through either $W=N$ or $W=-N$ or both, is a pinched 2-torus, with a pinch point at each pole it passes through (Fig. 7).

\section{MONODROMY OF ACTIONS}

In this section we examine the properties of the classical actions as functions of $Q$ and $h_{2}$. Those $\left(Q, h_{2}\right)$ level sets in $\Gamma_{N}$ with components that are 2-tori may form (a) a single simply connected family; (b) a single multiply connected family; (c) two (or more) disjoint families, each of which may be either simply or multiply connected. In a sufficiently small neighborhood of $\Gamma_{N}$ about any one 2-torus, one can always construct local smooth action and angle variables [32]. However, we must carefully distinguish between locally smooth action variables and what we refer to as primitive action integrals. The primitive action integrals are simply areas on Poincaré surfaces divided by $2 \pi$. However, for some $\mathbf{F}$ and $\mathbf{B}$, these are not smooth functions of $Q$ and $h_{2}$.

In this section we will first compute the two primitive action integrals associated with each 2-torus, and find that they have discontinuous derivatives as functions of the constants of the motion. We then show how to obtain smooth action variables from the primitive actions by continuation. The resulting functions are locally smooth functions of the constants of the motion, but if a family of tori is multiply connected, it may be impossible to construct a single-valued and smooth action variable on the entire family. When this is the case, smooth continuation of a local action variable leads to a globally multivalued function on the classical spectrum. Whenever smooth continuation of an action variable around some closed loop in the classical spectrum brings that action variable back to a new value, the system is said to have "nontrivial monodromy of action and angle variables," or simply "monodromy."

\section{A. Primitive action integrals}

To construct primitive actions, we consider the intersection of each $\left(Q, h_{2}\right)$ 2-torus with two fixed Poincaré surfaces, and integrate the canonical 2-form Eq. (41) over the area in the Poincaré surface that is bounded by the intersection contour. The two Poincaré surfaces are chosen to be (i) the $\left(Q, \sigma_{Q}\right)$ surface, and (ii) the $\left(W, \delta_{W}\right)$ surface. By inspecting
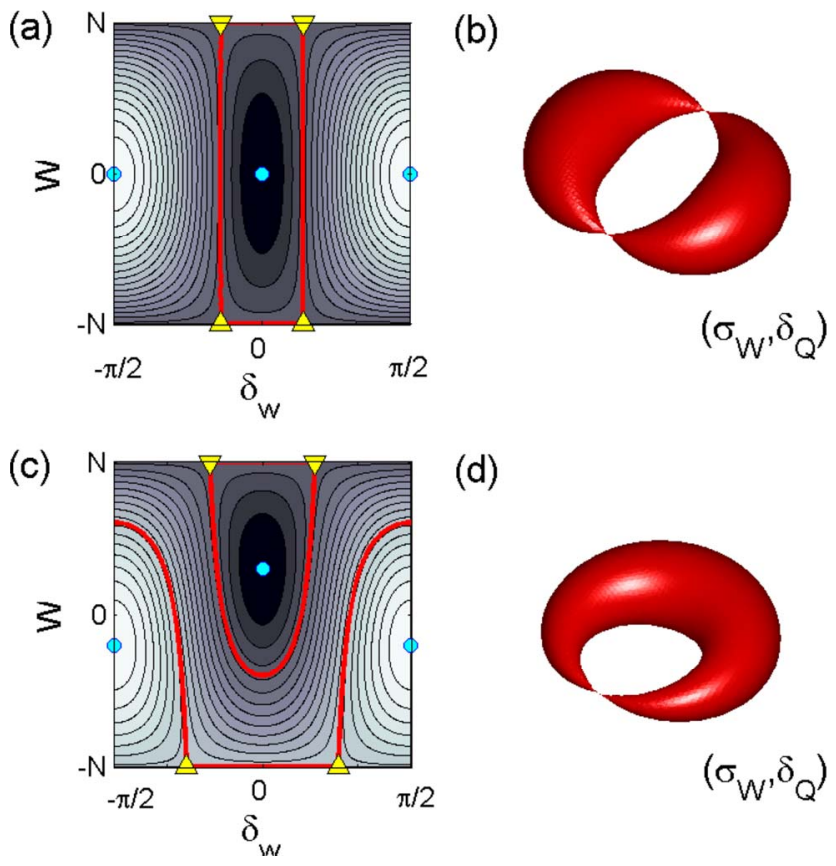

(d)

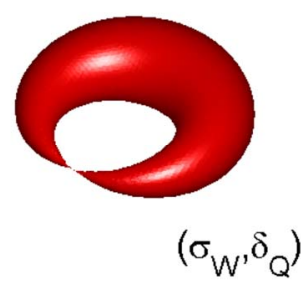

FIG. 7. (Color online) For some $\mathbf{F}$ and $\mathbf{B}$ there exist components of $\left(Q, h_{2}\right)$ level sets that are pinched 2-tori at $Q=0$. (a) Contours of constant $h_{2}$ on the unrolled cylinder representation of the $\left(W, \delta_{W}\right)$ surface for $Q=0, \chi=0$, and $\theta_{1}<\theta<\theta_{2}$. A particular contour for which $\partial h_{2} / \partial W=0$ on each of its points is highlighted (red online). This level set meets the north and south poles $(W= \pm N)$ at $T$ points which are marked with (yellow online) triangles. All points $\left(W, \delta_{W}\right)$ for which $|W|<N$ are crossed by a circle having the coordinate $\delta_{Q}$. However, at the poles $W= \pm N$, no such circle exists. (b) Therefore the corresponding level set has the topology of a doubly pinched torus. Everywhere except at the poles it is a smooth twodimensional surface. Coordinates on the two-dimensional sections of this surface are $\left(\sigma_{W}, \delta_{Q}\right)$, where $\sigma_{W}$ is a coordinate around the contour on the $\left(W, \delta_{W}\right)$ surface. (c) These contours are obtained from contours similar to those of (a) by tilting the fields from perpendicular such that $\chi / \omega_{f} N^{3}=0.2$. The contour that passed through both poles at perpendicular fields divides into the two highlighted (red online) contours at different energies for nonzero $\chi$. (d) The doubly pinched torus in perpendicular fields is split into two singly pinched tori at different energies when the fields are tilted. 
Fig. 4, it can be seen that for $0<|W|<N$ the $\left(Q, \sigma_{Q}\right)$ surface is a cylinder which is capped by rings of $\psi_{1}$ and $\psi_{2}$ at either end, while at $W=0$ it is connected like a sphere.

The intersection of a $\left(Q, h_{2}\right)$ torus with surface of section (i) is a ring with coordinate $\sigma_{Q}$, and the integral of Eq. (28) over a surface bounded by the Pauli orbit may be taken to be [33]

$$
J_{Q}\left(Q, h_{2}\right)=Q .
$$

Thus, $Q$ is simultaneously a conserved quantity, a primitive action, and a locally smooth action variable near every torus.

The definition of the other action is complicated because the $\left(W, \delta_{W}\right)$ surface is connected like a sphere, and because the area of that surface is not a smooth function of $Q$ near $Q=0$.

Consider a level set of the fully reduced Hamiltonian on the $\left(W, \delta_{W}\right)$ surface defined by the equation $h_{2}=E_{2}$. The level set may consist of a single connected component, or multiple disjoint components on the surface. Each disjoint component may or may not contain a relative fixed point, where Eqs. (40) vanish. If a disjoint component does not contain a fixed point and does not pass through the north or south pole at $Q=0$, it forms a smooth one-dimensional closed curve on the $\left(W, \delta_{W}\right)$ surface [34]. Each such curve (i) divides the $\left(W, \delta_{W}\right)$ surface into two areas, and (ii) forms a corresponding contour on the unrolled cylinder which is either an apparent librator or an apparent rotator (Fig. 8). The two areas created on the cylinder by such a contour must sum to the total $\left(W, \delta_{W}\right)$ surface area $2 \pi(N-|Q|)$, and are identified with the areas inside and outside the contour of an apparent librator, or above and below the contour of an apparent rotator.

The classical action integral is defined for each apparent rotator or librator and is directly proportional to the canonical area $d W \wedge d \delta_{W}$ which is bounded by the level curve of $h_{2}$ on the $\left(W, \delta_{W}\right)$ surface. The topology of this surface implies that there are two equally valid areas, which in general have different values. Thus we define for each disjoint smooth curve of the level set $h_{2}=E_{2}$ two equally valid action integrals which we refer to as primitive actions [35]:

$$
\begin{aligned}
& J_{+}\left(h_{2} ; N, Q\right)=\frac{1}{2 \pi} \int_{A_{+}\left(h_{2}\right)} d W \wedge d \delta_{W}, \\
& J_{-}\left(h_{2} ; N, Q\right)=\frac{1}{2 \pi} \int_{A_{-}\left(h_{2}\right)} d W \wedge d \delta_{W} .
\end{aligned}
$$

The integral is over the area of the $\left(W, \delta_{W}\right)$ surface that is bounded by the curve of constant $h_{2}$, and the + and - subscripts have the following meaning. Each apparent rotator or librator is a contour which bisects the total phase space into two areas $A_{1}$ and $A_{2}$, such that $A_{1}+A_{2}=2 \pi(N-|Q|)$ (Fig. 6). Then, the functions in Eqs. (44) could have been defined as $J_{1}\left(h_{2}\right)=A_{1} / 2 \pi$ and $J_{2}\left(h_{2}\right)=A_{2} / 2 \pi$. However, since the sum of the areas is fixed, and no two level sets can intersect, one of the two functions $J_{1}, J_{2}$ is an increasing function of $h_{2}$ while the other is a decreasing function. We denote the increasing function $J_{+}\left(h_{2}\right)=A_{+} / 2 \pi$ and the decreasing function $J_{-}\left(h_{2}\right)=A_{-} / 2 \pi$.
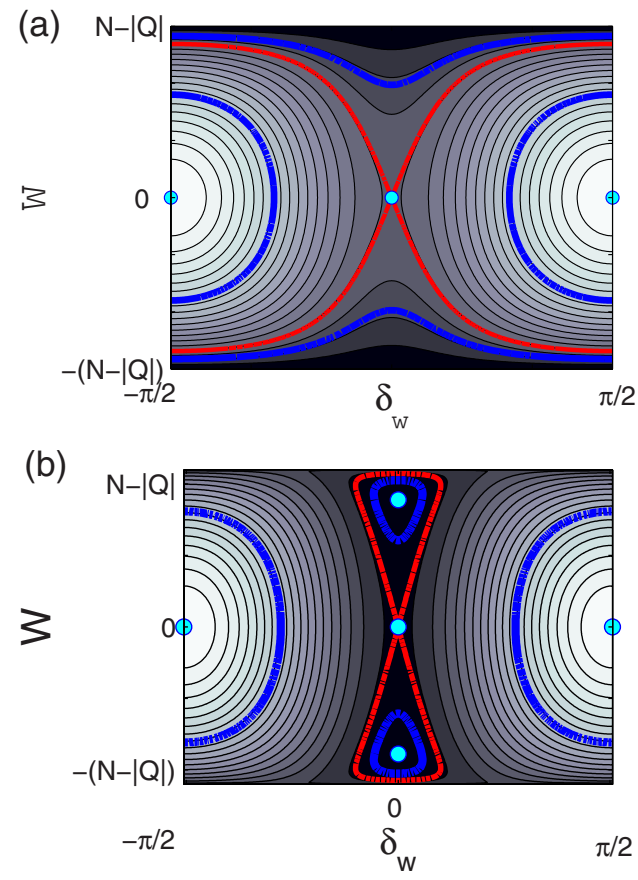

FIG. 8. (Color online) Contours of the Hamiltonian $h_{2}$ on the unrolled cylinder representation of the $\left(W, \delta_{W}\right)$ surface at $\theta=5^{\circ}, N$ $=15, \chi=0$, and (a) $Q=0$ and (b) $Q=4$. The surface has the topology of a sphere such that left and right edges are identified, and the top and bottom edges each consist of a single point. Darker regions indicate lower energies, points indicate fixed points, and an x-point separatrix is marked (red online) as are three other disjoint contours (blue online). At $Q=0$ in (a), fixed points are present, but not marked at $W= \pm N$. As $Q$ increases to $Q=4$ in (b), these o points migrate from the poles toward the equator $(W=0)$ along $\delta_{W}=0$, to form the two additional o points marked in (b). Each disjoint contour of $h_{2}$ which does not contain a fixed point forms either an apparent rotator or an apparent librator, bisecting the total phase space into two areas $A_{1}$ and $A_{2}$, such that $A_{1}+A_{2}=2 \pi(N-|Q|)$. An appropriate action is defined by either $J_{1}\left(h_{2}\right)=A_{1} / 2 \pi$ or $J_{2}\left(h_{2}\right)$ $=A_{2} / 2 \pi$. We identify the $J$ that increases with energy as $J_{+}$, and the one that decreases with energy as $J_{-}$. For each energy greater than that of the separatrix, there is a single apparent librator, localized about the maximal energy o point at $W=0, \delta_{W}= \pm \pi / 2$. Each librator admits the construction of two actions. If $A_{1}$ is the area containing the maximal o point, then $J_{-}=A_{1} / 2 \pi$ is a strictly decreasing function of energy, while $J_{+}=A_{2} / 2 \pi$ is strictly increasing. For all energies below that of the separatrix, there are two disjoint contours having the same value of $h_{2}$ (a classical double degeneracy). One contour $C_{N}$ is localized in the "north" $(W>0)$, while the other $C_{S}$ is in the "south" $(W<0)$. In such a case, both $J_{+}\left(h_{2}\right)$ and $J_{-}\left(h_{2}\right)$ are defined for each disjoint contour. For $C_{N}$, let $A_{2}$ be the area containing the minimal energy o point [which is located at (a) the north pole for $Q=0$, and (b) $W \approx 9, \delta_{W}=0$ for $\left.Q=4\right]$. Then $J_{+}^{(N)}=A_{2} / 2 \pi$ is a strictly increasing function of $h_{2}$, while $J_{-}^{(N)}=A_{1} / 2 \pi$ is strictly decreasing. Similarly for $C_{S}$, if $A_{2}$ contains the minimal energy at or near the south pole, $J_{+}^{(S)}=A_{2} / 2 \pi$, while $J_{-}^{(S)}=A_{1} / 2 \pi$.

As was previously mentioned, for some values of $h_{2}$, there may be multiple contours which are mutually disjoint, but have the same constant value $h_{2}=E_{2}$ (Fig. 6). Since each of the individual contours $C_{i}$ that does not contain a fixed point bisects the $\left(W, \delta_{W}\right)$ surface, it follows that for every 
such contour $C_{i}$ there exists a pair of functions $J_{+}^{(i)}$ and $J_{-}^{(i)}$, given by Eqs. (44).

It follows directly from the above discussion that $J_{+}$and $J_{-}$cannot both be smooth functions of $Q$ near $Q=0$. Since the total canonical area on $\Gamma_{N, Q}$ is $\pi 2(N-|Q|)$, by their definitions [Eqs. (44)]

$$
J_{+}+J_{-}=N-|Q| .
$$

The derivative with respect to $Q$ of the right-hand side of Eq. (45) is not continuous at $Q=0$. Therefore either $J_{+}$, or $J_{-}$, or both must have a discontinuous derivative with respect to $Q$ at $Q=0$.

In Fig. 9 we consider a classical spectrum for nearly perpendicular fields. Each point of the dashed red boundary represents a 1-torus. The (cyan online) diamonds each represent a singly pinched torus. All points inside the (red online) dashed boundary, but excluding the two points marked by the (cyan online) diamonds, represent a 2-torus. From each 2-torus we may construct the primitive actions $J_{+}$and $J_{-}$and plot their contours. In Fig. 9(a) the contours of $J_{+}\left(Q, h_{2}\right)$ are smooth for all points on the spectrum except along $Q=0$, where they are smooth only for energies less than the energy of the lower diamond. At energies above the lower diamond, $\partial J_{+} / \partial Q$ has a jump discontinuity at $Q=0$. The magnitude of the jump is greater above the upper diamond. In Fig. 9(b) we plot the contours of $J_{-}\left(Q, h_{2}\right)$, and find an analogous situation. Here the contours are smooth at energies higher than the upper diamond, and the magnitude of the jump discontinuity increases as we pass each diamond to lower energies.

We can understand the behavior of such discontinuities quantitatively by examining the area generated by level curves of $h_{2}$ on a family of schematic cylinders parametrized by $Q$ (Fig. 10).

Consider a family of level sets with a fixed value of $h_{2}$, ranging over an infinitesimal interval of $Q$, centered about $Q=0$. This family will intersect $\Gamma_{N, Q}$ at each $Q$ of the infinitesimal interval, forming a contour on its cylindrical representation (Fig. 10). If no member of this family contains a fixed point, or passes through $W= \pm N$, then either all members of the family form apparent rotators, or all members form apparent librators on the cylindrical representation of the $\Gamma_{N, Q}$.

Each apparent rotator [Fig. 10(a)] has one associated area that contains one pole, and a complementary area containing the other pole. Since the coordinates on the cylinder are $W$ with $-(N-|Q|) \leq W \leq(N-|Q|)$, and $\delta_{W}$ with $-\pi / 2 \leq \delta_{W}$ $<\pi / 2$, the complementary areas are

$$
\begin{aligned}
& A_{\mathrm{rot}}^{\mathrm{above}}=\pi(N-|Q|)-\int_{-\pi / 2}^{\pi / 2} W d \delta_{W}=2 \pi J_{\mathrm{rot}}^{\mathrm{above}}, \\
& A_{\mathrm{rot}}^{\mathrm{below}}=\pi(N-|Q|)+\int_{-\pi / 2}^{\pi / 2} W d \delta_{W}=2 \pi J_{\mathrm{rot}}^{\mathrm{below}} .
\end{aligned}
$$

The value of the integral $I=\int W\left(h_{2} ; Q\right) d \delta_{W}$ is a smooth function of $Q$ provided that the level set of $h_{2}$ does not contain a fixed point. It follows that, for a family of apparent rotators, both $\partial J_{+} / \partial Q$ and $\partial J_{-} / \partial Q$ include a term equal to $-\frac{1}{2} \operatorname{sgn}(Q)$, and therefore are discontinuous at $Q=0$.
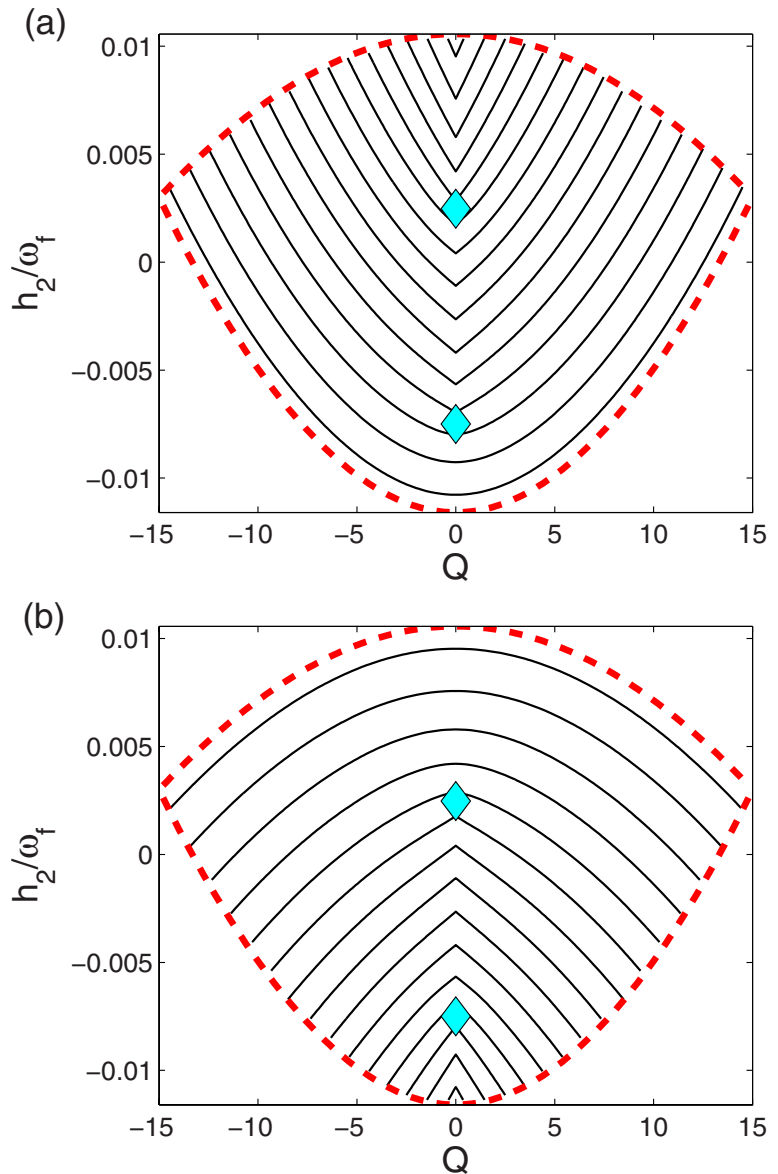

FIG. 9. (Color online) Contours of the primitive actions are plotted on a classical spectrum in near perpendicular fields with $N$ $=15, \theta=50^{\circ}, \omega_{f}=10^{-6}, \chi / \omega_{f} N^{3}=0.2$. All points inside the dashed red boundary and excluding the points marked by the (cyan online) diamonds represent a 2 -torus with coordinates $\left(\sigma_{Q}, \sigma_{W}\right)$. The $\Gamma_{N, Q}$ located at $Q=0$ is plotted in Fig. 7(c), and we identify each of the (cyan online) diamonds with a singly pinched torus. The primitive actions $J_{+}\left(Q, h_{2}\right)$ and $J_{-}\left(Q, h_{2}\right)$ are defined on each 2-torus but may not be smooth at $Q=0$. (a) $\partial J_{+} / \partial Q$ is continuous across $Q=0$ at energies less than that of the low-energy singly pinched torus. But at all greater energies, $\partial J_{+} / \partial Q$ has a jump discontinuity. (b) $\partial J_{-} / \partial Q$ is continuous across $Q=0$ at energies greater than that of the highenergy singly pinched torus, but has a jump discontinuity at all lower energies. Such structure in the primitive actions can be quantitatively determined for any field parameters by examining the behavior of the joint level sets of $\left(Q, h_{2}\right)$ at fixed $h_{2}$ in a neighborhood of $Q$ centered about $Q=0$.

Each apparent librator [Fig. 10(b)] has one associated area that contains neither the north nor the south pole, while the complementary area contains both:

$$
\begin{gathered}
A_{\text {lib }}^{\text {inside }}=\oint W d \delta_{W}=2 \pi J_{\text {lib }}^{\text {inside }}, \\
A_{\text {lib }}^{\text {outside }}=2 \pi(N-|Q|)-\oint W d \delta_{W}=2 \pi J_{\text {lib }}^{\text {outside }} .
\end{gathered}
$$

Again the integral is smooth provided the level set does not contain a fixed point, and it follows that the primitive action 
(a)

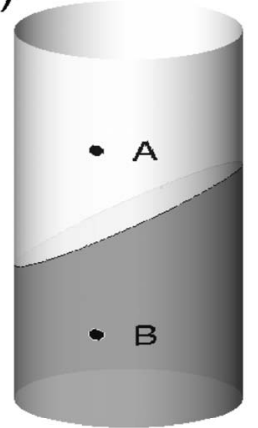

(b)

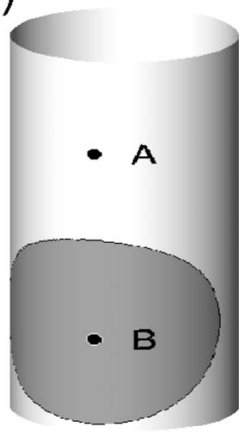

FIG. 10. Most level sets of the second-order Hamiltonian $h_{2}$ form apparent rotators or apparent librators on a schematic cylinder representation of a $\left(W, \delta_{W}\right)$ surface, parametrized by $Q$. Two points marked $A$ and $B$ schematically represent those o points of $h_{2}$ which attain extremal values on the $\left(W, \delta_{W}\right)$ surface. By interchangeably associating these points with a maximum and minimum, one gains insight into the properties of the actions $J_{+}$and $J_{-}$as functions of $Q$ by investigating the properties of the areas. In general, the contours of $h_{2}$ are smooth curves on the cylindrical surface that are also smooth functions of $Q$. However, the height of the cylinder is $2(N-|Q|)$, which has a discontinuous derivative with respect to $Q$ at $Q=0$. (a) The contour of an apparent rotator divides the phase space into two halves. Although the contour itself is a smooth function of $Q$, neither the shaded and unshaded areas are smooth at $Q=0$. Since the coordinate $W$ is zero at the equator of the cylinder, the shaded area is $\pi(N-|Q|)+\int W d \delta_{W}$, and the unshaded area is $\pi(N-|Q|)$ $-\int W d \delta_{W}$. The value of the integral $I=\int W\left(h_{2} ; Q\right) d \delta_{W}$ is a smooth function of $Q$ provided the level set of $h_{2}$ does not contain a fixed point. Therefore, at $Q=0$, both areas of an apparent rotator have a discontinuity in derivative with respect to $Q$ equal to $-\pi \operatorname{sgn}(Q)$. (b) For an apparent librator, the shaded area is $2 \pi J$, where $J=J_{+}$if $B$ is a minimum, and $J=J_{-}$if $B$ is a maximum. This area is a smooth function of $Q$. The unshaded area is equal to $2 \pi(N-|Q|-J)$ and is composed of a part that is smooth and a part that has a discontinuity in slope equal to $-2 \pi \operatorname{sgn}(Q)$ at $Q=0$. Using only these observations and a map of the contours of $h_{2}$ at $Q=0$, one may determine the discontinuities that appear in $J_{+}$and $J_{-}$for all parameters.

associated with the area inside the apparent librator has a smooth derivative with respect to $Q$ at $Q=0$, while $\partial J^{\text {outside }} / \partial Q$ contains a term equal to $-\operatorname{sgn}(Q)$ and is therefore discontinuous at $Q=0$. If a family of librators encircles a minimum of $h_{2}, J_{+}$will pass smoothly through $Q=0$, while if the family encircles a maximum, $J_{-}$will be smooth.

Thus we conclude the following. (i) $\partial J_{+} / \partial Q$ has a jump discontinuity at $Q=0$ described by a term $-\frac{1}{2} \operatorname{sgn}(Q)$ for each pole the associated area contains. (ii) For apparent rotators both the $J_{+}$and $J_{-}$areas contain one pole. (iii) For apparent librators encircling a maximum, the $J_{-}$area does not contain any pole while the $J_{+}$area contains both. (iv) For apparent librators encircling a minimum, the $J_{+}$area does not contain any pole while the $J_{-}$area contains both. We can summarize these conclusions compactly by

$$
\frac{\partial J_{ \pm}}{\partial Q}=-\frac{z_{ \pm}}{2} \operatorname{sgn}(Q)+(\text { smooth }),
$$

where

$$
\begin{aligned}
& z_{+}= \begin{cases}1, & \text { rotators, } \\
0, & \text { librators encircling a minimum, } \\
2, & \text { librators encircling a maximum, }\end{cases} \\
& z_{-}= \begin{cases}1, & \text { rotators, } \\
2, & \text { librators encircling a minimum, } \\
0, & \text { librators encircling a maximum }\end{cases}
\end{aligned}
$$

The type of apparent family present near $Q=0$ depends on $\mathbf{F}$ and $\mathbf{B}$ and can be determined for all field parameters by analysis of the fully reduced Hamiltonian.

Let us look back to Figs. 9(a) and 9(b). If we investigate the corresponding contours of the Hamiltonian on $\Gamma_{N, Q}$ [plotted at $Q=0$ in Fig. 7(c)], we find that near $Q=0$ and at energies greater than that of the upper diamond, the level sets form families of apparent librators which contain a maximum of $h_{2}$. At energies less than that of the lower diamond the families form apparent librators containing a minimum. Meanwhile, at energies which lie between the diamonds, there are families of apparent rotators. The patterns in the magnitudes of the discontinuities are explained by Eqs. (48) and (49).

\section{B. Intrinsically multivalued smooth classical action variables}

In order to construct a smooth classical action variable, a discontinuous primitive action must be smoothly continued across $Q=0$. If we interpret the primitive action $J_{ \pm}\left(Q, h_{2}\right)$ as the principal branch of a smooth but intrinsically multivalued action variable, then at each crossing of $Q=0$ at energies for which $z_{ \pm}$is nonzero, a new branch of $J_{ \pm}\left(Q, h_{2}\right)$ is encountered. Each new branch consists of the old branch plus an additional term:

$$
\begin{aligned}
& J_{+}^{\text {continued }}=J_{+}^{\text {old }}+\operatorname{sgn}(Q) z_{+} Q, \\
& J_{-}^{\text {continued }}=J_{-}^{\text {old }}+\operatorname{sgn}(Q) z_{-} Q,
\end{aligned}
$$

where $z_{ \pm}$is given by Eqs. (49). The additional terms in the new branches repair the intrinsically discontinuous slope at $Q=0$ [Eq. (48)] but may introduce a multivalued smooth classical action variable if a single sheet cannot be used to cover an entire spectrum. In Fig. 11 the principal action $J_{+}$is continued across $Q=0$ into $Q<0$ at energies which have two different values of $z_{+}$. In both cases it is impossible to cover the entire spectrum with a single-valued and smooth classical action variable.

\section{Monodromy and vector transport}

To classify classical spectra, we consider the smooth transport of two independent vectors tangent to contours of locally smooth action variables. Let $\left(\hat{Q}, \hat{h}_{2}\right)$ be unit vectors in the tangent space of the spectrum. Vectors $\mathbf{t}$ which are tangent to contours of the primitive actions Eqs. (43) and (44) are (with arbitrary orientation convention)

$$
\mathbf{t}_{+}=\frac{\partial J_{+}}{\partial h_{2}} \hat{Q}-\frac{\partial J_{+}}{\partial Q} \hat{h}_{2}
$$


(a)

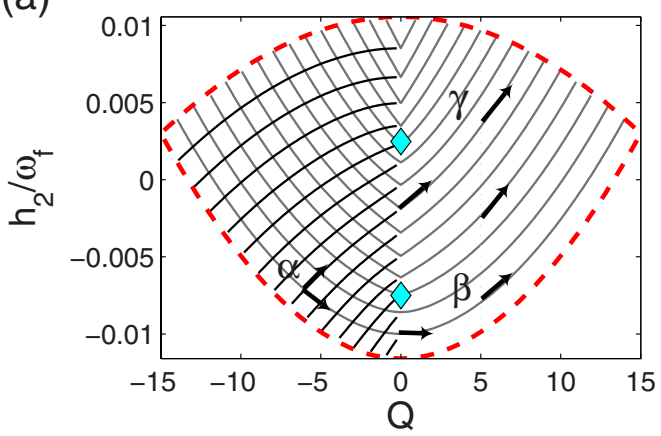

(b)

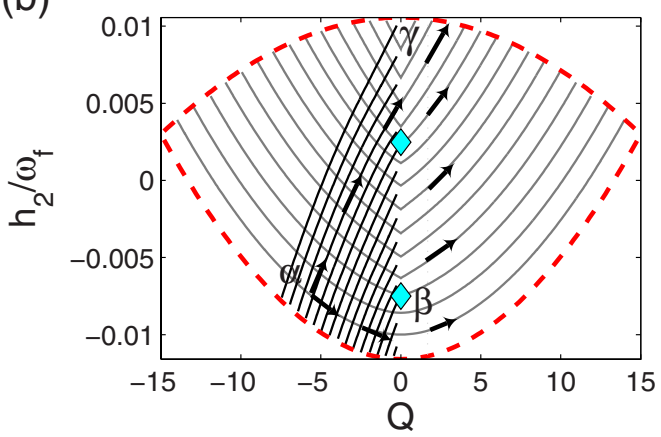

FIG. 11. (Color online) Contours of the primitive action $J_{+}\left(Q, h_{2}\right)$ and its smooth continuation into negative $Q$ are plotted on the classical spectrum. $N=15, \theta=50^{\circ}, \omega_{f}=10^{-6}, \chi / \omega_{f} N^{3}=0.2$. Lighter contours are of the principal branch, and the additional darker contours are of the first continued branch encountered when smoothly continuing across $Q=0$ into $Q<0$. (a) Here the contours are continued through the rotators where $z_{+}=1$, such that $J_{+}$is smoothly joined to the branch $J_{+}-Q$ at $Q=0$ for energies that lie between the two (cyan online) diamonds. (b) Here the contours are continued through the librators where $z_{+}=2$ such that $J_{+}$is smoothly joined to the branch $J_{+}-2 Q$ at $Q=0$ for energies that lie above the upper (cyan online) diamond. To classify this classical spectrum, consider the smooth transport of vectors tangent to the spectrum about the counterclockwise, closed loops marked $\alpha \beta \gamma$. In both (a) and (b), there is a passage to a second branch at $Q=0$ between $\gamma$ and $\alpha$ such that the vector does not return to itself at $\alpha$. The angle of rotation is greater when the loop encircles both (cyan online) diamonds in (b).

$$
\begin{gathered}
\mathbf{t}_{-}=\frac{\partial J_{-}}{\partial h_{2}} \hat{Q}-\frac{\partial J_{-}}{\partial Q} \hat{h}_{2}, \\
\mathbf{t}_{Q}=\hat{h}_{2} .
\end{gathered}
$$

Vectors which are tangent to contours of the smoothly continued action are given by

$$
\begin{aligned}
& \mathbf{t}_{+}^{\text {continued }}=\frac{\partial J_{+}}{\partial h_{2}} \hat{Q}-\left(\frac{\partial J_{+}}{\partial Q}+\operatorname{sgn}(Q) z_{+}\right) \hat{h}_{2}, \\
& \mathbf{t}_{-}^{\text {continued }}=\frac{\partial J_{-}}{\partial h_{2}} \hat{Q}-\left(\frac{\partial J_{-}}{\partial Q}+\operatorname{sgn}(Q) z_{-}\right) \hat{h}_{2} .
\end{aligned}
$$

The vectors in Eqs. (52) differ from the vectors tangent to the primitive actions [Eqs. (51)] by a vector tangent to contours of constant $Q$ with integer magnitude.

$$
\begin{aligned}
& \mathbf{t}_{+}^{\text {continued }}-\mathbf{t}_{+}=-\operatorname{sgn}(Q) z_{+} \hat{h}_{2}=-\operatorname{sgn}(Q) z_{+} \mathbf{t}_{Q}, \\
& \mathbf{t}_{-}^{\text {continued }}-\mathbf{t}_{-}=-\operatorname{sgn}(Q) z_{-} \hat{h}_{2}=-\operatorname{sgn}(Q) z_{-} \mathbf{t}_{Q} .
\end{aligned}
$$

These equations imply that, if a primitive action $J_{ \pm}$is smoothly continued across $Q=0$, a vector tangent to its continued contours is rotated with respect to a vector tangent to the primitive $J_{ \pm}$contours at the same point. The transformation between the vectors tangent to the primitive contours $Q$ and $J_{ \pm}$, and vectors tangent to the continued contours can be written in matrix form:

$$
\left[\begin{array}{l}
\mathbf{t}_{Q}^{\text {continued }} \\
\mathbf{t}_{ \pm}^{\text {continued }}
\end{array}\right]=\left[\begin{array}{cc}
1 & 0 \\
-z_{ \pm} \operatorname{sgn}(Q) & 1
\end{array}\right]\left[\begin{array}{l}
\mathbf{t}_{Q} \\
\mathbf{t}_{ \pm}
\end{array}\right]
$$

Looking back to Fig. 11, the consequences of Eq. (54) for smooth transport of vectors about a closed loop is illustrated by a series of arrows. At point $\alpha$ in Fig. 11(a), the vector $\mathbf{v}_{2}=\mathbf{t}_{+}$is tangent to contours of $J_{+}$and is transported along that contour to point $\beta$. Then this vector is transported along the contour $Q=5$ to $\gamma$. To smoothly transport back to $\alpha$, we must pass to the first continued branch of the multivalued action at $Q=0$. As a result, when $\mathbf{v}_{2}$ returns to point $\alpha$ it has been transformed. In contrast, if we transport the vector $\mathbf{v}_{1}$ $=\mathbf{t}_{Q}$ about the same closed loop, $\mathbf{v}_{1}$ returns identically to itself. Using Eq. (54) the transformation of the vectors $\mathbf{v}_{1}$ and $\mathbf{v}_{2}$ is equivalent to multiplication by the matrix $M_{1}$ $=[1,0 ; 1,1]$ written in the basis $\left\{\mathbf{v}_{1}, \mathbf{v}_{2}\right\}$ at $\alpha$ :

$$
M_{1}=\left[\begin{array}{ll}
1 & 0 \\
1 & 1
\end{array}\right], \quad\left\{\mathbf{v}_{1}, \mathbf{v}_{2}\right\}=\left\{\left[\begin{array}{l}
1 \\
0
\end{array}\right],\left[\begin{array}{l}
0 \\
1
\end{array}\right]\right\} .
$$

Such a transformation occurs for transport about any counterclockwise closed loop encircling the lower (cyan online) diamond. Similar considerations applied to the closed loop $\alpha \beta \gamma$ in Fig. 11(b) yield a transformation equivalent to multiplication by the matrix $M_{2}$ in the same basis, where

$$
M_{2}=\left[\begin{array}{ll}
1 & 0 \\
2 & 1
\end{array}\right] .
$$

To fully classify this spectrum we consider all possible closed loops. Similar analysis applied to counterclockwise loops encircling the upper diamond yields Eq. (55) again. Any clockwise traversal of a loop encircling a single diamond yields the matrix inverse to $M_{1}, M_{1}^{-1}=M_{-1}=[1,0$; $-1,0]$, while any encircling both diamonds will yield $M_{2}^{-1}$. Finally, transport about any loop that begins and ends on the same branch will result in a transformation described by the identity matrix $E=[1,0 ; 0,1]$. This exhausts all possible closed loops for any spectrum that is topologically equivalent to that in Fig. 11, as any possible loop is equivalent to some combination of these basis loops. Thus any spectrum having two singly pinched tori at different energies is classified by listing the three matrices $\left\{M_{1}, M_{2}, E\right\}$.

In this manner, spectra for any weak, nearly perpendicular $\mathbf{F}$ and $\mathbf{B}$ (such that $|\chi| \leqslant \omega_{f} N^{3} \ll 1$ ) are classified by a list of matrices which describe the effects of smooth vector transport about all possible closed loops on the spectrum. By Eq. (54) the values of the components of the matrices are deter- 


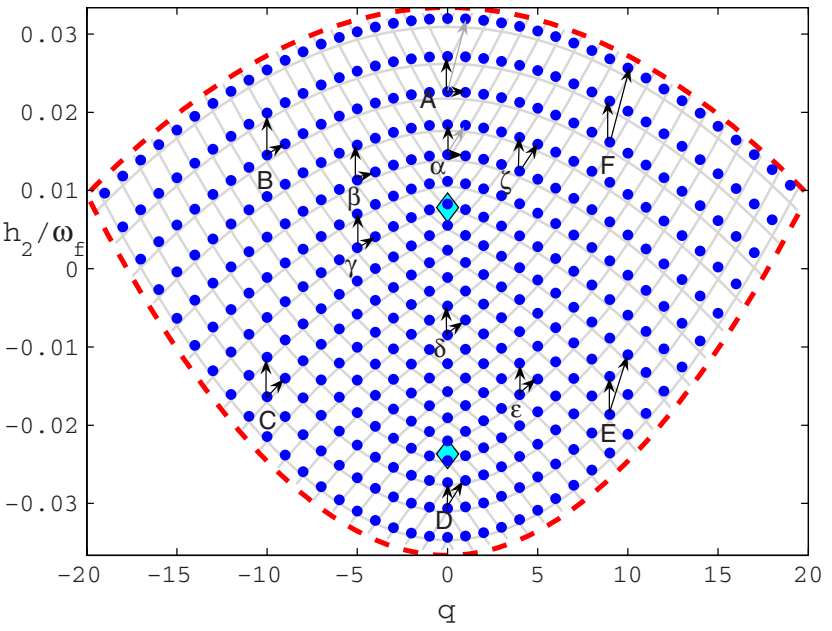

FIG. 12. (Color online) Type-II spectrum. The spectrum $h_{2}$ vs $q$ for $\chi / \omega_{f} n^{3}=0.2, n=20, F=146 \mathrm{~V} / \mathrm{cm}, B=0.302 \mathrm{~T}$, or $\theta=50^{\circ}$ and $\omega_{f}=10^{-6}$. The quantum basis included $n$-manifolds from 18 to 22 . The (blue online) circular dots are derived from quantum calculations, while all other structures are derived from classical perturbation theory and semiclassical quantization of actions. Two independent monodromy circuits are marked by sequences of letters. A $[1,0 ; 2,1]$ defect is characterized by lattice vector transport of $\left\{\mathbf{v}_{1}, \mathbf{v}_{2}\right\}$ along locally smooth action contours connecting points marked by the latin letter cycle $A B C D E F A$. This circuit encircles both point defects and after a complete circuit has been made, $\mathbf{v}_{1}$ returns to the vector $\mathbf{v}_{1}+2 \mathbf{v}_{2}$ depicted in gray at point $A$. A $[1,0 ; 1,1]$ defect is characterized by transport of $\left\{\mathbf{v}_{1}, \mathbf{v}_{2}\right\}$ along smooth contours connecting points marked by the sequence of greek letters $\alpha \beta \gamma \delta \epsilon \zeta \alpha$. When a complete circuit has been made $\mathbf{v}_{1}$ returns to the vector $\mathbf{v}_{1}+\mathbf{v}_{2}$ depicted in gray at point $\alpha$.

mined by the structure of the Hamiltonian $h_{2}$ on $\Gamma_{N, Q}$ in an infinitesimal neighborhood near $Q=0$ through Eqs. (49).

As an alternative to smooth continuation of the primitive actions, one may transform between any overlapping smooth action functions. For example, when continuing around a 2-defect in region II or the $\perp$ M.Int interval, it is easiest to visualize the transport of tangent vectors along the smooth contours $J_{+}$below the defect point, and along contours of $J_{-}$ above (see Fig. 12).

\section{MULTIVALUED ACTIONS AND QUANTUM LATTICE DEFECTS}

The connection between the classical system and quantum calculations is through the EBKM quantization of the actions. In this section, we will see how each resulting semiclassical "state" is associated with quantized values of the constants of the motion, and an associated point in a classical spectrum. A collection of such points forms a lattice of points in the spectrum. When a classical spectrum has an intrinsically multivalued action, the associated lattice has a defect. In this section we also consider the appropriate quantum operators having expectation values which correspond with the quantized constants of the motion. Through this connection, quantum spectra in near perpendicular fields are classified by the presence of monodromy.

\section{A. EBKM quantization}

Quantization of primitive classical actions selects those classical tori which correspond to approximate quantum energy eigenvalues. The semiclassical quantization conditions are

$$
\begin{gathered}
N=n=1,2,3, \ldots, \\
Q=q=-(n-1),-(n-2), \ldots,(n-1) .
\end{gathered}
$$

Comparing semiclassical with calculated quantum spectra, we find that either of the primitive actions $J_{+}$or $J_{-}$is quantized as half integers:

$$
J_{ \pm}=k+\frac{1}{2}, \quad k=0,1, \ldots, n-|q|-1 .
$$

Thus, in a primitive semiclassical approximation, a quantum state is associated with each contour of $h_{2}$ with $J_{+}$or $J_{-}$ quantized as in Eq. (57c) on each $\Gamma_{N, Q}$ at quantized values of $N$ and $Q$ as in Eqs. (57a) and (57b) such that $\Gamma_{N, Q}=\Gamma_{n, q}$. As an example, consider the case $\mathbf{B}=\mathbf{0}$ and $\mathbf{F}$ parallel to the $x$ axis where Eq. (39) is reduced to

$$
h_{\text {Stark }}=-\frac{1}{2 N^{2}}+\frac{3 N F}{2} Q-\frac{N^{4} F^{2}}{16}\left(17 N^{2}-3 Q^{2}-9 W^{2}\right) \text {. }
$$

This Hamiltonian is independent of $\delta_{W}$ such that $W$ is a constant. Then it follows from definitions (44a) and (44b) that, for $B=0, J_{+}^{(N)}=J_{+}^{(S)}=(N-|Q|+|W|) / 2$ and $J_{-}^{(N)}=J_{-}^{(S)}=(N-|Q|$ $-|W|) / 2$. Either of these equations and the EBKM quantization conditions Eqs. (57a) $-(57 \mathrm{c})$ imply $N=n, Q=q$, and $W^{2}$ $=(2 k+1-n+|q|)^{2}$, where $k=0,1,2, \ldots, n-|q|-1$. Subsequent substitution into the Hamiltonian $h_{\text {Stark }}$ above yields an EBKM spectrum which differs from the well-known formulas [36] only by a quantum correction $19 N^{4} F^{2} / 16$.

\section{B. Quantum operators}

To obtain a collection of quantum spectra for the crossed fields Hamiltonian Eq. (2), we expand the wave function in a basis [37] of bound spherical eigenstates $|n, l, m\rangle$ of the unperturbed hydrogen Hamiltonian $\hat{H}_{0}$, and compute the eigenvalues $E_{j}$ and eigenvectors $\left|\Psi_{j}\right\rangle$ of the resulting matrix. There is good agreement between the obtained quantum eigenvalues and the primitive semiclassical energy eigenvalues for almost all states in weak near-perpendicular spectra.

On a finite basis of bound eigenstates of $\hat{H}_{0}$, the matrix $\hat{H}_{0}$ possesses a well-defined inverse $\hat{H}_{0}^{-1}$ such that $\hat{H}_{0} \hat{H}_{0}^{-1}$ $=\hat{H}_{0}^{-1} \hat{H}_{0}=I$. The eigenvalues of $\hat{H}_{0}$ in the $|n, l, m\rangle$ bases are $E_{n}=-\left(2 n^{2}\right)^{-1}$, and it follows that $\hat{H}_{0}^{-1}|n, l, m\rangle=-2 n^{2}|n, l, m\rangle$. The existence of the inverse operator to $\hat{H}_{0}$ in the basis of bound states allows the definition of two additional operators which commute with each other and with $\hat{H}_{0}$ but do not commute with the full Hamiltonian [38]. These operators are the quantum analog of the canonical momenta in Eqs. (26a)-(26d) and require the operator analogs of the angular momentum and Runge-Lenz vectors. Classically the RungeLenz vector is defined by 


$$
\mathbf{A}=\mathbf{p} \times \mathbf{L}-\frac{\mathbf{r}}{|\mathbf{r}|}
$$

The corresponding quantum operator must be Hermitian, and was defined by Pauli as [2]

$$
\hat{\mathbf{A}}=\frac{1}{2}(\hat{\mathbf{p}} \times \hat{\mathbf{L}}-\hat{\mathbf{L}} \times \hat{\mathbf{p}})-\frac{\hat{\mathbf{r}}}{|\hat{\mathbf{r}}|} .
$$

By use of $\hat{H}_{0}^{-1}$ one may extend Pauli's analysis to multiple $n$-manifolds by altering an operator he introduced with the replacement $-2 n^{2} \rightarrow \hat{H}_{0}^{-1}$ :

$$
\omega_{f} \hat{Q}=\frac{B}{2} \hat{L}_{z}+\frac{3 F_{x}}{4} \hat{H}_{0}^{-1} \hat{A}_{x}
$$

and we also define a related operator

$$
\omega_{f} \hat{W}=\frac{B}{2} \sqrt{-\frac{\hat{H}_{0}^{-1}}{2}} \hat{A}_{z}-\frac{3 F_{x}}{2} \sqrt{-\frac{\hat{H}_{0}^{-1}}{2}} \hat{L}_{x} .
$$

From the fundamental canonical commutation relations and definitions (61) and (62) it follows that

$$
\begin{gathered}
{\left[\omega_{f} \hat{Q}, \omega_{f} \hat{W}\right]=0,} \\
{\left[\omega_{f} \hat{Q}, \hat{H}_{0}\right]=0,} \\
{\left[\omega_{f} \hat{W}, \hat{H}_{0}\right]=0 .}
\end{gathered}
$$

Since both operators $(61)$ and (62) commute with $\hat{H}_{0}$, they are diagonal in $n$, with eigenvectors that are linear combinations of the $|n, l, m\rangle$ states at a fixed $n$.

If we define the following function of $n$ :

$$
\omega_{f}(n)=\frac{1}{2} \sqrt{B^{2}+(3 n F)^{2}},
$$

then the eigenvectors of $\omega_{f} \hat{Q}$ [Eq. (61)] have the eigenvalues $\omega_{f}(n) q$ with $q=-(n-1),-(n-2), \ldots,(n-1)$, while the eigenvectors of $\omega_{f} \hat{W}[\mathrm{Eq} .(62)]$ have the eigenvalues $\omega_{f}(n) w$ with $w=-(n-|q|-1),-(n-|q|-2), \ldots,(n-|q|-1)$.

\section{Quantum lattices}

The second- (and higher-)order energy may be defined as

$$
h_{2}^{j}=\left\langle\Psi_{j}|\hat{H}| \Psi_{j}\right\rangle-\left\langle\Psi_{j}\left|\hat{H}_{0}\right| \Psi_{j}\right\rangle-\left\langle\Psi_{j}\left|\omega_{f} \hat{Q}\right| \Psi_{j}\right\rangle .
$$

To scale these expectation values, define

$$
\bar{n}_{j}=\sqrt{-\frac{1}{2}\left\langle\Psi_{j}\left|\hat{H}_{0}^{-1}\right| \Psi_{j}\right\rangle} .
$$

The expectation value in Eq. (65) is scaled by substituting Eq. (66) into Eq. (64) and dividing Eq. (65) by the result,

$$
\frac{h_{2}^{j}}{\omega_{f}}=\frac{1}{\omega_{f}(\bar{n})}\left\langle\Psi_{j}\left|\left(\hat{H}-\hat{H}_{0}-\omega_{f} \hat{Q}\right)\right| \Psi_{j}\right\rangle .
$$

The scaled expectation value of the $\omega_{f} \hat{Q}$ operator is similarly defined as

$$
q_{j}=\frac{1}{\omega_{f}(\bar{n})}\left\langle\Psi_{j}\left|\omega_{f} \hat{Q}\right| \Psi_{j}\right\rangle .
$$

Then a quantum lattice (or energy momentum spectrum or quantum web) of eigenstates of $H$ is constructed as follows. For each eigenstate, which in the limit of zero fields is a member of a specific $n$-manifold, plot $\left(h_{2}\right)_{j}$ [Eq. (65) or Eq. (67)] vs $q_{j}$ [Eq. (68)]. For weak near-perpendicular fields, the result is a lattice consisting of $n^{2}$ points [39]. A quantum lattice appears in Fig. 12. A (blue online) dot is located at the point $\left(h_{2}^{j} / \omega_{f}, q_{j}\right)$ for each quantum state at $n=20$.

The semiclassical approximation to the quantum lattice is constructed by plotting contours of the primitive classical actions $J_{ \pm}$and $Q$ having values that are quantized according to Eqs. (57). Intersections of the contours yield semiclassical eigenvalues. We observe that almost all points in the quantum lattice lie close to such intersections.

The quantum lattices can be classified by considering the transport of lattice vectors along contours of smooth classical action variables (Sec. VI C) and would result in the same list of matrices as the corresponding classical spectrum (Fig. 12). It follows that, if a classical spectrum has a multivalued action variable, the corresponding quantum lattice will have a lattice defect.

\section{STRUCTURE OF SPECTRA IN NEAR- PERPENDICULAR FEILDS}

In this section we finally explain all of the structures in the quantum spectra that were displayed without explanation in Ref. [14]. All structure in those spectra follow from the study of contour plots of $h_{2}$ [Eqs. (35)] on $\Gamma_{N, Q}$, the $\left(W, \delta_{W}\right)$ surface. The following general principles should be recalled. (i) In each region of $(\theta, \chi)$, at each $Q$, the maximum and minimum of $h_{2}$ on $\Gamma_{N, Q}$ give the upper and lower boundaries of the spectrum in the $\left(Q, h_{2}\right)$ plane. (ii) For most values of $\left(Q, h_{2}\right)$ that lie within the spectral boundary, the level sets correspond to either one (classical nondegeneracy) or two (classical double degeneracy) 2-tori in $\Gamma_{N} \sim S^{2} \times S^{2}$. The two fundamental loops of these tori are a Pauli orbit and a connected level contour of $h_{2}$ on $\Gamma_{N, Q}$. (iii) The level sets that are not entirely composed of disjoint tori are marked with either dashed lines (red or magenta online) or (cyan online) diamonds. At $Q=0$, there is no Pauli orbit at the poles of $\Gamma_{N, Q=0}$ such that any contour of $h_{2}$ which passes through a pole forms a pinched torus in $\Gamma_{N}$. At perpendicular fields the energies of the poles are equal and one finds a doubly pinched torus, while in near-perpendicular fields there are two singly pinched tori with different energies. The pinched tori are marked on the spectrum with (cyan online) diamonds. (iii) Discontinuities in primitive actions follow the patterns described in Fig. 10 and are written explicitly in Eqs. (49).

Shown in Fig. 13 is a map of the parameter space $(\chi, \theta)$ for weak near-perpendicular fields with $0 \leq \theta \leq \pi / 2$ and 0 $\leq|\chi| \lesssim \omega_{f} N^{3} \ll 1$. The spectra are divided into regions according to the list of matrices associated with vector transport (Sec. VI C), and the presence of a classical double degeneracy (Sec. V C). As was shown in previous sections, both of these can be determined by analyzing the structure of the fixed points of $h_{2}$ on $\Gamma_{N, Q}$. 


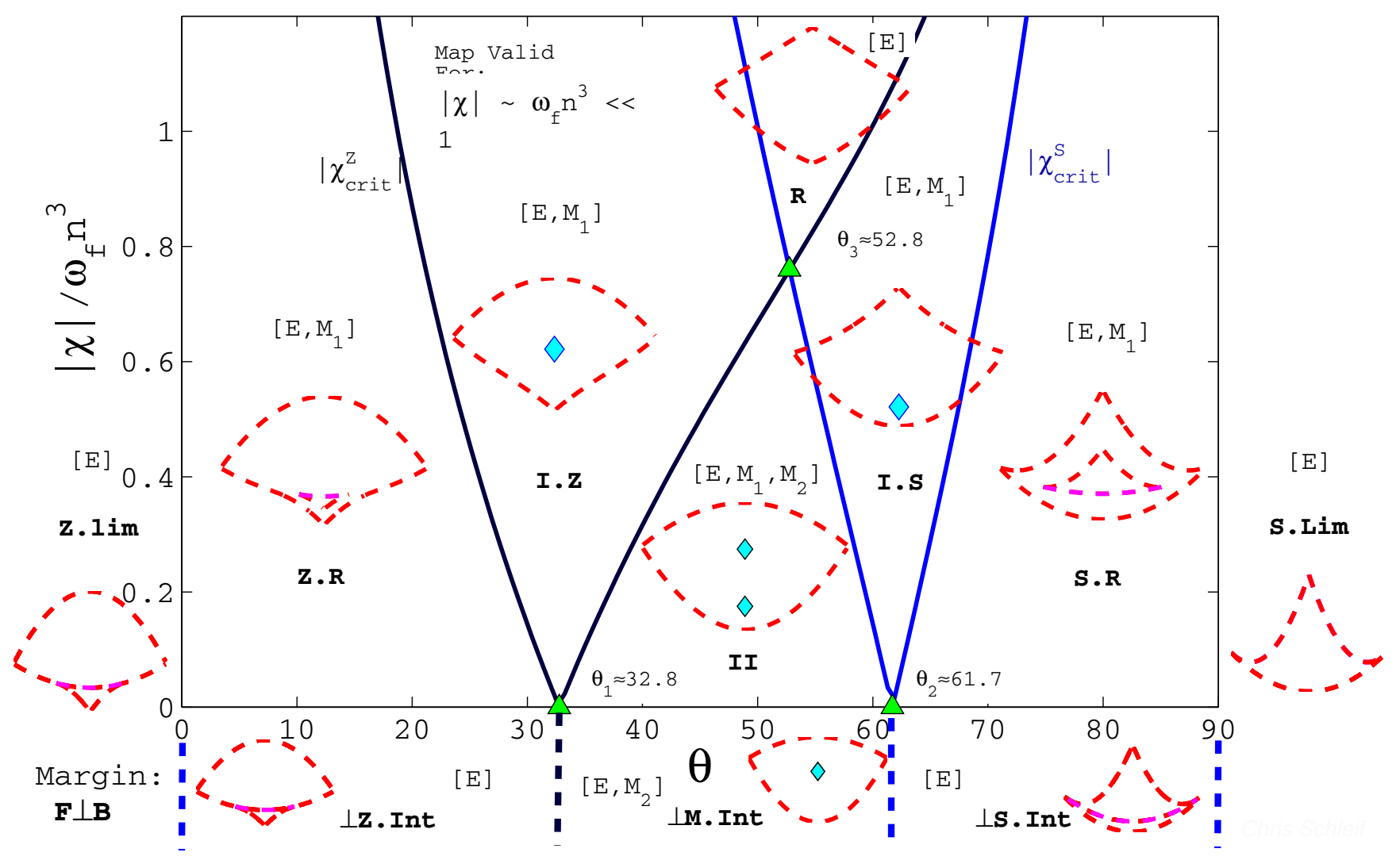

FIG. 13. (Color online) A map of the structure of classical spectra for all $\mathbf{F}$ and $\mathbf{B}$ such that $|\chi| \lesssim \omega_{f} n^{3} \ll 1$. All field magnitude ratios are covered on the horizontal axis by Eq. (36) with $0^{\circ} \leq \theta \leq 90^{\circ}$, with pure magnetic field at $\theta=0^{\circ}$ and pure electric field at $\theta=90^{\circ}$. $\pi / 2-\chi$ is the angle in radians between the electric and magnetic fields, with perpendicular fields $\chi=0$ along the lower margin of the map. For 0 $<|\chi| \lesssim \omega_{f} n^{3} \ll 1$, the (black and blue online) solid lines demarcate six regions. Each region contains spectra that are classified by a list of matrices in square brackets. Plotted in each region approximately above its parameter values are the outlines of representative spectra. Their implied $x$ and $y$ axes are $Q$ and $h_{2}$, respectively; (red online) dashed lines mark the energy of o points of $h_{2}$, (magenta online) dashed lines mark x points, and (cyan online) diamonds indicate the energies of pinched tori. A classical double degeneracy is present in the Stark limit (S.Lim), the perpendicular Stark interval $(\perp$ S.Int), and the Stark region (S.R), as well as near the Zeeman limit in regions marked R, $\perp$ Z.Int, and Z.R. Near the Zeeman limit the degeneracy is contained within the small triangular portions of the spectrum including the lower (red online) boundary. In the Stark limit all points save the lower boundary are doubly degenerate, and the degeneracy in nearby spectra is confined within the small triangular region including its upper (red online) boundary.

Demarcating the different regions of the map are the (black and blue online) solid curves, derived by analysis of $h_{2}$, and given by

$$
\begin{aligned}
& \chi_{\text {crit }}^{Z}(\theta)= \pm \frac{4 N(\alpha-\beta)}{\omega_{f} \sin (2 \theta)}, \\
& \chi_{\text {crit }}^{S}(\theta)= \pm \frac{4 N(\alpha+\beta)}{\omega_{f} \sin (2 \theta)} .
\end{aligned}
$$

Equations (69) divide the near-perpendicular parameter space into six regions, and, when equal to zero, divide the perpendicular field interval $(\chi=0)$ into three subintervals $\theta$ $<\theta_{1}, \theta_{1}<\theta<\theta_{2}$, and $\theta>\theta_{2}$, with

$$
\theta_{1}=\cos ^{-1}\left(2^{-1 / 4}\right) \approx 32.765^{\circ},
$$

$$
\theta_{2}=\cos ^{-1}\left(\sqrt{\frac{\sqrt{6}}{2}-1}\right) \approx 61.701^{\circ} .
$$

There are two critical points where $\chi_{\text {crit }}^{Z}=\chi_{\text {crit }}^{S}$ in weak nearperpendicular fields at

$$
\begin{aligned}
& \chi_{3}= \pm 3^{-1 / 4} \omega_{f} N^{3} \approx \pm 0.7598 \omega_{f} N^{3}, \\
& \theta_{3}=\cos ^{-1}\left(\sqrt{\frac{\sqrt{3}-1}{2}}\right) \approx 52.771^{\circ} .
\end{aligned}
$$

Spectra with $\theta<\theta_{1}$ and $0<|\chi|<\chi_{\text {crit }}^{Z}$ are classically doubly degenerate for all $\left(Q, h_{2}\right)$ that are located in the interior of an inner triangular area. These spectra include the Zeeman limit $(\theta=0, \chi$ meaningless) denoted Z.Lim, the perpendicular Zeeman interval $\left(0<\theta<\theta_{1}, \chi=0\right)$ denoted $\perp$ Z.Int, and the nearperpendicular Zeeman region denoted Z.R. On the map (Fig. 13), the boundaries of these degenerate triangular subareas are marked on the sample spectra plotted above Z.R, Z.Lim, and $\perp$ Z.Int because the boundaries consist of level sets 

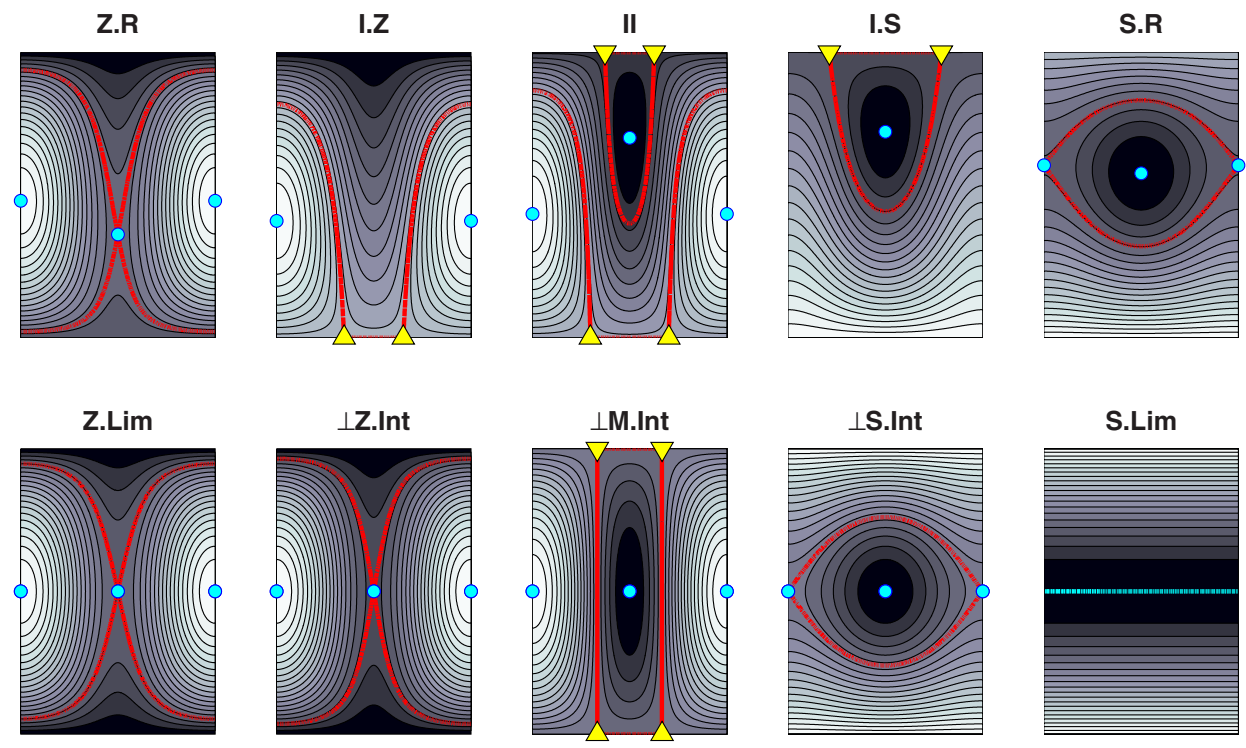

FIG. 14. (Color online) The structure of $\Gamma_{N, Q}$ at $Q=0$ for all field configurations such that $|\chi| \lesssim \omega_{f} n^{3} \ll 1$, except the regular region R. Each $\Gamma_{N, Q=0}$ depicted above is structurally similar to every $\Gamma_{N, Q=0}$ within the same region marked on the map with the same label (Fig. 13). Each region for perpendicular fields $\chi=0$ has a representative in the bottom row. For perpendicular fields (bottom row) all except $\perp$ M.Int have a classical double degeneracy and effective o points at each pole, while $\perp$ M.Int has a doubly pinched torus which passes through both poles. In near-perpendicular fields (top row), there are six parameter regions, five of which have a representative in the top row. Z.R and S.R have effective o points at both poles and families of doubly degenerate apparent rotators, separated from nondegenerate librators by an x-point separatrix. I.S and I.Z have a singly pinched torus at one pole, and an effective o point at the other, while region II has a singly pinched torus at each pole. The region that is not represented is the completely regular region $\mathrm{R}$ where the structure on $\Gamma_{N, Q}$ is composed entirely of apparent rotators with an effective o point at each pole, one maximum and one minimum.

which are not entirely composed of tori. The lower boundary (red dashed line online) of this region is also classically doubly degenerate, but the upper boundary (magenta dashed line online) marks the energy of an x-point separatrix on $\Gamma_{N, Q}$, and is therefore not doubly degenerate.

Spectra with $\theta>\theta_{2}$ and $0<|\chi|<\chi_{\text {crit }}^{S}$ have a similar classically doubly degenerate inner triangular area. These spectra include the Stark limit $(\theta=\pi / 2, \chi$ meaningless $)$ denoted S.Lim, the perpendicular Stark interval $\left(\theta_{2}>\theta>\pi / 2, \chi=0\right)$ denoted $\perp$ S.Int, and the near-perpendicular Stark region denoted S.R. Here, points on the upper boundary are classically doubly degenerate, while the lower boundary (dashed magenta online) marks the energy of an x-point separatrix on $\Gamma_{N, Q}$, and is not degenerate.

To understand how the this map is derived, we consider the structure of $h_{2}$ on the $\Gamma_{N, Q}$ in the various parameter regions.

\section{A. Structure of $h_{2}$ on $\Gamma_{N, Q}$ at $Q=0$}

In Sec. VI we showed how the determination of a list of matrices, used to classify a spectrum, depends on the classification of level sets as apparent rotators or apparent librators on $\Gamma_{N, Q}$ at $\mathrm{Q}=0$. In Fig. 14 we plot a representative $\Gamma_{N, Q=0}$ for each region of the map except the regular region $\mathrm{R}(\mathrm{R}$ has a simple structure which is described in the figure caption). Near-perpendicular regions comprise the top row, and perpendicular regions are on the bottom. For all $\left(\theta, \chi / \omega_{f} N^{3}\right)$ inside a given region as demarcated in Fig. 13, the contours of $h_{2}$ on $\Gamma_{N, Q=0}$ will have a similar structure to the represen- tative plotted in Fig. 14 such that the same number and types of fixed points will be present. [i.e., for all $\left(\theta, \chi / \omega_{f} N^{3}\right)$ inside a given region, the contours of $h_{2}$ on $\Gamma_{N, Q}$ are topologically equivalent].

Nonpolar fixed points are located at the coordinates

$$
\begin{gathered}
\left(W, \delta_{W}\right)=\left(-\frac{\zeta}{2(\beta-\alpha)}, 0\right), \\
\left(W, \delta_{W}\right)=\left(-\frac{\zeta}{2(\beta+\alpha)}, \pm \frac{\pi}{2}\right),
\end{gathered}
$$

provided that the resulting $W$ satisfies the requirement $W$ $<N$. These fixed points are either $\mathrm{x}$ points or o points in accordance with Eq. (42). Additional polar o points are located at both poles of $\Gamma_{N, Q=0}$ in regions $R$, Z.R, S.R, Z.Lim, $\perp$ Z.Int, $\perp$ S.Int, and S.Lim, and at a single pole of $\Gamma_{N, Q=0}$ in regions I.Z and I.S.

For $\Gamma_{N, Q=0}$ in near perpendicular fields, there are two regions (Z.R and S.R) which contain families of rotators that are classically doubly degenerate. In both cases the degenerate rotators are separated from nondegenerate librators by an $\mathrm{x}$-point separatrix. In perpendicular fields all but $\perp$ M.Int have a similar double degeneracy; however, note that the Stark limit does not have a separatrix and is composed almost entirely of doubly degenerate rotators [40]. If we begin at perpendicular fields in $\perp$ Z.Int $(\perp$ S.Int $)$ and increase $\chi$, as we pass through Z.R (S.R) the location of the $\mathrm{x}$ point migrates south (north) reaching the pole at $\chi_{\text {crit }}^{Z}\left(\chi_{\text {crit }}^{S}\right)$ where we 

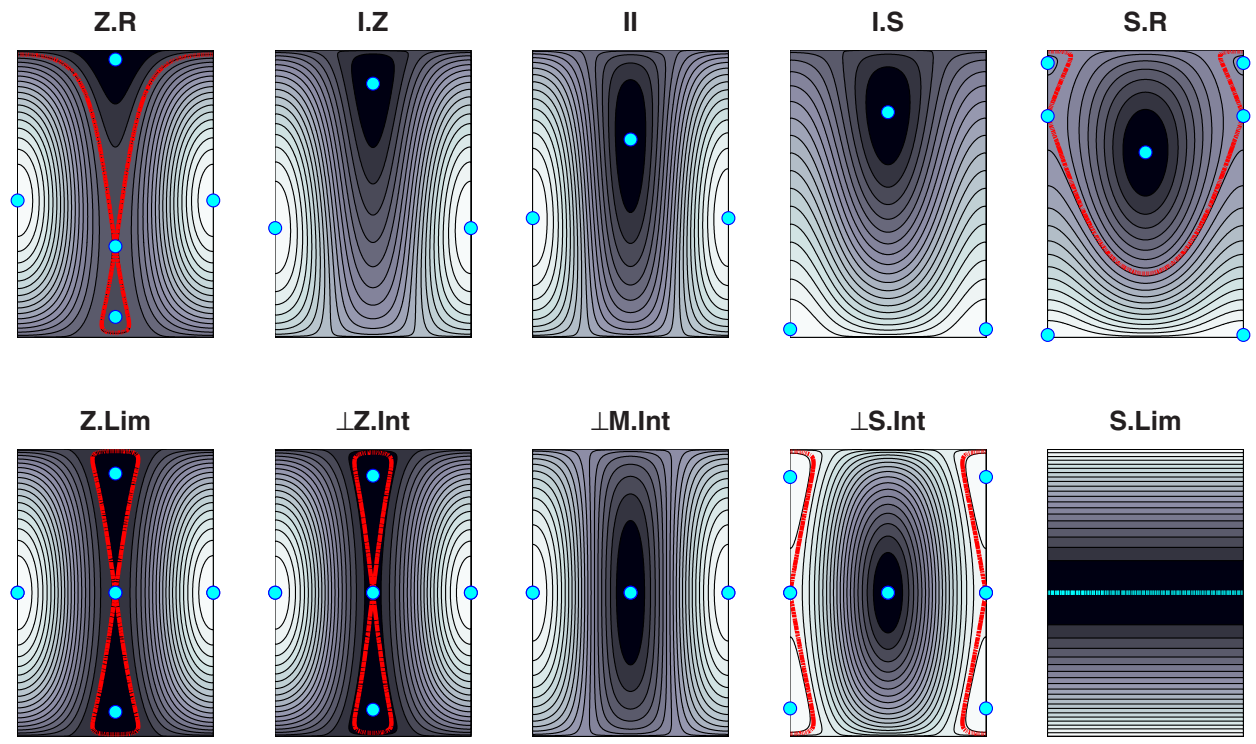

FIG. 15. (Color online) The structure of $\Gamma_{N, Q}$ for $0<Q<N$ (and $0<Q<Q_{\text {crit }}$ in Z.R, Z.Lim, S.R, $\perp$ S.Int) for all field configurations such that $|\chi| \lesssim \omega_{f} n^{3} \ll 1$ except R. As $|Q|$ increases from zero, effective o points at the poles in Fig. 14 migrate toward the equator along either $\delta_{W}=0$ or $\delta_{W}= \pm \pi / 2$ in all relevant regions (and in R) except for the Stark limit (S.Lim). The structure of $\Gamma_{N, Q}$ in the Stark limit is the same for all $Q$. If the region also contains a separatrix dividing degenerate and nondegenerate contours, then there exists a $|Q|=Q$ crit such that a migrating o point will annihilate the $\mathrm{x}$ point on the separatrix, removing both the separatrix and the degeneracy from the structure of the contours. For $0<Q<N$, the $\Gamma_{N, Q}$ in regions $\perp$ M.Int, I.Z, II, I.S, and R all share the same simple structure, determined by a single maximum energy, and a single minimum energy o point for all $0<Q<N$. Such structure is attained in all regions except the Stark limit for $Q$ $>Q_{\text {crit }}$.

pass to I.Z (I.S). During this passage the energy range and phase space area of the southern (northern) rotators shrinks to zero.

In near-perpendicular fields are three regions (I.Z, II, and I.S) which contain level sets associated with singly pinched tori. On $\Gamma_{N, Q}$ these level sets pass through the poles at the $T$-point angles $\delta_{W}^{T \pm}$ :

$$
\delta_{W}^{T \pm}=\frac{1}{2} \cos ^{-1}\left(\frac{\beta}{\alpha} \pm \frac{\zeta}{2 \alpha N}\right) .
$$

In Eq. (73), the + sign yields the angles of $T$ points at the north pole, while the - sign yields the angles of $T$ points at the south pole. Regions I.S and I.Z each have one singly pinched torus with its pinch point located at one pole of $\Gamma_{N, Q=0}$. Region II has two singly pinched tori, one of which has a pinch point located at the north pole of $\Gamma_{N, Q=0}$, and the other a pinch point at the south pole. In perpendicular fields there is one interval $(\perp$ M.Int $)$ which has one doubly pinched torus, with a pinch point located at each pole of $\Gamma_{N, Q=0}$.

\section{B. Structure of $h_{2}$ on $\Gamma_{N, Q}$ for $|Q|>0$}

In Fig. 14 we plot a representative $\Gamma_{N, Q}$ with nonzero $Q$ for each region of the map except the regular region $\mathrm{R}(\mathrm{R}$ has a simple structure that is described in the figure caption). In all regions except the Stark limit, as $|Q|$ is increased from $Q=0$, o points which are present at a pole at $Q=0$ become nonpolar o points and migrate toward the equator. This behavior is illustrated by comparing the $\Gamma_{N, Q=0}$ of Fig. 14 in all regions that have polar o points with their corresponding $\Gamma_{N, Q}$ for $Q>0$ depicted in Fig. 15. For regions with an
X-point separatrix and associated classical double degeneracy (Z.Lim, Z.R, $\perp$ Z.Int, $\perp$ S.Int, and S.R), there exists a certain $|Q|=Q_{\text {crit }}$ for which a migrating o point reaches the same location as the $\mathrm{x}$ point. When this occurs, the fixed points "annihilate," removing both the separatrix and the associated double degeneracy for all $|Q|>Q_{\text {crit. }}$. For perpendicular fields,

$$
Q_{\text {crit }}=\frac{N|\beta \pm \alpha|}{\sqrt{\beta^{2}-\alpha^{2}}} .
$$

The appropriate sign choice in Eq. (74) is determined by the requirement $|Q| \leq N$ to be - for $\theta<\theta_{1}$ and + for $\theta>\theta_{2}$. As the fields are tilted from perpendicular, the value of $Q_{\text {crit }}$ decreases, and there exist values of $\chi$ for which $Q_{\text {crit }}=0$ where the degenerate regions collapse to a pinched level set. This occurs on the boundaries between Z.R and I.Z and S.R and I.S (online black and blue curves in Fig. 13). At these boundaries, the doubly degenerate inner triangular subareas of spectra collapse to a single value of $\left(Q, h_{2}\right)$ marked with a (cyan online) diamond.

In all regions, except the Stark limit, when $Q_{\text {crit }}<Q<N$, $\Gamma_{N, Q}$ has two nonpolar o points, located at the maximum and minimum values of $h_{2}$. Although they are not explicitly depicted, these contours are similar to the contours depicted in Fig. 15, I.Z, II, I.S and $\perp$ M.Int. At these large values of $Q$, only the locations of the o points differ from region to region. For perpendicular fields both o points lie on the equator, and for large $\chi$ they remain very near to the poles.

As an example, consider how a spectrum in the Stark region S.R varies with $Q$. A sample spectrum is plotted over 
the region labeled S.R on the map (Fig. 13) or one may be viewed in Fig. 11 of Ref. [14]. We see that the small classically doubly degenerate triangular region exists only for a certain range of $Q$, and at $Q=0$ the degeneracy occupies its maximal energy range. $Q_{\text {crit }}$ is the value of $|Q|$ where the upper boundary, marking the energy of the maximal o point, and lower boundary, marking the energy of the x-point separatrix, meet. As was explained, this is the value of $Q$ where the o point and the $\mathrm{x}$ point annihilate, removing the degeneracy for $|Q|>Q_{\text {crit. }}$. The corresponding pictures on $\Gamma_{N, Q}$ are found in Fig. 14 (S.R) for $Q=0$ and Fig. 15 (S.R) for $|Q|$ $<Q_{\text {crit }}$, and for $|Q|>Q_{\text {crit }}, \Gamma_{N, Q}$ would look most similar to Fig. 15 (I.S).

\section{Map reconsidered}

Having considered all the different topological structures of the fully reduced Hamiltonian found in near-perpendicular electric and magnetic fields, we can now look back at the curves dividing the regions of the map and better understand how they are related to topological changes in $h_{2}$. (i) On $\chi^{Z}$ for $\theta<\theta_{1}$, where in Fig. 13 Z.R changes to I.Z, the X-point separatrix on $\Gamma_{N, Q}$ at $Q=0$ (Fig. 14, Z.R) collides with the southern polar o point, creating one singly pinched torus (Fig. 14, I.Z). In this process, the rotators near the south pole, which had overlapped in energy with those near the north pole, disappear. As a result the triangular classically doubly degenerate region of the spectrum shown in Fig. 13, Z.R collapses into a single monodromy point (cyan diamond in Fig. 13, I.Z). (ii) On $\chi^{S}$ for $\theta>\theta_{2}$, when we go from S.R to I.S, a similar process occurs on $\Gamma_{N, Q}$ at $Q=0$ near the north pole. (iii) On $\chi^{Z}$ for $\theta>\theta_{1}$, where in Fig. 13 I.Z changes to II and $\mathrm{R}$ changes to I.S, the minimum energy polar o point on $\Gamma_{N, Q}$ at $Q=0$ bifurcates into a nonpolar minimum o point and a singly pinched torus. This is depicted in Fig. 14 where the minimum energy o point at the north pole in I.Z bifurcates as we pass into region II into the singly pinched torus at the north pole, and a minimum o point which has migrated away from the pole. (iv) On $\chi^{S}$ for $\theta<\theta_{2}$, where I.S changes to II and $\mathrm{R}$ changes to I.Z, a similar process happens to the maximum o point on $\Gamma_{N, Q}$ at $Q=0$. This is depicted in Fig. 14 where the maximum energy o point at the south pole in I.S bifurcates in II into the singly pinched torus at the south pole, and a maximum o point which has migrated away from the pole. These considerations give a complete explanation of the spectra that were displayed in Ref. [14].

\section{QUANTUM CORRELLATION DIAGRAMS}

As the field parameters are varied across boundaries of the map (Fig. 13) the topological structure of the contours of $h_{2}\left(W, \delta_{W}\right)$ can change abruptly. However, the quantum energy eigenvalues are always continuous and even differentiable functions of $\chi$ and $\theta$. In this section we examine how the eigenvalues evolve from region to region on the map.

\section{A. Degeneracy breaking: From perpendicular to near- perpendicular fields}

Near the Zeeman and Stark limits, the quantum energy spectra have a structure in the second-order splitting associ-
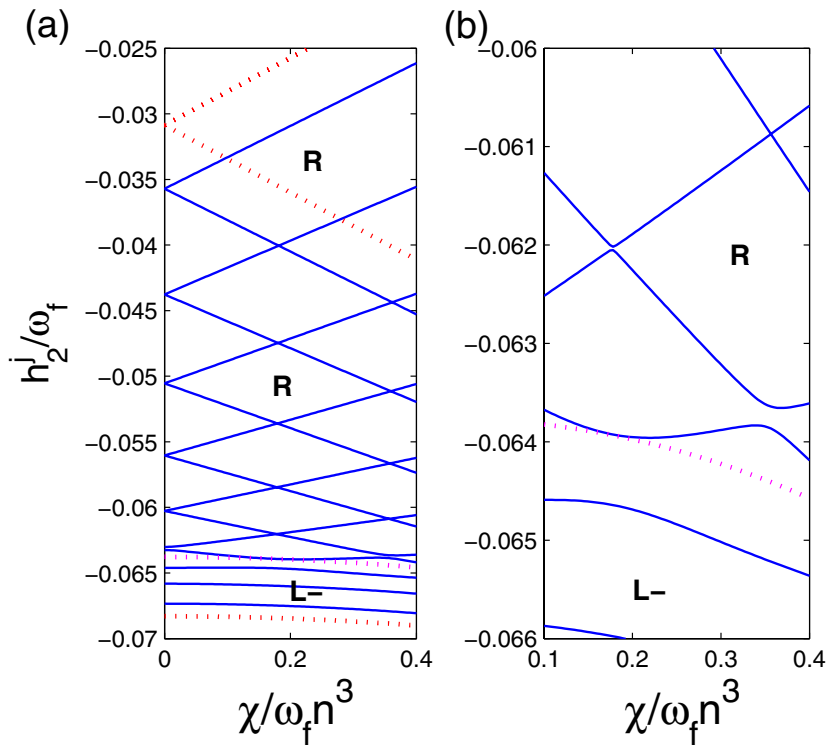

FIG. 16. (Color online) Correlation diagram from perpendicular to near-perpendicular fields. The energy eigenvalues of the 15 eigenstates at $n=15, q=0$ for $\theta=80^{\circ}, \omega_{f} n^{3}=0.01$ are plotted as functions of $\chi / \omega_{f} n^{3}$ using 111 evenly spaced data points. The quantum basis included $n$-manifolds $12-18$. Eigenstates that are (very nearly) degenerate at $\chi=0$ are split and anticross as the fields are tilted from perpendicular. (a) The four dashed lines are the classical energies of (from top to bottom): an effective o point at the south pole, an effective o point at the north pole, an x-point separatrix (magenta online), and a nonpolar o point having the minimum energy on $\Gamma_{N, Q}=\Gamma_{15,0}$. All energies between those of the north pole and of the $\mathrm{x}$-point separatrix are classically doubly degenerate. (b) Detail near the energy of the $\mathrm{x}$ separatrix reveals that anticrossings between the states that occur well inside the classically degenerate region are very narrow, but become larger near the $\mathrm{x}$-point separatrix.

ated with a double degeneracy. For perpendicular fields, the degeneracy in these regions is (very nearly) exact, and when the fields are tilted into nearly perpendicular configurations, the degeneracy is broken. As $\chi$ is varied, the formerly degenerate pair are displaced to higher and lower energies. If multiple adjacent degenerate pairs exist for $\chi=0$, then as $\chi$ is varied such states can exhibit anticrossings [41]. An example of such behavior appears in Fig. 16, where the solid curves depict the energies of 15 eigenstates from the $q=0$ manifold near the Stark limit as $\chi$ is increased from zero $(\perp$ S.Int $\rightarrow$ S.R). The energies of the 12 states associated with the anticrossings have a markedly different behavior from the three lowest-energy states which are nondegenerate at $\chi=0$. The detail in Fig. 16(b) illustrates that anticrossings between members of the 12 high-energy states are very narrow for $h_{2} / \omega_{f}>-0.063$ and widen near the three low-energy states as is found near $h_{2} / \omega_{f}=-0.0637, \chi / \omega_{f} n^{3}=0.35$. Near $h_{2} / \omega_{f}=-0.064, \chi^{\prime} \omega_{f} h^{3}=0.17$ the lowest-energy state above the (magenta online) dashed line behaves as if reflected from the three nondegenerate states below.

The nature of the states in such degenerate portions of the spectrum can be understood in terms of the classical degeneracy found on $\Gamma_{N, Q}$. In a primitive semiclassical approximation, a quantum state is associated with each contour of $h_{2}$ 
with $J_{+}$or $J_{-}$quantized as in Eq. (57c). For perpendicular fields $h_{2}$ [Eq. (35)] is symmetric about the equator $W=0$. Then, for some $\mathbf{F}$ and $\mathbf{B}$ there are two disjoint classical contours, $c^{(N)}$ localized in the north $(W>0)$ and $c^{(S)}$ localized in the south $(W<0)$, which have the same value of $h_{2}$. For every northern contour $c^{(N)}$ having an appropriately quantized primitive action, there is a corresponding southern contour $c^{(S)}$ with the same action $J_{ \pm}^{(N)}=J_{ \pm}^{(S)}$, forming a degenerate pair of states. When the fields are tilted from perpendicular, the $W$ symmetry is broken but there may still be regions of classical double degeneracy. However, in this case if a contour in the north has a particular value of $J_{ \pm}$, the contour in the south having the same energy does not necessarily have the same value of $J_{ \pm}$. Thus in general the degeneracy in the quantum states is removed as $\chi$ is varied. Continuing to tilt the fields may produce nonzero values of $\chi$ for which the difference in the primitive actions $J_{ \pm}^{(N)}-J_{ \pm}^{(S)}$ is an integer. In this case both contours are associated with a quantum state having the same energy, producing again a double degeneracy. Since the primitive semiclassical approximation does not account for tunneling, northern states are decoupled from southern states, and the locations where the semiclassical levels cross indicates the locations of the quantum anticrossings.

Looking back to Fig. 16, we identify the four dashed lines from top to bottom with the energies of the structures in Fig. $14 \perp$ S.Int and S.R: the o point at the south pole, the o point at the north pole, the x-point separatrix (magenta online), and the nonpolar o-point minimum. States with energies below that of the separatrix correspond to apparent librators while states above correspond to apparent rotators. From the semiclassical model [or simply from correspondence with $\partial h / \partial \chi=-2 \sin (2 \theta) W$ when not near an anticrossing], we identify the northern (southern) rotators with states having energies that are decreasing (increasing) functions of $\chi$. The anticrossings in the classically degenerate region which widen near the $\mathrm{x}$ separatrix are expected from tunneling between the northern and southern rotators, which would be strongest near the separatrix. As was discussed in Sec. VIII $\mathrm{B}$, as $\chi$ increases the energy range and phase-space area of the northern rotators are diminished, but the energy range and phase-space area of the southern rotators are increased. The semiclassical approximation predicts that northern rotators should disappear while new southern rotators should appear near the energy of the $\mathrm{x}$ separatrix. The analogous quantum process is present in Fig. 16(b) near $h_{2} / \omega_{f}=-0.0642$, $\chi / \omega_{f} n^{3}=0.19$, where the lowest-energy northern rotator changes character from a decreasing function of $\chi$ to an increasing function of $\chi$ near the energy of the separatrix.

Consider quantum expectation values of the operator $\omega_{f} \hat{W}$ [Eq. (62)] corresponding to the classical coordinate $W$. The scaled expectation value is

$$
w_{j}=\frac{1}{\omega_{f}(\bar{n})}\left\langle\Psi_{j}\left|\omega_{f} \hat{W}\right| \Psi_{j}\right\rangle .
$$

For perpendicular fields, the calculated degenerate quantum states are linear combinations of the northern and southern states corresponding to the odd and even parity about $W=0$, and the expectation value [Eq. (75)] vanishes. In between each anticrossing, the expectation [Eq. (75)] displays the northern or southern character of each quantum state. The quantum states are strongly coupled again at each anticrossing, where states adjacent in energy exchange their northern and southern character. The expectations of $w_{j}$ [Eq. (75)] for both states in an anticrossing are exchanged within an interval of $\chi / \omega_{f} n^{3}$ that is inversely proportional to the energy width of the anticrossing. These phenomena are illustrated in Fig. 17(a) where $w_{j}$ is plotted for the same states that appear in the energy correlation diagram in Fig. 16.

To visualize the $W$ character of quantum states in a spectrum, one may augment the quantum lattice such that a dot for each quantum state $\left|\Psi_{j}\right\rangle$ is plotted at the values $\left(q_{j}, h_{2}^{j}, w_{j}\right)$. In Fig. 17(b) we plot an augmented lattice for a spectrum with a classical double degeneracy but no quantum degeneracy, and away from any anticrossing. The lattice points are spaced regularly, and in the limit $n \rightarrow \infty$ at fixed $\omega_{f} n^{3}$ can be considered to define a surface in three dimensions. The projection of the augmented lattice into the $\left(Q, h_{2}\right)$ plane recovers the usual quantum lattices, but suggests that two families of quantum states exist within the triangular classically degenerate regions, and that each family requires a separate lattice coordinate system. Furthermore, one such coordinate system is connected continuously with the states in the rest of the spectrum, while the other is confined within the triangular region. This situation is precisely what results when constructing such a coordinate system from the classical actions.

\section{B. From the Zeeman limit to the Stark limit in near- perpendicular fields}

We now consider the correlation between the energy eigenstates in the Zeeman limit and the energy eigenstates in the Stark limit as we continuously vary the field parameters from $\mathbf{F}=0$ to $\mathbf{B}=0$ at a constant value of $\chi$ in the nearperpendicular region.

In Fig. 18 we trace the evolution of the 15 eigenstates associated with the $n=15$ and $q=0$ manifolds as $\theta$ is varied from $0^{\circ}$ to $90^{\circ}$, holding both $\omega_{f}=0.01 / n^{3}$ and $\chi=0.2 \omega_{f} n^{3}$ fixed. Each of the 15 states corresponds to a contour on $\Gamma_{N, Q}$ at $Q=0$ (Fig. 14) as the structure of the contours changes continuously from Z.Lim to S.Lim. As we move from left to right in Fig. 18, the corresponding series of diagrams in Fig. 14 are from Z.Lim to Z.R, across the upper row of pictures to S.R and then to S.Lim. Energies of classical level sets which have at least one component that is not a 2-torus are marked with dashed lines or diamonds.

At the Zeeman limit $\theta=0^{\circ}$ (Z.Lim), the four states with least energy are associated with pairs of degenerate apparent rotators, separated from 11 apparent librators at high energies by an x-point separatrix, with energy marked by a dashed line (magenta online).

When $\theta$ is increased holding $\chi / \omega_{f} n^{3}=0.2$ fixed, the degeneracy in the rotators is broken (Z.R). The northern rotators move to lower energies while the southern rotators move to higher energies, resulting in avoided crossings which are narrow except when they occur at energies close to the sepa- 


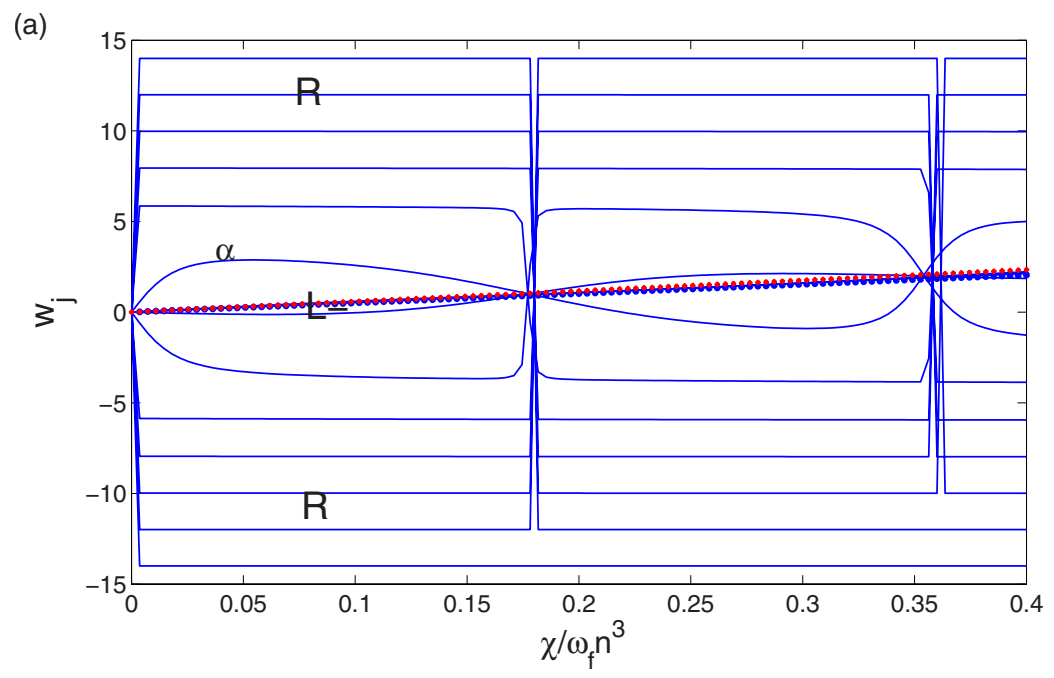

(b)

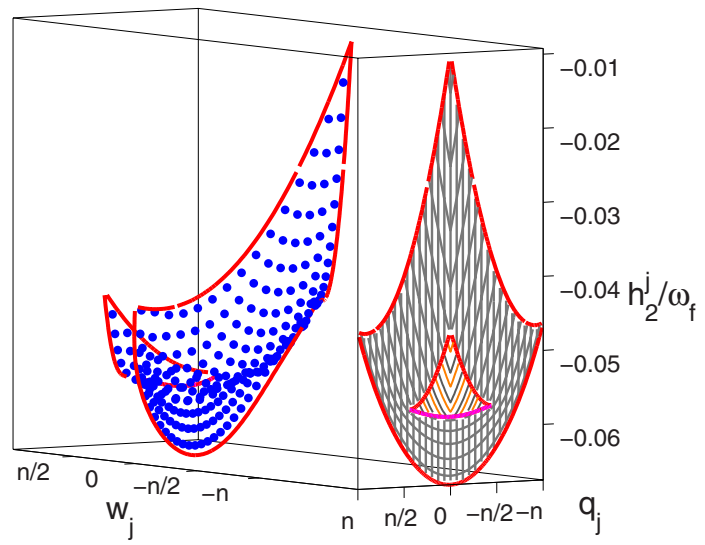

FIG. 17. (Color online) (a) The expectation values $w_{j}=\left\langle\psi_{j}\left|\omega_{f} \hat{W}\right| \psi_{j}\right\rangle / \omega_{f}$ for the 15 energy eigenstates at $n=15, q=0$ for $\theta=80^{\circ}, \omega_{f} n^{3}$ $=0.01$ are plotted as functions of $\chi^{/} \omega_{f} n^{3}$ with 111 evenly spaced data points (compare with Fig. 16). The quantum basis included $n$-manifolds $12-18$. (a) At $\chi=0$ doubly degenerate eigenstates are coupled by tunneling into symmetric and antisymmetric combinations of northern and southern states such that $w_{j}=0$. As the fields are tilted from perpendicular, the $w_{j}$ expectations assume values that are in agreement with the localization of the semiclassical states on $\Gamma_{N, Q}$. For $\chi / \omega_{f} N^{3}<0.1$ there are expectation values associated with six northern and six southern rotators, and three librators which very closely follow the classical expectations of $W$ for the minimum o point plotted in (blue online) dots and the x point plotted with (red online) crosses. At energy anticrossings, the quantum states (adiabatically) exchange their $W$ character, with narrow anticrossings between rotators in the far north and far south occurring within small intervals of $\chi / \omega_{f} N^{3}$, and wider anticrossings occurring between rotators near the $W$ locations of the x-point separatrix. The state labeled $\alpha$ is a northern rotator which changes character to a southern rotator at $\chi / \omega_{f} N^{3} \approx 0.17$ where it crosses the $W$ of the x point, mildly affecting the librator, which visibly oscillates about the x point. The very slow $W$ exchange between the southernmost northern rotator, and the northernmost southern rotator at $\chi / \omega_{f} N^{3} \approx 0.36$ is identified with the wide energy anticrossing in Fig. 16(b) at $\left(h_{2} / \omega_{f}, \chi / \omega_{f} N^{3}\right) \approx(-0.0637,0.35)$. (b) An augmented quantum lattice is constructed by plotting a dot for each quantum eigenstate $\left|\psi_{j}\right\rangle$ at the coordinates $\left(q_{j}, h_{2}^{j}, w_{j}\right)$. Classical boundaries are plotted at their classical expectation values of $W$. The augmented lattice aids in clarifying the nature of the triangular classically degenerate regions which occur in the two-dimensional quantum lattices, and suggests that such quantum lattice vector transport can be well defined when they pass through the boundaries of the triangle that are not associated with the classical x-point separatrix.

ratrix. The lowest two dashed red lines are the energies of the polar effective o points. The energy of the southern pole climbs to higher energies and meets the descending $\mathrm{x}$ separatrix at $\theta \approx 29^{\circ}$, removing the classical double degeneracy. As we would expect from the semiclassical model, the northern rotators pass uneventfully through the energy of the south pole, while southern rotators experience interactions as they cross the $\mathrm{x}$ separatrix to become librators.

Upon passing $\theta \approx 29^{\circ}$, the $\mathrm{x}$-point separatrix and the south polar o point collapse into a singly pinched torus, the energy of which is marked with a string of (cyan online) diamonds
(I.Z). Distinction between the energy evolution of apparent librators above the diamonds and the apparent rotators below the diamonds is no longer present. This is expected because this region is devoid of degeneracy, and we do not expect groups of states here to have vastly different sharp $W$ localizations.

At $\theta \approx 39^{\circ}$, the o point at the north pole splits into a nonpolar o point and a singly pinched torus (II). States that have energies between the energies of the pinched tori are apparent rotators, while those above and below are apparent librators. However there is no real distinction between these, and 
(a)

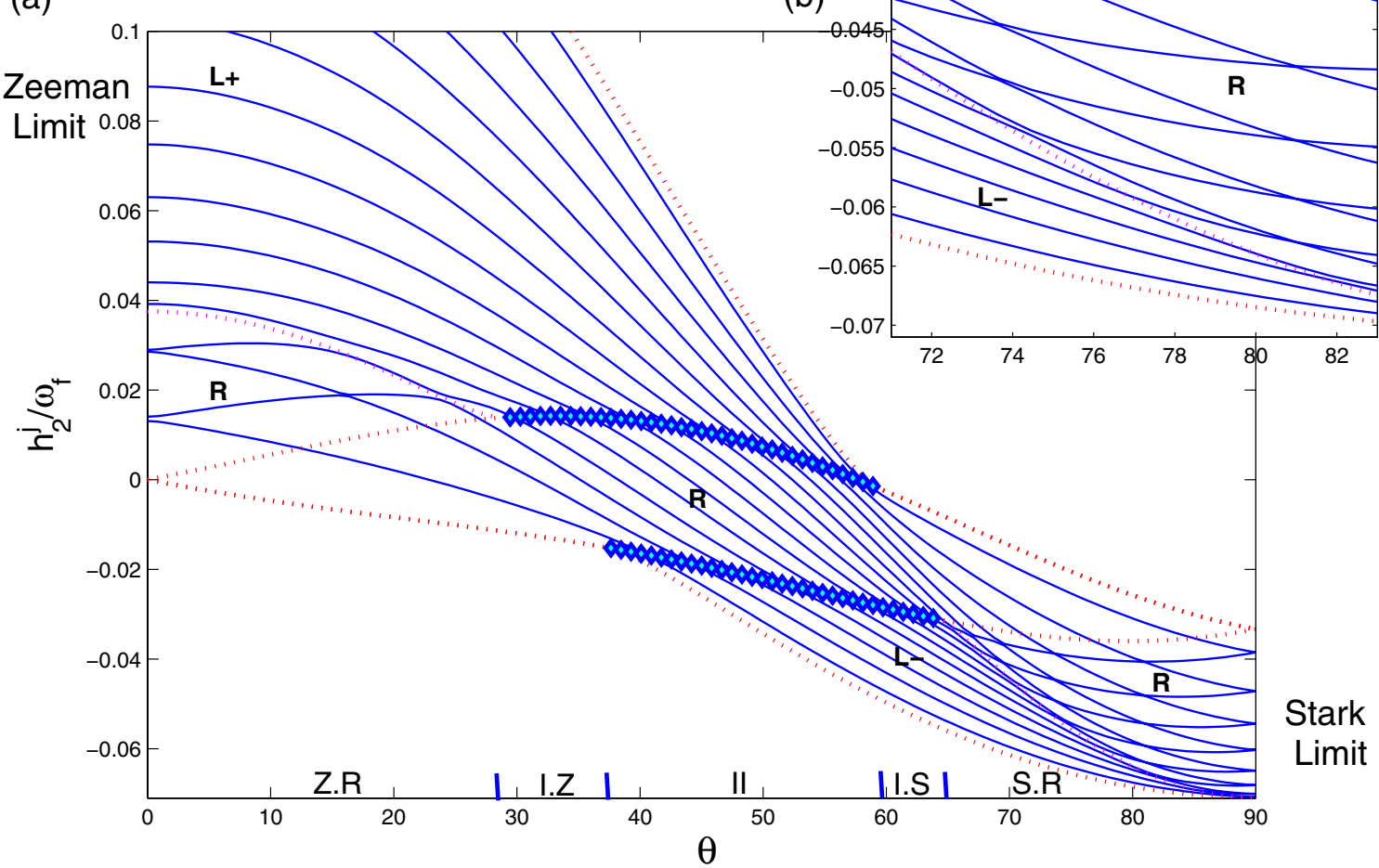

FIG. 18. (Color online) Correlation diagram from the Zeeman to the Stark limit in near-perpendicular fields. The energy eigenvalues of the 15 eigenstates at $n=15, q=0, \omega_{f} n^{3}=0.01$ are plotted as functions of $\theta$ in near-perpendicular fields $\chi / \omega_{f} n^{3}=0.2$. The quantum basis included $n$-manifolds $12-18$. The evolution of the energies of the states is traced with solid (blue online) lines from the Zeeman limit (Z.Lim) $\theta=0^{\circ}$ to $\theta=90^{\circ}$ at the Stark limit (S.Lim), passing into the regions (Z.R, I.Z, II, I.S, and S.R) at approximately $\theta$ $=\left(0^{\circ}, 29^{\circ}, 38^{\circ}, 59^{\circ}, 64^{\circ}\right.$, and $\left.90^{\circ}\right)$ en route. Energies for which at least one component of the classical level set is not a 2-torus are marked with dashed curves or curves of diamonds. The upper (lower) curve of (cyan online) diamonds represents the energy of the singly pinched torus at the south (north) pole, and joins smoothly to (red online) dashed curves which mark the energy of a polar effective o point. The (magenta online) dashed curves joining the curve of diamonds mark the energy of an x-point separatrix, and all energies between an x-point energy and the nearest effective o-point energy are classically doubly degenerate. For $\theta \leq 59^{\circ}\left(\theta \geq 29^{\circ}\right)$, the dashed curve with maximum (minimum) energy is that of the Zeeman (Stark) librator o point. Regions of states separated by the energies of classical structures are marked with either an $R$, an $L_{+}$, or an $L_{-}$, indicating that they are associated with apparent rotators, apparent librators about a maximal energy, and apparent librators about a minimal energy, respectively. In the upper right-hand corner of the figure, detail near the Stark limit is enlarged. States associated with apparent rotators in classically doubly degenerate regions exhibit narrow anticrossings, which widen near the energy of the x-point separatrix. As the energy range of degeneracy in the Zeeman (Stark) region is diminished with increasing (decreasing) $\theta$, states associated with southern (northern) rotators pass through the energy of the $\mathrm{x}$ separatrix and change character to librators, while northern (southern) rotators continue unaffected into nondegenerate regions below (above) the energy of the effective o point at the south (north) pole.

in Fig. 18 the quantum energies pass with little disturbance through the energy of a pinched torus as they change apparent character between rotators and librators. This passage is in contrast with changes of character which occur as energies cross an $\mathrm{x}$-point separatrix.

At $\theta \approx 59^{\circ}$, the maximal energy o point supporting the Zeeman librators combines with the pinched torus at the south pole into an o point with maximal energy (I.S). The singly pinched torus at the north pole divides the southern rotators from the low-energy Stark librators.

At $\theta \approx 64^{\circ}$ the pinched torus at the north pole splits into an $x$ point and an effective polar o point (S.R). As $\theta$ continues to increase, the north polar o point climbs in energy to meet that of the south pole, while the energy of the $\mathrm{x}$ point decreases to join that of the minimal o point. The $\mathrm{x}$ point separates the low-energy Stark librators from the high-energy Stark rotators. At all energies between that of the $\mathrm{x}$ point and the north pole, there is a classical double degeneracy, and the associated narrow anticrossing behavior.

When the Stark limit (S.Lim) is reached at $\theta=90^{\circ}$, every state except that with the lowest energy is paired in a double degeneracy associated with a northern-southern rotator pair.

\section{CONCLUSION}

We have used classical perturbation theory to derive an integrable system which describes the dynamics of the hydrogen atom in weak near-perpendicular electric and magnetic fields. Semiclassical quantization of the resulting integrable system is in good agreement with quantum calculations, and provides interpretation of the structures in the second-order quantum spectrum that were predicted but not explained in Ref. [14]. 


\section{ACKNOWLEDGMENTS}

The authors wish to thank Nahum Zobin, David Lutzer, Sarah Day, and George Rublein for several helpful discussions. This work was supported by the National Science Foundation.

\section{APPENDIX A: KEPLER AVERAGING}

\section{Average in the orbital frame}

To average functions of phase space over an unperturbed Kepler orbit; use the identity $M=N e$ and the eccentric anomaly $\Psi$, defined by Kepler's equation $\phi_{N}=\Psi-\frac{M}{N} \sin \Psi$, to rewrite Eq. (6d) as [43]

$$
\langle g\rangle_{\phi_{N}}=\frac{1}{2 \pi} \int_{0}^{2 \pi} g\left(1-\frac{M}{N} \cos \Psi\right) d \Psi .
$$

The functions to be averaged depend on the position vector and tensor components $\left(x_{j}\right.$ and $\left.x_{i} x_{j}\right)$ in the space-fixed frame with basis vectors $\{\hat{\mathbf{x}}, \hat{\mathbf{y}}, \hat{\mathbf{z}}\}$. These components can be expressed in terms of the orbital reference frame components $\left(x_{j}^{\prime}\right.$ and $\left.x_{i}^{\prime} x_{j}^{\prime}\right)$ with basis vectors $\left\{\hat{\mathbf{x}}^{\prime}, \hat{\mathbf{y}}^{\prime}, \hat{\mathbf{z}}^{\prime}\right\}=\left\{\frac{\mathbf{M}}{M}, \frac{\mathbf{L} \times \mathbf{M}}{L M}, \frac{\mathbf{L}}{L}\right\}$ using the orthonormal direction cosine matrix transformation

$$
R_{i, j}=\hat{\mathbf{x}}_{i} \cdot \hat{\mathbf{x}}_{j}^{\prime} .
$$

Since the orbit is fixed, the Kepler averages Eq. (A1) of $x_{j}$ and $x_{i} x_{j}$ reduce to averages in the orbital frame,

$$
\begin{gathered}
\left\langle x_{j}\right\rangle_{\phi_{N}}=R_{j, k}^{T}\left\langle x_{k}^{\prime}\right\rangle_{\phi_{N}}, \\
\left\langle x_{i} x_{j}\right\rangle_{\phi_{N}}=R_{i, k}^{T}\left\langle x_{k}^{\prime} x_{p}^{\prime}\right\rangle_{\phi_{N}} R_{p, j} .
\end{gathered}
$$

The averages in the orbital frame may be computed by expressing the $x_{j}^{\prime}$ and $x_{i}^{\prime} x_{j}^{\prime}$ components in terms of the eccentric anomaly using [44]

$$
\mathbf{r}^{\prime}=\left(x^{\prime}, y^{\prime}, z^{\prime}\right)=\left[N^{2}\left(\cos \Psi-\frac{M}{N}\right), N L \sin \Psi, 0\right] \text {. }
$$

\section{Computing Eqs. (6) of Sec. II A}

To compute Eq. (6b), average $H_{1}$ as it appears in Eq. (2) using $\langle\mathbf{L}\rangle_{\phi_{N}}=\mathbf{L}$, and Eq. (A3a) with $R_{1,1}^{T}=\mathbf{M}_{x} / M$ and the average of Eq. (A4),

$$
\left\langle\mathbf{r}^{\prime}\right\rangle_{\phi_{N}}=\left(-\frac{3}{2} N M, 0,0\right) .
$$

The result is $\left\langle H_{1}\right\rangle_{\phi_{N}}$ in Eq. (12).

The average of $\mathrm{H}_{2}$ as it appears in Eq. (2) is

$$
\left\langle H_{2}\right\rangle_{\phi_{N}}=\frac{B^{2}}{8}\left(\langle x x\rangle_{\phi_{N}}+\langle y y\rangle_{\phi_{N}}\right)+F_{z}\langle z\rangle_{\phi_{N}},
$$

and is computed by noting that the only nonzero Kepleraveraged $x_{i}^{\prime} x_{j}^{\prime}$ are (using $M^{2}+L^{2}=N^{2}$ )

$$
\left\langle x^{\prime} x^{\prime}\right\rangle_{\phi_{N}}=\frac{N^{2}}{2}\left(N^{2}+4 M^{2}\right) \text {, }
$$

$$
\left\langle y^{\prime} y^{\prime}\right\rangle_{\phi_{N}}=\frac{N^{2}}{2} L^{2} .
$$

After expressing the averages in the orbital frame via Eqs. (A3) and (A7) one obtains:

$$
\begin{aligned}
\left\langle H_{2}\right\rangle_{\phi_{N}}= & \frac{B^{2}}{8}\left\langle x^{\prime} x^{\prime}\right\rangle_{\phi_{N}}\left(R_{1,1}^{T}{ }^{2}+R_{2,1}^{T}{ }^{2}\right)+\frac{B^{2}}{8}\left\langle y^{\prime} y^{\prime}\right\rangle_{\phi_{N}} \\
& \times\left(R_{1,2}^{T}{ }^{2}+R_{2,2}^{T}{ }^{2}\right)+F_{z} R_{3,1}^{T}\left\langle x^{\prime}\right\rangle_{\phi_{N}} .
\end{aligned}
$$

The $F_{z}$ term is $-\frac{3}{2} N F_{z} M_{z}$, while the term proportional to $B^{2}$ may be reexpressed by substitution using $\Sigma_{j}\left(R_{i, j}^{T}\right)^{2}$ $=\Sigma_{i}\left(R_{i, j}^{T}\right)^{2}=1$, which follows from the orthonormality of $R$. One obtains

$$
\begin{aligned}
\left\langle H_{2}\right\rangle_{\phi_{N}}= & -\frac{3}{2} N F_{z} M_{z}+\frac{B^{2}}{8}\left\langle x^{\prime} x^{\prime}\right\rangle_{\phi_{N}}+\frac{B^{2}}{8}\left[\left(\left\langle y^{\prime} y^{\prime}\right\rangle_{\phi_{N}}\right.\right. \\
& \left.\left.-\left\langle x^{\prime} x^{\prime}\right\rangle_{\phi_{N}}\right) R_{3,1}^{T}{ }^{2}+\left\langle y^{\prime} y^{\prime}\right\rangle_{\phi_{N}} R_{3,3}^{T}{ }^{2}\right] .
\end{aligned}
$$

After substitution of Eqs. (A7) and $R_{3,1}^{T}=\mathbf{M}_{z} / M$ and $R_{3,3}^{T}$ $=\mathbf{L}_{z} / L$, the result is $\left\langle H_{2}\right\rangle_{\phi_{N}}$ in Eq. (12).

\section{a. The explicit generating function $f_{1}$}

The terms in $H_{2}^{\prime}$ require the first-order generating function $f_{1}\left(f_{2}\right.$ is not needed because $\left\langle\partial f_{2} / \partial \phi_{N}\right\rangle_{\phi_{N}}=0$ by the imposed periodicity of $f$ ). To obtain an explicit functional form for $f_{1}$, we first note that Eqs. (5b) and (6b) together imply the following partial differential equation in the Delaunay coordinates:

$$
\frac{\partial f_{1}}{\partial \phi_{N}}=N^{3}\left(\left\langle H_{1}\right\rangle_{\phi_{N}}-H_{1}\right)=N^{3} F_{x}\left(\langle x\rangle_{\phi_{N}}-x\right) .
$$

The last equality is obtained from the difference of $\left\langle H_{1}\right\rangle_{\phi_{N}}$ in Eq. (12) and $H_{1}$ in Eq. (2) and is independent of $B$ because $\left\langle L_{z}\right\rangle_{\phi_{N}}=L_{z}$. We obtain an explicit form for $f_{1}$ by the indefinite integration of Eq. (A10) over $\phi_{N}$, holding the rest of the Delaunay variables fixed. Again utilizing the eccentric anomaly $\Psi$, one obtains

$$
\begin{aligned}
f_{1}= & -N^{5} F_{x} R_{1,1}^{T}\left[\left(1-\frac{M^{2}}{2 N^{2}}\right) \sin \Psi-\frac{M}{2 N} \sin \Psi \cos \Psi\right] \\
& -N^{4} L F_{x} R_{1,2}^{T}\left(1-\frac{M}{2 N}-\cos \Psi+\frac{M}{2 N} \cos ^{2} \Psi\right)+C,
\end{aligned}
$$

which is of the form

$$
f_{1}=\tilde{f}_{1}+C
$$

where the undetermined integration constant $C$ is constrained only by the requirement that it be independent of $\phi_{N}$. We are free to choose

$$
C=-\left\langle\tilde{f}_{1}\right\rangle_{\phi_{N}}=N^{4} L F_{x} R_{1,2}^{T}\left(1+\frac{M}{4 N}\right)
$$

such that

$$
\left\langle f_{1}\right\rangle_{\phi_{N}}=0
$$




\section{b. The second-order Hamiltonian is independent of $F_{x} B$}

Substituting Eq. (A12) and $H_{1}$ as it appears in Eq. (2) into Eq. (5d) produces an expression for $H_{2}^{\prime}$ that has an average of the form

$$
\begin{aligned}
\left\langle H_{2}^{\prime}\right\rangle_{\phi_{N}}= & F_{x}\left(\left\langle\frac{\partial x}{\partial N} \frac{\partial \tilde{f}_{1}}{\partial \phi_{N}}\right\rangle_{\phi_{N}}+\left\langle\frac{\partial x}{\partial L} \frac{\partial \tilde{f}_{1}}{\partial \phi_{p}}\right\rangle_{\phi_{N}}\right. \\
& \left.+\left\langle\frac{\partial x}{\partial L_{z}} \frac{\partial \tilde{f}_{1}}{\partial \Omega}\right\rangle_{\phi_{N}}\right)-F_{x}\left(\frac{\partial\langle x\rangle_{\phi_{N}}}{\partial L} \frac{\partial\left\langle\tilde{f}_{1}\right\rangle_{\phi_{N}}}{\partial \phi_{p}}\right. \\
& \left.+\frac{\partial\langle x\rangle_{\phi_{N}}}{\partial L_{z}} \frac{\left.\partial \tilde{f}_{1}\right\rangle_{\phi_{N}}}{\partial \Omega}\right)-\frac{3}{2 N^{4}}\left\langle\left(\frac{\partial \tilde{f}_{1}}{\partial \phi_{N}}\right)^{2}\right\rangle_{\phi_{N}}
\end{aligned}
$$

Since $\tilde{f}_{1}$ is proportional to $F_{x},\left\langle H_{2}^{\prime}\right\rangle_{\phi_{N}}$ is composed entirely of terms proportional to $F_{x}^{2}$. Thus, the second-order dynamics of hydrogen in near-perpendicular fields has been rendered independent of the mixed field term $F_{x} B$ by our choice of integration constant $C$ in Eq. (A13).

\section{c. Computing $\left\langle\boldsymbol{H}_{2}^{\prime}\right\rangle_{\phi_{N}}$}

The evaluation of Eq. (A15) is lengthy and tedious; we outline the essential steps here, and publish a detailed account as supplementary material [42].

After eliminating $M$ (via $\left.N^{2}=L^{2}+M^{2}\right), x$ and $\tilde{f}_{1}$ are functions only of $N$ and $L$ and the functions $R_{1,1}^{T}, R_{1,2}^{T}$, and $\Psi$. Their averages $\langle x\rangle$ and $\left\langle\tilde{f}_{1}\right\rangle$ are independent of $\Psi$ and are considerably reduced. The required partial derivatives of these four functions in the Delaunay coordinates are straightforward to compute; using implicit differentiation of Kepler's equation for calculating derivatives of $\Psi$, and maintaining the derivatives of the $R_{i, j}^{T}$ as formal functions. After substituting these partial derivatives into Eq. (A15) and performing the required averages, one obtains

$$
\begin{aligned}
\left\langle H_{2}^{\prime}\right\rangle_{\phi_{N}}= & \frac{F_{x}^{2} N^{4}}{16} L\left(5 N^{2}+3 L^{2}\right)\left(\frac{\partial R_{1,1}^{T}}{\partial L} \frac{\partial R_{1,2}^{T}}{\partial \phi_{p}}-\frac{\partial R_{1,2}^{T}}{\partial L} \frac{\partial R_{1,1}^{T}}{\partial \phi_{p}}\right. \\
& \left.+\frac{\partial R_{1,1}^{T}}{\partial L_{z}} \frac{\partial R_{1,2}^{T}}{\partial \Omega}-\frac{\partial R_{1,2}^{T}}{\partial L_{z}} \frac{\partial R_{1,1}^{T}}{\partial \Omega}\right) \\
& -\frac{F_{x}^{2} N^{4}}{16}\left(\left(5 N^{2}+4 L^{2}\right) R_{1,2}^{T}\right. \\
& \times \frac{\partial R_{1,1}^{T}}{\partial \phi_{p}}-5 L^{2} R_{1,1}^{T} \frac{\partial R_{1,2}^{T}}{\partial \phi_{p}}+\left(14 N^{2}+10 L^{2}\right) R_{1,1}^{T}{ }^{2} \\
& \left.+20 L^{2} R_{1,2}^{T}{ }^{2}\right) .
\end{aligned}
$$

The final simplifications come from the properties of $R^{T}$. Since $R^{T}$ is independent of $\phi_{N}$, the expression in large parentheses of the first term can be interpreted as the Poisson bracket, $\left\{R_{1,1}^{T}, R_{1,2}^{T}\right\}$ in the Delaunay variables. It is straightforward but tedious to verify the following identity [we find it is quickest to use the Euler matrix form of $R^{T}$ [45], elimi- nating the orbital inclination $i$ with the equation $i$ $\left.=\cos ^{-1}\left(L_{z} / L\right)\right]$ :

$$
\left\{R_{1,1}^{T}, R_{1,2}^{T}\right\}=-\frac{R_{1,3}^{T} 2}{L} .
$$

By the same suggested method, it is trivial to prove two more identities:

$$
\begin{gathered}
\frac{\partial R_{1,1}^{T}}{\partial \phi_{p}}=R_{1,2}^{T}, \\
\frac{\partial R_{1,2}^{T}}{\partial \phi_{p}}=-R_{1,1}^{T} .
\end{gathered}
$$

Substituting these identities into Eq. (A16), one obtains

$$
\begin{aligned}
\left\langle H_{2}^{\prime}\right\rangle_{\phi_{N}}= & -\frac{F_{x}^{2} N^{4}}{16}\left(5 N^{2}+3 L^{2}\right) R_{1,3}^{T}{ }^{2}-\frac{F_{x}^{2} N^{4}}{16}\left[\left(5 N^{2}+24 L^{2}\right) R_{1,2}^{T}{ }^{2}\right. \\
& \left.+\left(14 N^{2}+15 L^{2}\right) R_{1,1}^{T}{ }^{2}\right] .
\end{aligned}
$$

Eliminating $R_{1,2}^{T}{ }^{2}$ with the column orthonormality of $R^{T}$, and cosmetically eliminating $L^{2}$ in favor of $M^{2}$, one obtains

$$
\left\langle H_{2}^{\prime}\right\rangle_{\phi_{N}}=-\frac{F_{x}^{2} N^{4}}{16}\left(29 N^{2}-24 M^{2}-21 L^{2} R_{1,3}^{T}{ }^{2}+9 M^{2} R_{1,1}^{T}{ }^{2}\right) .
$$

Finally, with $R_{1,1}^{T}=M_{x} / M$ and $R_{1,3}^{T}=L_{x} / L$, we have arrived at $\left\langle H_{2}^{\prime}\right\rangle_{\phi_{N}}$ as it appears in Eq. (12).

\section{APPENDIX B: THE TOPOLOGY OF $\Gamma_{N, Q}$}

The space of all Kepler orbits at fixed $N$ is $\Gamma_{N} \sim S^{2} \times S^{2}$. Each point $K \in \Gamma_{N}$ is an equivalence class of points in the full six-dimensional Cartesian phase space such that all points $\left(x, y, z, p_{x}, p_{y}, p_{z}\right)$ contained in a particular Kepler orbit having the vectors $\mathbf{J}_{1}$ and $\mathbf{J}_{2}$ are equivalent [46]. Define a natural distance between two Kepler orbits:

$$
d_{K}(K, \tilde{K})=\left|\mathbf{J}_{1}-\widetilde{\mathbf{J}}_{1}\right|+\left|\mathbf{J}_{2}-\widetilde{\mathbf{J}}_{2}\right| .
$$

A Pauli orbit $P \in \Gamma_{N}$ is a subset of the space of all Kepler orbits at a fixed $N$ defined by

$$
\begin{aligned}
P \equiv & \left\{K \in \Gamma_{N} \mid Q=\mu_{1}+\mu_{2}, W=\mu_{1}-\mu_{2}, \text { and if }|W|<N\right. \\
& \left.-|Q| \text { then } \delta_{W}=\frac{1}{2}\left(\psi_{1}-\psi_{2}\right)\right\} .
\end{aligned}
$$

In $\Gamma_{N}$, each $P$ is either a one-dimensional closed curve or a point [if $Q= \pm N$ or $Q=0$ and $W= \pm(N-|Q|)$ ]. If $P$ is not a point, then each $K \in P \subset \Gamma_{N}$ is labeled by a value of the coordinate $\sigma_{Q}$.

Let the space of all Pauli orbits at a fixed $Q$ be denoted $\Gamma_{N, Q}$. Each point $P \in \Gamma_{N, Q}$ is an equivalence class of points $K \in \Gamma_{N}$ such that all points $K$ which are contained in the same $P$ defined in Eq. (B2) are equivalent. This structure, motivated by the perturbative description of the motion, determines the effective topology on the $\left(W, \delta_{W}\right)$ surface.

Proposition. $\Gamma_{N, Q}$, the two-dimensional fully reduced phase space at fixed $N$ and $Q$ [the $\left(W, \delta_{W}\right)$ surface] is homeomorphic to a sphere. 
The homeomorphism is given in spherical polar coordinates $(r, \theta, \phi)$ by

$$
\begin{gathered}
N-|Q|=r, \\
W=r \cos \theta, \\
\delta_{W}=\frac{\phi}{2} .
\end{gathered}
$$

To establish that neighborhoods of $\left(W, \delta_{W}\right)$ near $W= \pm(N$ $-|Q|)$ are connected like a sphere, we show that, given any $P$ and $\widetilde{P}$ such that (i) $Q=\widetilde{Q}$ with $|Q| \leq N$, (ii) $W=\widetilde{W}$ with $|W|$ $<N-|Q|$, but (iii) $\delta_{W} \neq \widetilde{\delta}_{W}$, then $\forall K \in P \exists \tilde{K} \in \widetilde{P}$ such that

$$
\lim _{W \rightarrow \pm(N-|Q|)} d_{K}(K, \tilde{K})=0 .
$$

To show this, obtain expressions for $J_{1, x_{i}^{\prime}}\left(Q, W, \delta_{Q}, \delta_{W}\right)$ and $J_{2, x_{i}^{\prime}}\left(Q, W, \delta_{Q}, \delta_{W}\right)$ from Eqs. (29) and (26), then substitute them into the definition of $d_{K}$ in Eq. (B1) and evaluate for the case $Q=\tilde{Q}, W=\tilde{W}$ to obtain

$$
\left.d_{K}\right|_{(Q, W)=(\tilde{Q}, \tilde{W})}=\sqrt{N^{2}-(Q+W)^{2}} \Theta_{1}+\sqrt{N^{2}-(Q-W)^{2}} \Theta_{2},
$$

where

$$
\Theta_{1}=\sqrt{\frac{1-\cos \left(\delta_{Q}+\delta_{W}-\tilde{\delta}_{Q}-\tilde{\delta}_{W}\right)}{2}}
$$

$$
\Theta_{2}=\sqrt{\frac{1-\cos \left(\delta_{Q}-\delta_{W}-\widetilde{\delta}_{Q}+\tilde{\delta}_{W}\right)}{2}} .
$$

For a given $\delta_{Q}$, Eq. (B5b) will vanish if

$$
\widetilde{\delta}_{Q}=\delta_{Q}+\delta_{W}-\widetilde{\delta}_{W}+2 \pi k_{1}
$$

and Eq. (B5c) will vanish if

$$
\widetilde{\delta}_{Q}=\delta_{Q}-\delta_{W}+\widetilde{\delta}_{W}+2 \pi k_{2}
$$

where $k_{i}=0, \pm 1, \pm 2, \ldots$. These two equations can be simultaneously satisfied only if $\left(\delta_{W}-\widetilde{\delta}_{W}\right)=k_{3} \pi \quad\left(k_{3}\right.$ $=0, \pm 1, \pm 2, \ldots)$. But since the $\delta_{W}$ coordinate is $\pi$ periodic, all such points mean that $\delta_{W}=\widetilde{\delta}_{W}$, and we recover the trivial fact that Pauli orbits with the same values of $\left(Q, W, \delta_{W}\right)$ are identified. But suppose $\delta_{W} \neq \widetilde{\delta}_{W}$ and consider the limit of Eq. (B5a) as $W$ approaches $\pm(N-|Q|)$ :

$$
\begin{aligned}
\lim _{W \rightarrow \pm(N-|Q|)} d_{K}= & 2(N \pm Q)(|Q| \mp Q) \Theta_{1} \\
& +2(N \mp Q)(|Q| \pm Q) \Theta_{2} .
\end{aligned}
$$

For $Q=0$ or $Q= \pm N$, both terms in Eq. (B7) vanish, consistent with the fact that at these four points the phase space consists of a single Kepler orbit. For all $Q$ with $0<|Q|<N$, either the term proportional to $\Theta_{1}$ or the term proportional to $\Theta_{2}$ will vanish as $W \rightarrow \pm(N-|Q|)$. The surviving term can always be made to vanish by taking the limit at the fixed value of $\widetilde{\delta}_{Q}$ such that the required equation (B6b) or (B6a) is satisfied. That is, for any $\delta_{Q}, \delta_{W}$, and $\widetilde{\delta}_{W}$, there exists a $\widetilde{\delta}_{Q}$, depending linearly on $\delta_{Q}$, such that the limit Eq. (B7) vanishes.
[1] Yu. N. Demkov, B. S. Monozon, and V. N. Ostrovskii, Sov. Phys. JETP 30, 775 (1970); T. P. Grozdanov and E. A. Solov'ev, J. Phys. B 15, 1195 (1982); J. B. Delos, S. K. Knudson, and D. W. Noid, Phys. Rev. A 28, 7 (1983); P. A. Braun and E. A. Solov'ev, Sov. Phys. JETP 59, 38 (1984); P. Schmelcher and L. S. Cederbaum, Chem. Phys. Lett. 208, 548 (1993); Phys. Rev. A 47, 2634 (1993); E. Flöthmann, J. Main, and K. H. Welge, J. Phys. B 27, 2821 (1994); J. Main and G. Wunner, ibid. 27, 2835 (1994); R. C. Hilborn, L. R. Hunter, K. Johnson, S. K. Peck, A. Spencer, and J. Watson, Phys. Rev. A 50, 2467 (1994); R. C. Hilborn, Am. J. Phys. 63, 330 (1995); J. von Milczewski, G. H. F. Diercksen, and T. Uzer, Phys. Rev. Lett. 76, 2890 (1996); J. Main, M. Schwacke, and G. Wunner, Phys. Rev. A 57, 1149 (1998); C. Jaffé, D. Farrelly, and T. Uzer, ibid. 60, 3833 (1999); J. Rao, D. Delande, and K. T. Taylor, J. Phys. B 34, L391 (2001); D. M. Wang and J. B. Delos, Phys. Rev. A 63, 043409 (2001); T. Bartsch, J. Main, and G. Wunner, ibid. 67, 063411 (2003); S. Gekle, J. Main, T. Bartsch, and T. Uzer, ibid. 75, 023406 (2007); and references that follow.

[2] W. Pauli, Z. Phys. 36, 336 (1926); B. L. Van der Waerden, Sources of Quantum Mechanics (Dover, New York, 1967) (English translation).
[3] F. Penent, D. Delande, F. Biraben, and J. C. Gay, Opt. Commun. 49, 184 (1984); F. Penent, D. Delande, and J. C. Gay, Phys. Rev. A 37, 4707 (1988); G. Wiebusch, J. Main, K. Krüger, H. Rottke, A. Holle, and K. H. Welge, Phys. Rev. Lett. 62, 2821 (1989); G. Raithel, M. Fauth, and H. Walther, Phys. Rev. A 44, 1898 (1991); G. Raithel and H. Walther, ibid. 49, 1646 (1994).

[4] D. A. Sadovskií and B. I. Zhilinskií, Phys. Rev. A 57, 2867 (1998); N. Berglund and T. Uzer, Found. Phys. 31, 283 (2001).

[5] P. Bellomo, C. R. Stroud, D. Farrelly, and T. Uzer, Phys. Rev. A 58, 3896 (1998).

[6] M. J. Gourlay, T. Uzer, and D. Farrelly, Phys. Rev. A 47, 3113 (1993).

[7] J. von Milczewski and T. Uzer, Phys. Rev. A 56, 220 (1997).

[8] E. A. Solove'v, Sov. Phys. JETP 58, 63 (1983).

[9] D. R. Herrick, Phys. Rev. A 26, 323 (1982).

[10] P. A. Braun, Rev. Mod. Phys. 65, 115 (1993).

[11] R. H. Cushman and D. A. Sadovskií, Physica D 142, 166 (2000).

[12] R. H. Cushman and D. A. Sadovskií, Europhys. Lett. 47, 1 (1999). 
[13] K. Efstathiou, R. H. Cushman, and D. A. Sadovskií, Physica D 194, 250 (2004).

[14] C. R. Schleif and J. B. Delos, Phys. Rev. A 76, 013404 (2007).

[15] K. Efstathiou, D. A. Sadovskií, and B. I. Zhilinskií, Precis. Eng. 463, 1771 (2007).

[16] J. B. Delos, C. R. Schleif, and G. Dhont, J. Phys.: Conf. Ser. 99, 012005 (2008).

[17] J. J. Duistermaat, Commun. Pure Appl. Math. 33, 687 (1980); R. Cushman and L. Bates, Global Aspects of Classical Integrable Systems (Birkhauser, Boston, 1997); M. S. Child, J. Phys. A 31, 657 (1988); S. V. Ngòc, Commun. Math. Phys. 203, 465 (1999); B. I. Zhilinskií, Acta Appl. Math. 87, 281 (2005).

[18] E. L. Stiefel and G. Scheifele, Linear and Regular Celestial Mechanics (Springer-Verlag, New York, 1970).

[19] G. D. Birkhoff, Dynamical Systems (AMS, New York, 1966), Vol. IX; F. G. Gustavson, Astron. J. 71, 670 (1966).

[20] M. L. Du and J. B. Delos, Phys. Rev. A 38, 1896 (1988); 38, 1913 (1988); J. Gao, J. B. Delos, and M. Baruch, ibid. 46, 1449 (1992); J. Gao and J. B. Delos, ibid. 46, 1455 (1992); 49, 869 (1994); J. Main and G. Wunner, J. Phys. B 27, 2835 (1994); D. M. Wang and J. B. Delos, Phys. Rev. A 63, 043409 (2001); S. Freund, R. Ubert, E. Flöthmann, K. Welge, D. M. Wang, and J. B. Delos, ibid. 65, 053408 (2002); T. Bartsch, J. Main, and G. Wunner, ibid. 67, 063410 (2003); 67, 063411 (2003).

[21] Nearly perpendicular weak fields means $|\chi| \lesssim \omega_{f} N^{3} \ll 1$.

[22] M. Born, The Mechanics of the Atom (Frederick Ungar, New York, 1927 and 1960).

[23] H. Goldstein, Classical Mechanics (Addison-Wesley, New York, 2002).

[24] J. B. Delos, S. K. Knudson, and D. W. Noid, Phys. Rev. A 28, 7 (1983).

[25] L. I. Schiff, Quantum Mechanics (McGraw-Hill, London, 1955 and 1968).

[26] This 2-form is undefined at points where $\left|\mu_{i}\right|= \pm N / 2$, but all necessary integrals are well defined.

[27] These circles have coordinates $\psi_{1}$ or $\psi_{2}$, whichever survives according to the discussion in Sec. III B.

[28] The space of Pauli orbits at fixed $Q$ [the $\left(W, \delta_{W}\right)$ surface] is homeomorphic to a sphere, but we do not establish that it is diffeomorphic to a sphere.

[29] The classical spectrum is also known as the image of the energy momentum map.

[30] For some parameters, a dashed red curve appears on classically doubly degenerate points of the classical spectrum. Specific examples are the upper boundary of the inner triangular region for the Stark region, and the lower boundary of the inner triangular region for the Zeeman region. In these cases, only one of the two disjoint components of the level set is not a 2-torus. Such cases will always be evident when inspecting the contours of $h_{2}$ on the $\left(W, \delta_{W}\right)$ surface.

[31] Equations (40) cannot be linearized at the poles of the $\left(W, \delta_{W}\right)$ surface, and extrema at the poles are not quadratic.

[32] V. I. Arnold, Mathematical Methods of Classical Mechanics (Springer-Verlag, New York, 1979).

[33] The reference point of the action may be taken to be at $Q=0$. All other choices of reference point would yield an action that differs from Eq. (43) by an additive constant.

[34] For $0<|Q|<N$, close examination of curves passing through the poles is needed.

[35] By construction, the action is defined only for areas bounded by contours of $h_{2}\left(W, \delta_{W}\right)$ which do not contain fixed points. See Chap. 10 of Ref. [32].

[36] E. U. Condon and G. H. Shortley, The Theory of Atomic Spectra (Cambridge University Press, New York, 1935).

[37] The quantum basis must include several $n$-manifolds or important effects, second order in the electric field, will be neglected.

[38] In the definitions of the $\hat{Q}$ and $\hat{W}$ operators, the field vectors $\mathbf{F}$ and $\mathbf{B}$ can be replaced with arbitrary vectors $\mathbf{V}_{1}$ and $\mathbf{V}_{2}$ such that the operators $\hat{H}_{0}, \hat{Q}$, and $\hat{W}$ will form a complete set of commuting observables on a basis of bound states of the unperturbed hydrogen atom.

[39] A lattice can be constructed from a list of energy levels alone. See Ref. [14].

[40] In the Stark limit, the equator $W=0$ has exceptional structure. It is composed entirely of relative fixed points. Similar exceptional structure is found on $\Gamma_{N, Q=0}$ for $\mathbf{F}$ and $\mathbf{B}$ that lie on the (black and blue online) curves dividing regions of the map.

[41] Quantum calculations also show that states belonging to different $q$ - and $n$-manifolds also exhibit anticrossings with variations in $\omega_{f}$.

[42] See EPAPS Document No. E-PLRAAN-77-087804 for a detailed derivation. For more information on EPAPS, see http:// www.aip.org/pubservs/epaps.html. This material is also available for download from www.chrisschleif.com

[43] See Chap. 22 of Ref. [22] or Chap. 3.8 of Ref. [23].

[44] See Fig. 13 of Ref. [22].

[45] See Chap. 4.4 of Ref. [23].

[46] In the six-dimensional phase space $\mathbb{R}^{2 n}$, either $K$ is diffeomorphic to a circle $(L \neq 0)$ or its compactification is. Each point $x \in K \subset \mathbb{R}^{2 n}$ is labeled by a value of the Delaunay coordinate $\phi_{N}$. 